Supporting information for:

\title{
Wrapping Rhodium in a Borane Canopy: Implications for Hydride Formation and Transfer
}

Joseph A. Zurakowski, Brady J. H. Austen, and Marcus W. Drover*

\begin{abstract}
*E-mail: marcus.drover@uwindsor.ca
aDepartment of Chemistry and Biochemistry, The University of Windsor, 401 Sunset Avenue, Windsor, ON, N9B 3P4, Canada
\end{abstract}

1. Summary Table S2

2. Multinuclear NMR data

S3

3. Crystallography

S34

4. Computational Chemistry

S37 
Table S1. Summary of Data obtained for Rhodium Compounds

\begin{tabular}{|c|c|c|c|c|c|}
\hline Compound & Name & Formula & $\mathrm{Mw}(\mathrm{g} / \mathrm{mol})$ & $\delta$ р (ppm) & ${ }^{1} J_{\mathrm{Rh}, \mathrm{P}}(\mathrm{Hz})$ \\
\hline$[1] \mathrm{BPh}_{4}$ & $\left\{\left[\mathrm{Rh}(\text { tape })_{2}\right] \mathrm{BPh}_{4}\right\}$ & $\mathrm{C}_{52} \mathrm{H}_{68} \mathrm{BP} \mathrm{P}_{4} \mathrm{Rh}$ & 930.7 & 46.2 & 126.8 \\
\hline$[2] \mathrm{BPh}_{4}$ & $\left\{\left[\mathrm{Rh}(\mathrm{d} n \mathrm{ppe})_{2}\right] \mathrm{BPh}{ }_{4}\right\}$ & $\mathrm{C}_{52} \mathrm{H}_{84} \mathrm{BP} \mathrm{P}_{4} \mathrm{Rh}$ & 946.5 & 53.7 & 125.5 \\
\hline$[3] \mathrm{BPh}_{4}$ & $\left.\left\{\left[\mathrm{Rh}\left(\mathrm{P}_{2} \mathrm{~B}^{\mathrm{Cy}}\right)_{4}\right)_{2}\right] \mathrm{BPh}_{4}\right\}$ & $\mathrm{C}_{148} \mathrm{H}_{252} \mathrm{~B}_{9} \mathrm{P}_{4} \mathrm{Rh}$ & 2355.7 & 54.1 & 125.1 \\
\hline$[4]^{7-}$ & {$\left[\mathrm{Rh}\left(\mathrm{P}_{2} \mathrm{~B}^{\mathrm{Cy}} \mathrm{y}_{4}\left({ }^{n} \mathrm{Bu}\right)_{4}\right)_{2}\right]^{7-}$} & -- & -- & 46.1 & 121.2 \\
\hline 5 & {$\left[\mathrm{Rh}(\mathrm{d} n \text { ppe })_{2}(\mathrm{H})\right]$} & $\mathrm{C}_{28} \mathrm{H}_{65} \mathrm{BP}_{4} \mathrm{Rh}$ & 628.6 & 48.3 & 139.9 \\
\hline$[6]^{7-}$ & $\begin{array}{l}{\left[\mathrm{Rh}\left(\mathrm{P}_{2} \mathrm{~B}^{\mathrm{Cy}_{4}}(\mathrm{HBEt})_{4}\right)_{2}\right]^{7-}} \\
{\left[\mathrm{Rh}^{\mathrm{I}}\left(\mathrm{P}_{2} \mathrm{~B}^{\mathrm{C} \mathrm{y}_{4}}-\mu-\mathrm{B}-\mathrm{H}-\right.\right.}\end{array}$ & -- & -- & 53.1 & 122.2 \\
\hline 7 & B) $\left.\left(\mathrm{P}_{2} \mathrm{~B}^{\mathrm{Cy}_{4}}\right)\right]$ & $\mathrm{C}_{124} \mathrm{H}_{233} \mathrm{~B}_{8} \mathrm{P}_{4} \mathrm{Rh}$ & 2037.5 & 51.4 & N/A \\
\hline 8 & {$\left[\mathrm{Rh}(\mathrm{d} n \mathrm{ppe})_{2}\right]\left[\mathrm{HBCy}_{2}{ }^{n} \mathrm{Oct}\right]$} & $\mathrm{C}_{48} \mathrm{H}_{104} \mathrm{BP} 4 \mathrm{Rh}$ & 919 & 54.0 & 125.3 \\
\hline
\end{tabular}




\section{Multinuclear NMR data:}

Figure S1. [1] BPh ${ }_{4}{ }^{1} \mathrm{H}$ NMR, THF-d $8,500 \mathrm{MHz}, 298 \mathrm{~K}$.

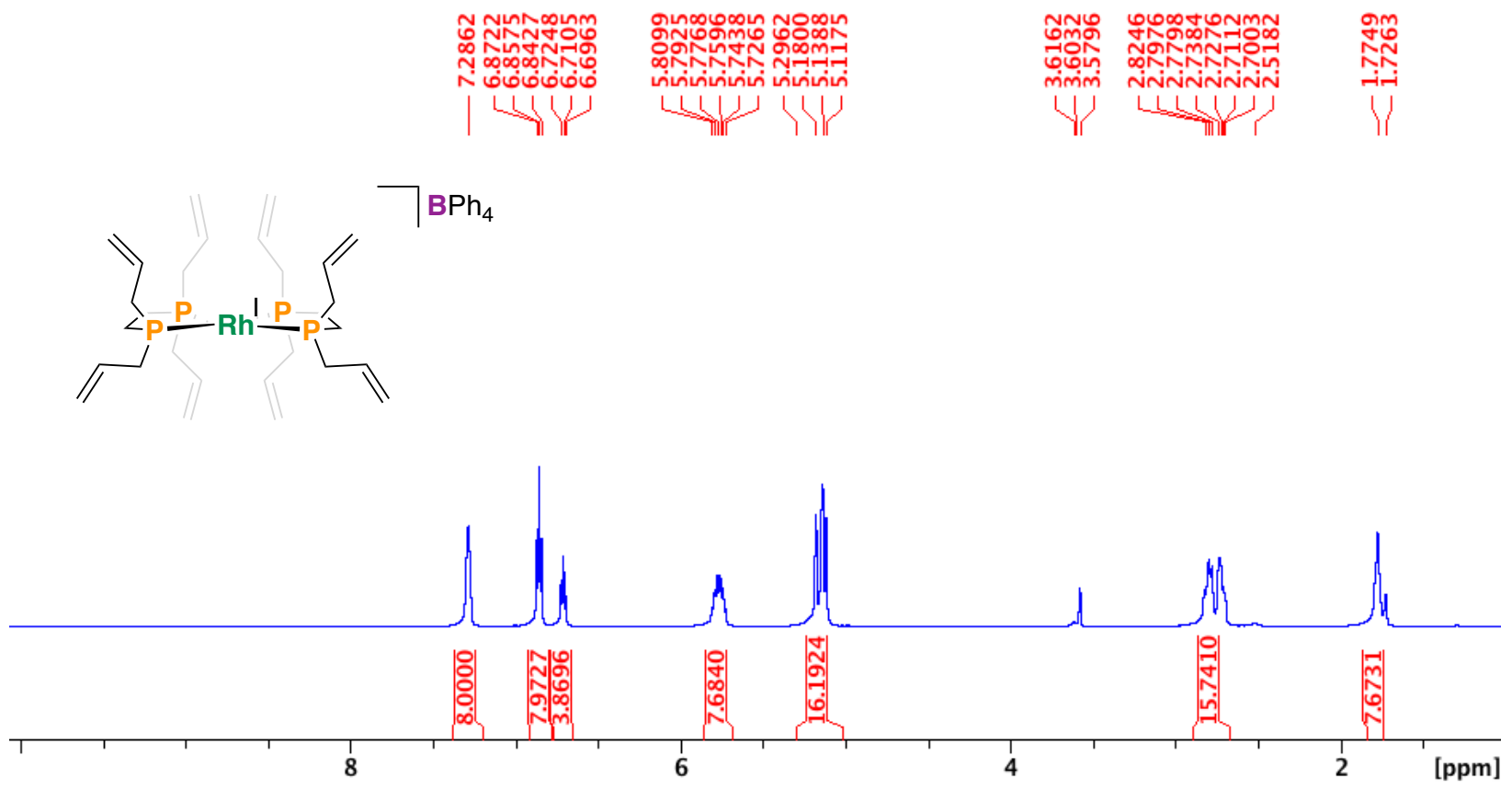

Figure S2. [1]BPh ${ }_{4}$ a) ${ }^{1} \mathrm{H}$ NMR and b) ${ }^{1} \mathrm{H}\left\{{ }^{31} \mathrm{P}\right\}$ NMR, THF- $\mathrm{d}_{8}, 500 \mathrm{MHz}, 298 \mathrm{~K}$.
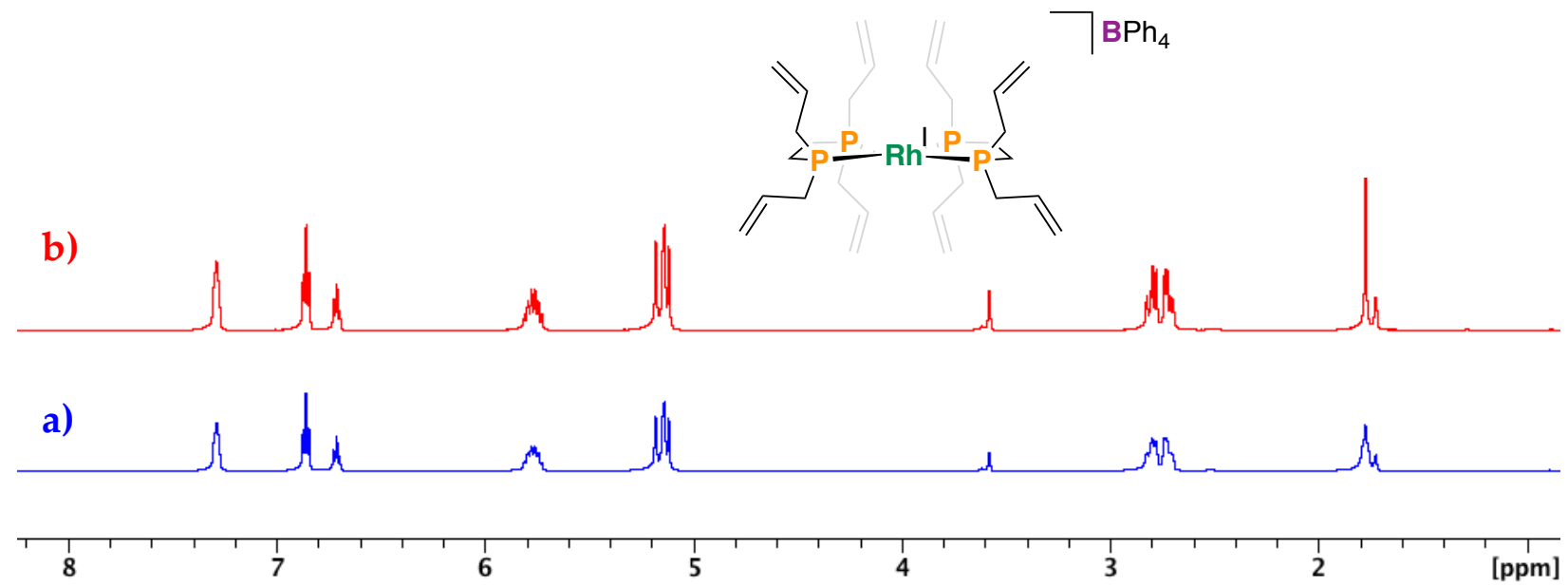
Figure S3. [1]BPh ${ }_{4}{ }^{31} \mathrm{P}\left\{{ }^{1} \mathrm{H}\right\} \mathrm{NMR}, \mathrm{THF}-\mathrm{d}_{8}, 203 \mathrm{MHz}, 298 \mathrm{~K}$.

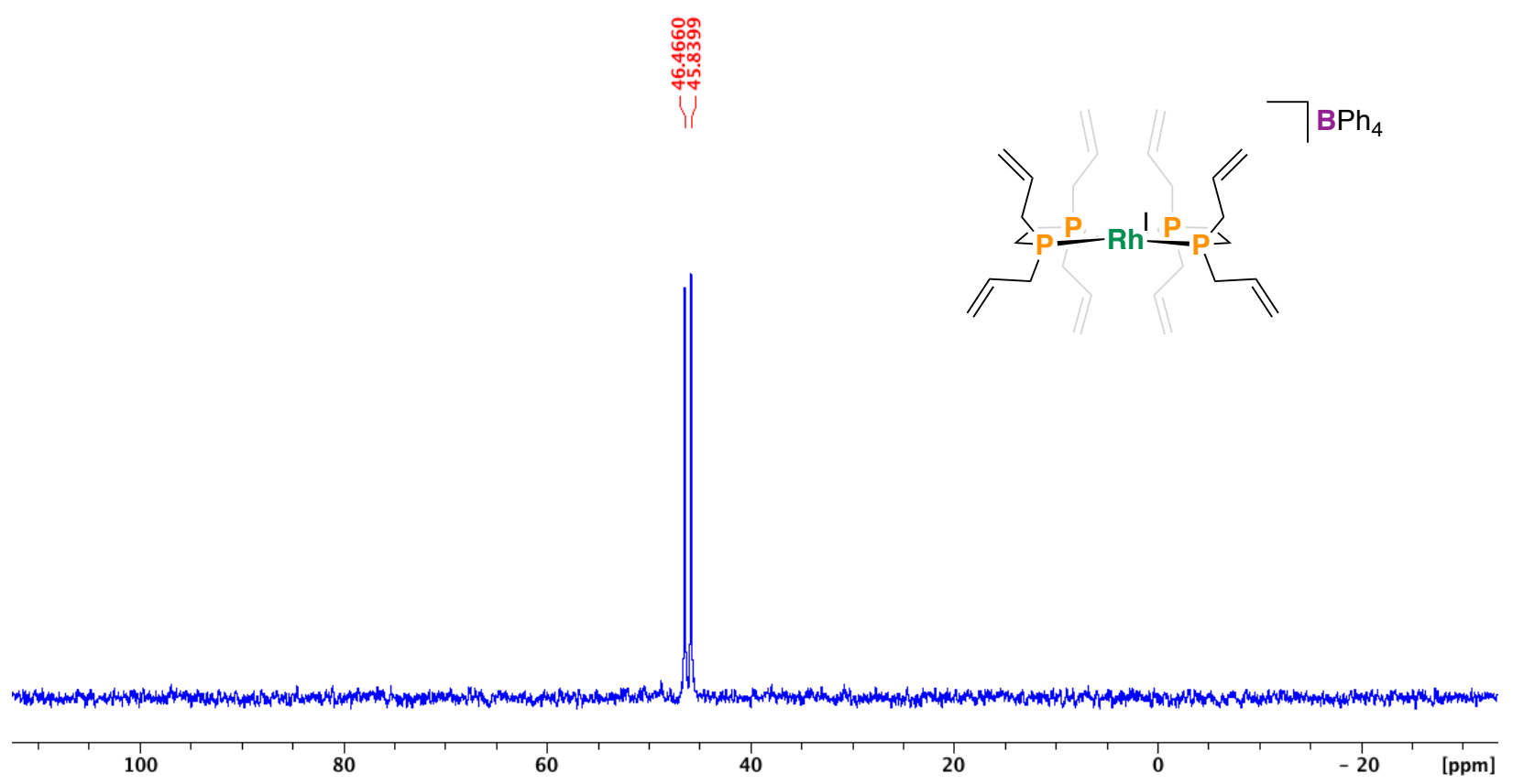

Figure S4. [1]BPh ${ }_{4}{ }^{11} \mathrm{~B}\left\{{ }^{1} \mathrm{H}\right\}$ NMR, THF- ${ }_{8}, 161 \mathrm{MHz}, 298 \mathrm{~K}$.

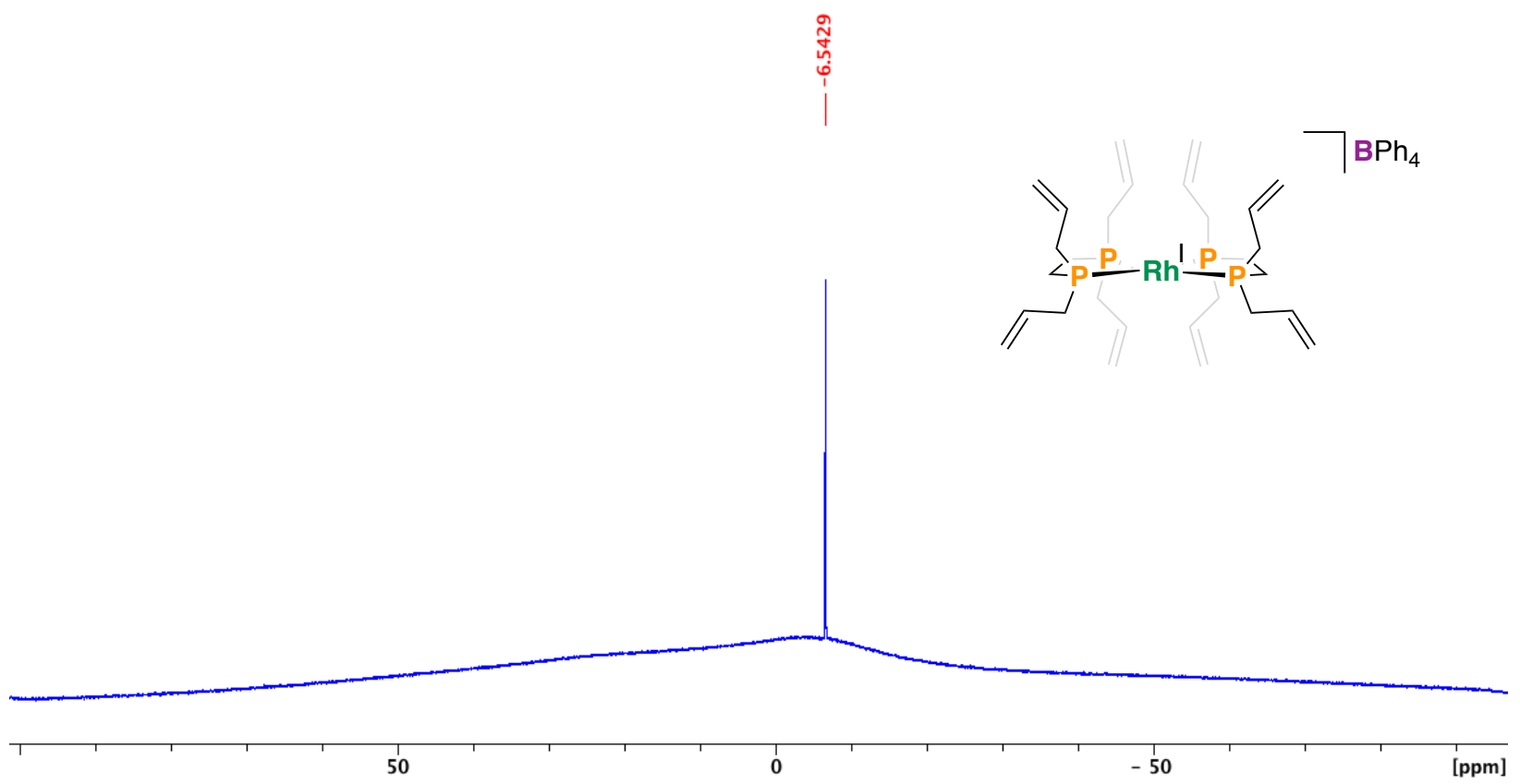


Figure S5. [1]BPh ${ }_{4}{ }^{13} \mathrm{C}\left\{{ }^{1} \mathrm{H}\right\} \mathrm{NMR}, \mathrm{THF}-\mathrm{d}_{8}, 125 \mathrm{MHz}, 298 \mathrm{~K}$.

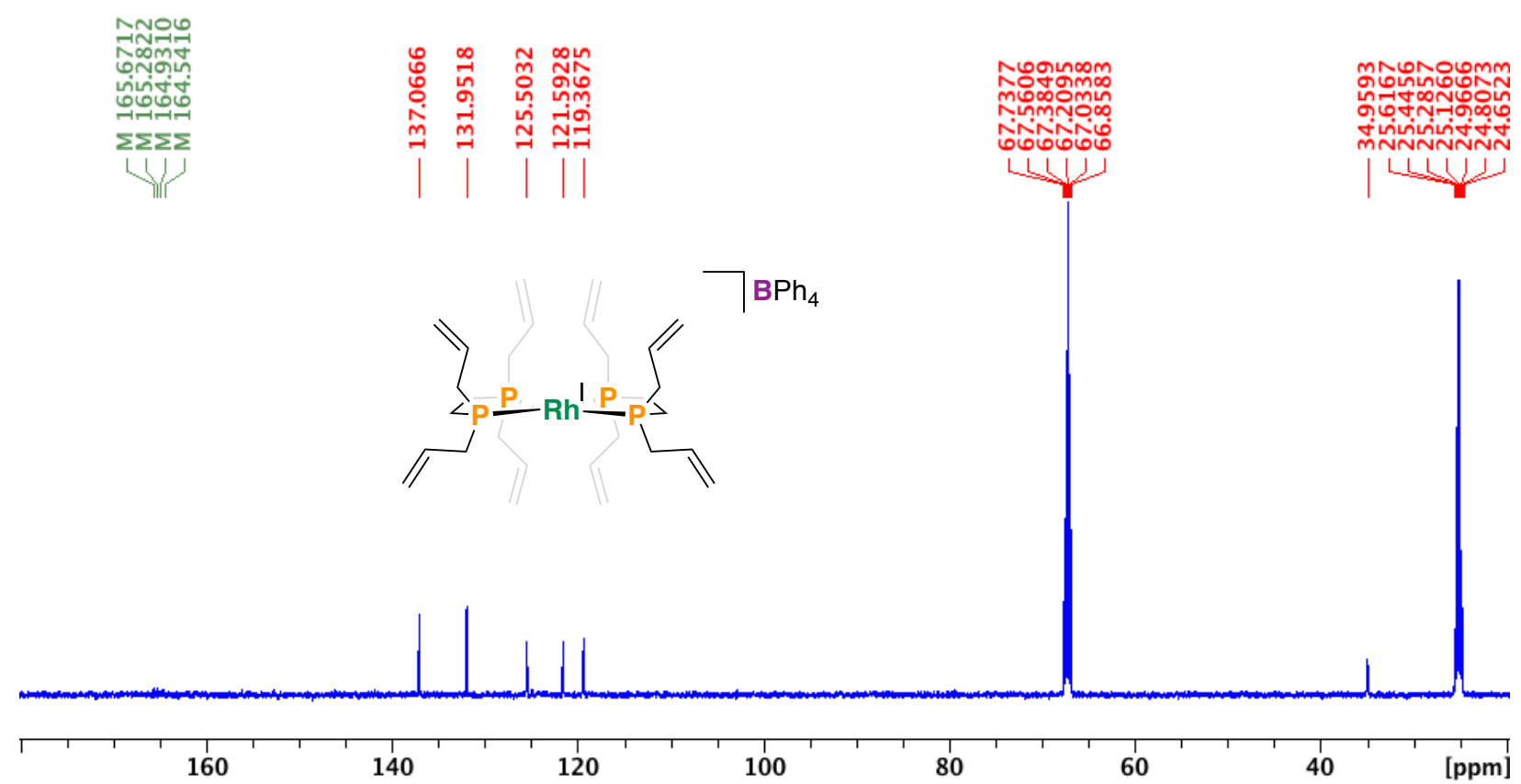

Figure S6. [2]BPh $4,{ }^{1} \mathrm{H}$ NMR, THF- $\mathrm{d}_{8}, 500 \mathrm{MHz}, 298 \mathrm{~K}$.

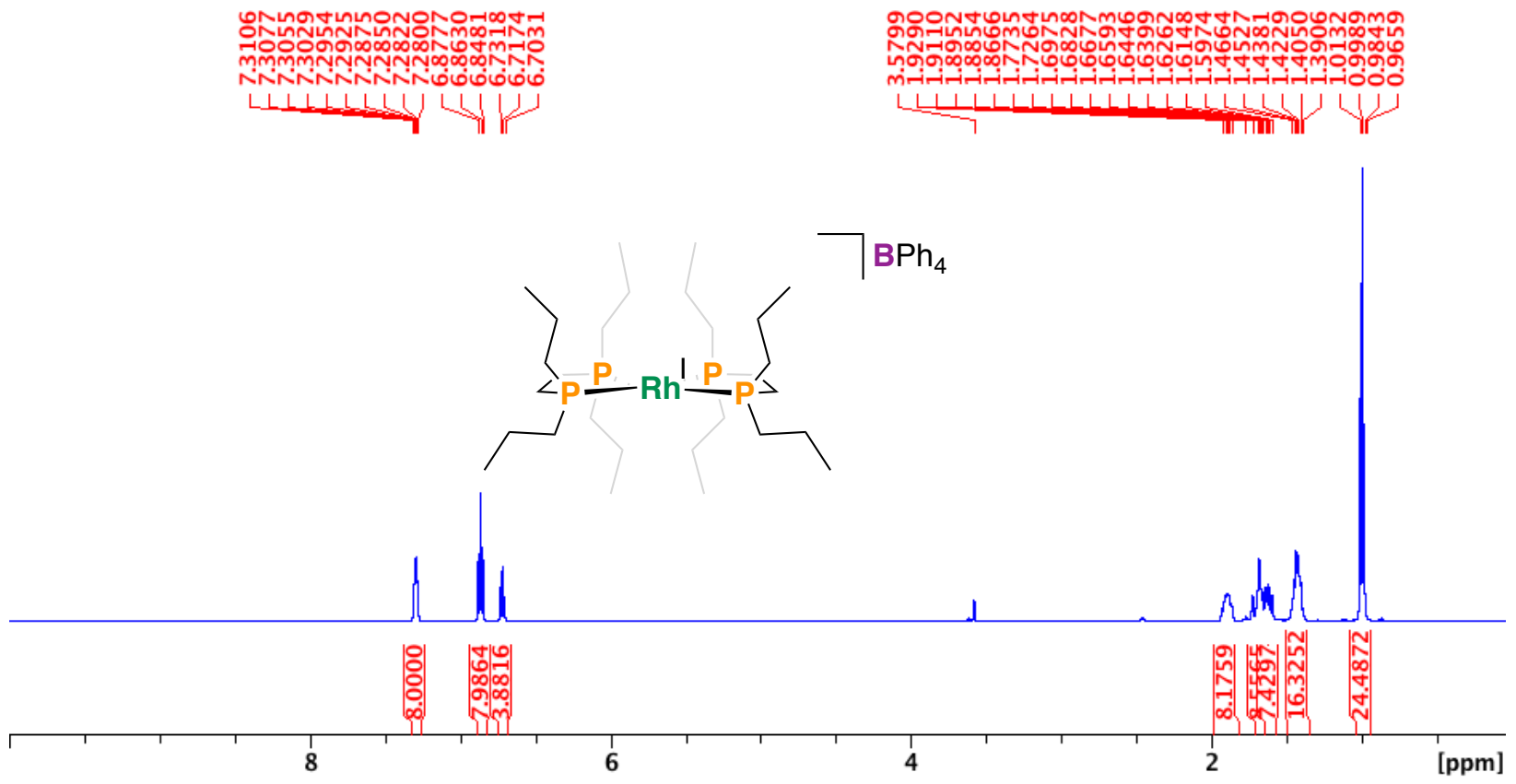


Figure S7. [2]BPh ${ }_{4}{ }^{31} \mathrm{P}\left\{{ }^{1} \mathrm{H}\right\} \mathrm{NMR}, \mathrm{THF}-\mathrm{d}_{8}, 203 \mathrm{MHz}, 298 \mathrm{~K}$.

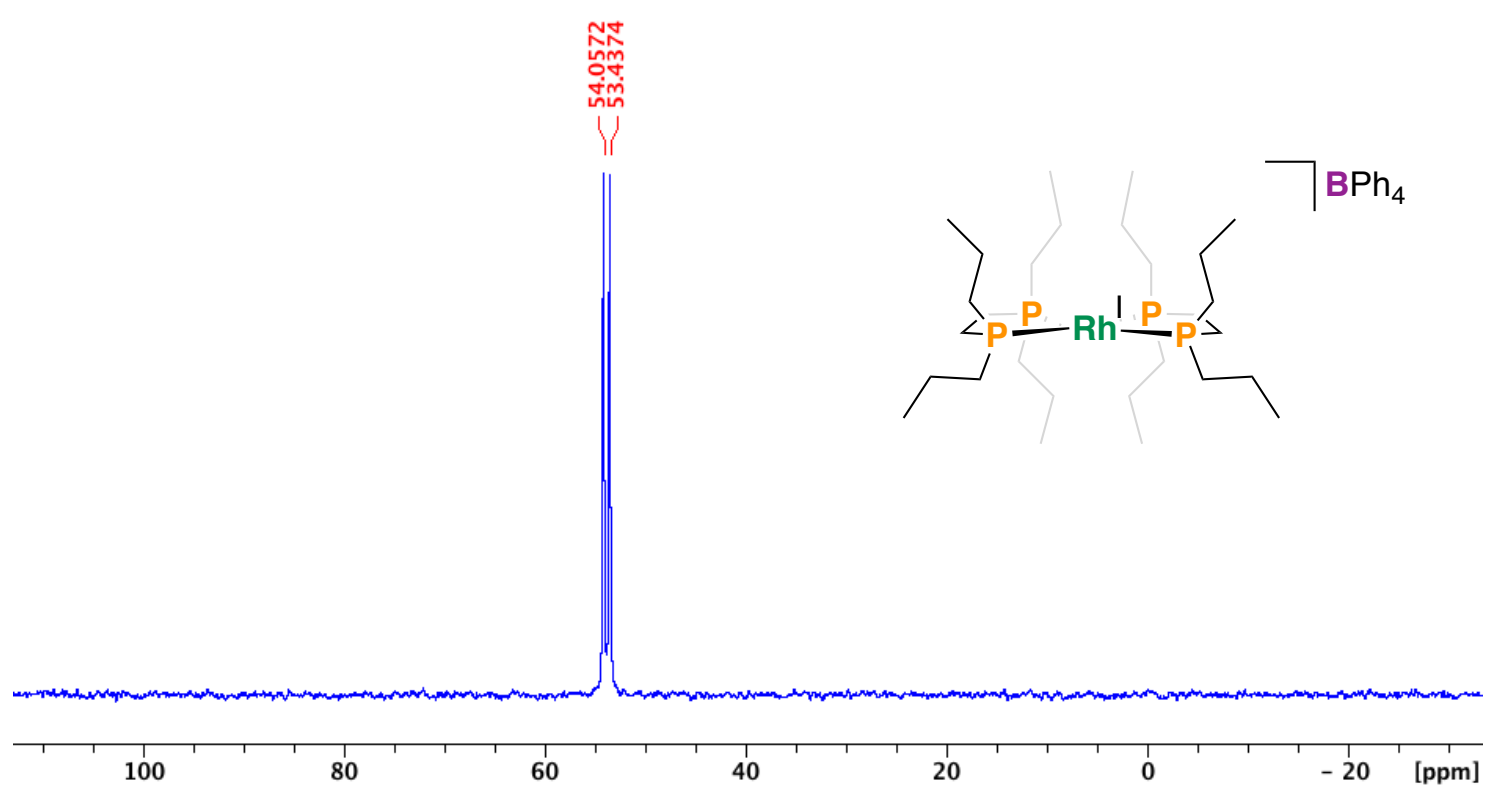

Figure S8. [2]BPh ${ }_{4}{ }^{13} \mathrm{C}\left\{{ }^{1} \mathrm{H}\right\}$ NMR, THF-d $8,125 \mathrm{MHz}, 298 \mathrm{~K}$.

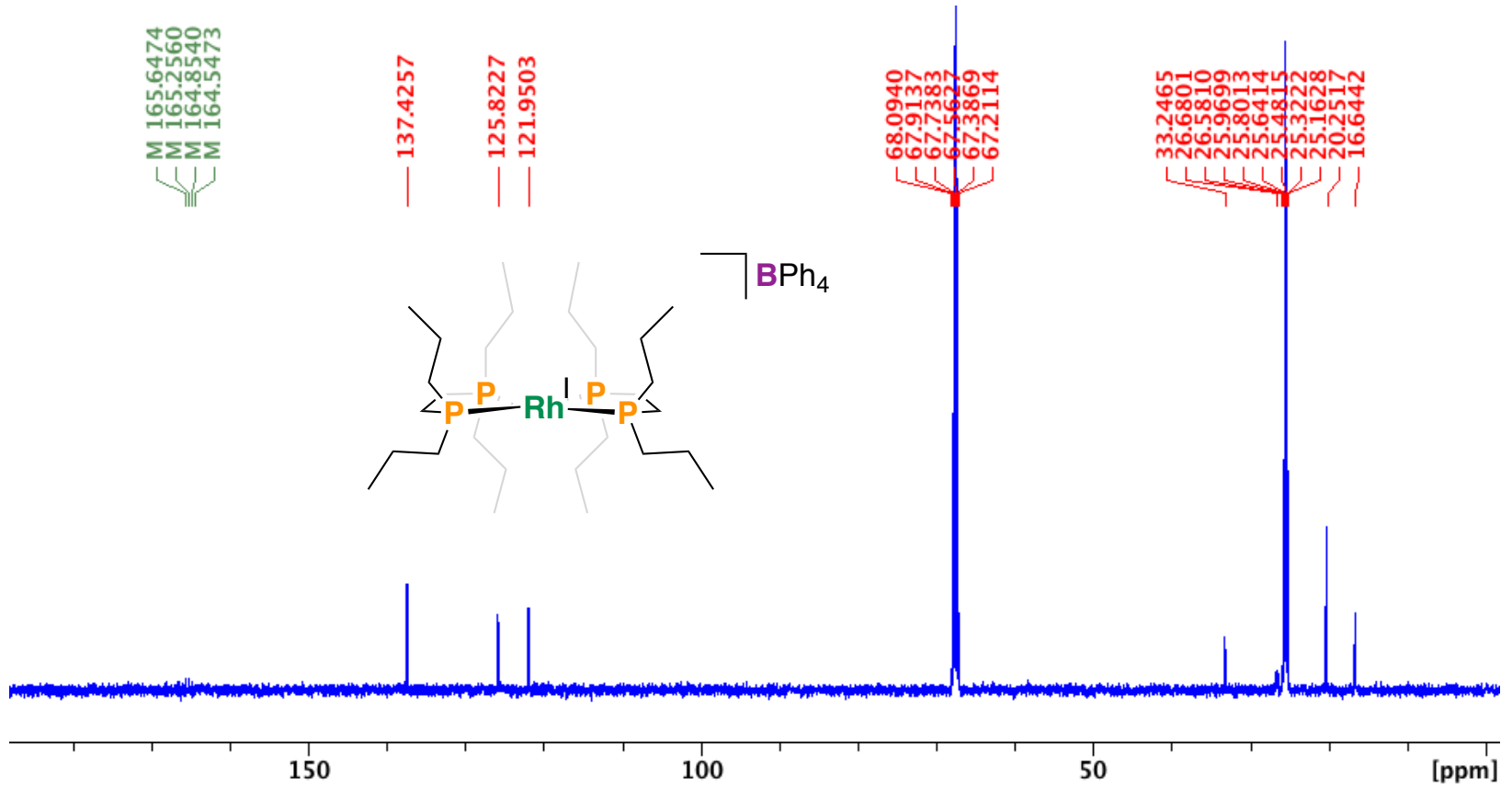


Figure S9. [3] BPh $4,{ }^{1} \mathrm{H}$ NMR, THF-d $\mathrm{d}_{8}, 500 \mathrm{MHz}, 298 \mathrm{~K}$.

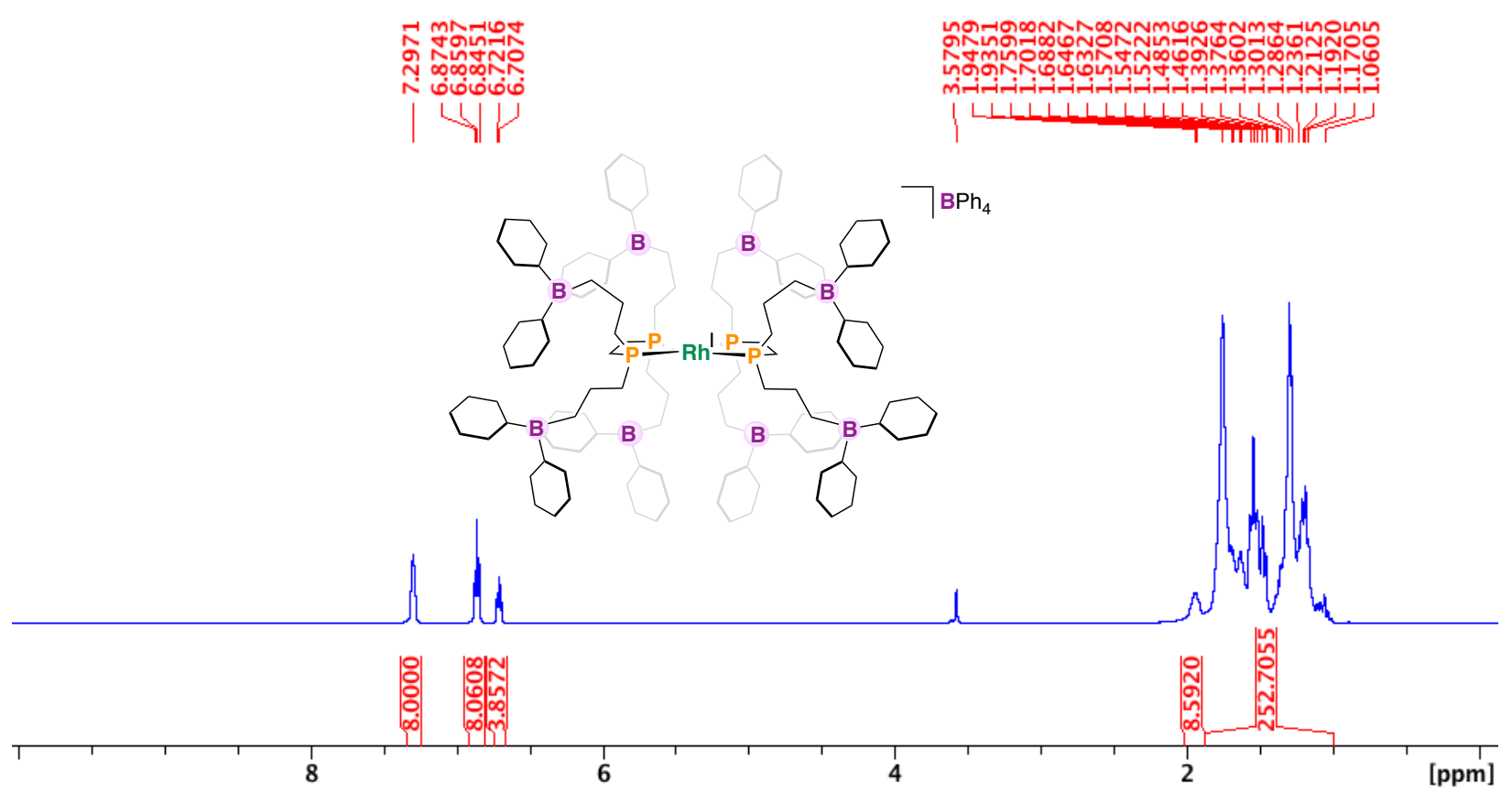

Figure S10. [3] $\mathrm{BPh}$, a) $\left.{ }^{1} \mathrm{H} \mathrm{NMR}, \mathrm{b}\right){ }^{1} \mathrm{H}\left\{{ }^{31} \mathrm{P}\right\} \mathrm{NMR}(\mathrm{o} 2 \mathrm{p}=54 \mathrm{ppm})$, and c) ${ }^{1} \mathrm{H}\left\{{ }^{11} \mathrm{~B}\right\} \mathrm{NMR}$ $(\mathrm{o} 2 \mathrm{p}=84 \mathrm{ppm}), \mathrm{THF}-\mathrm{d}_{8}, 500 \mathrm{MHz}, 298 \mathrm{~K}$.

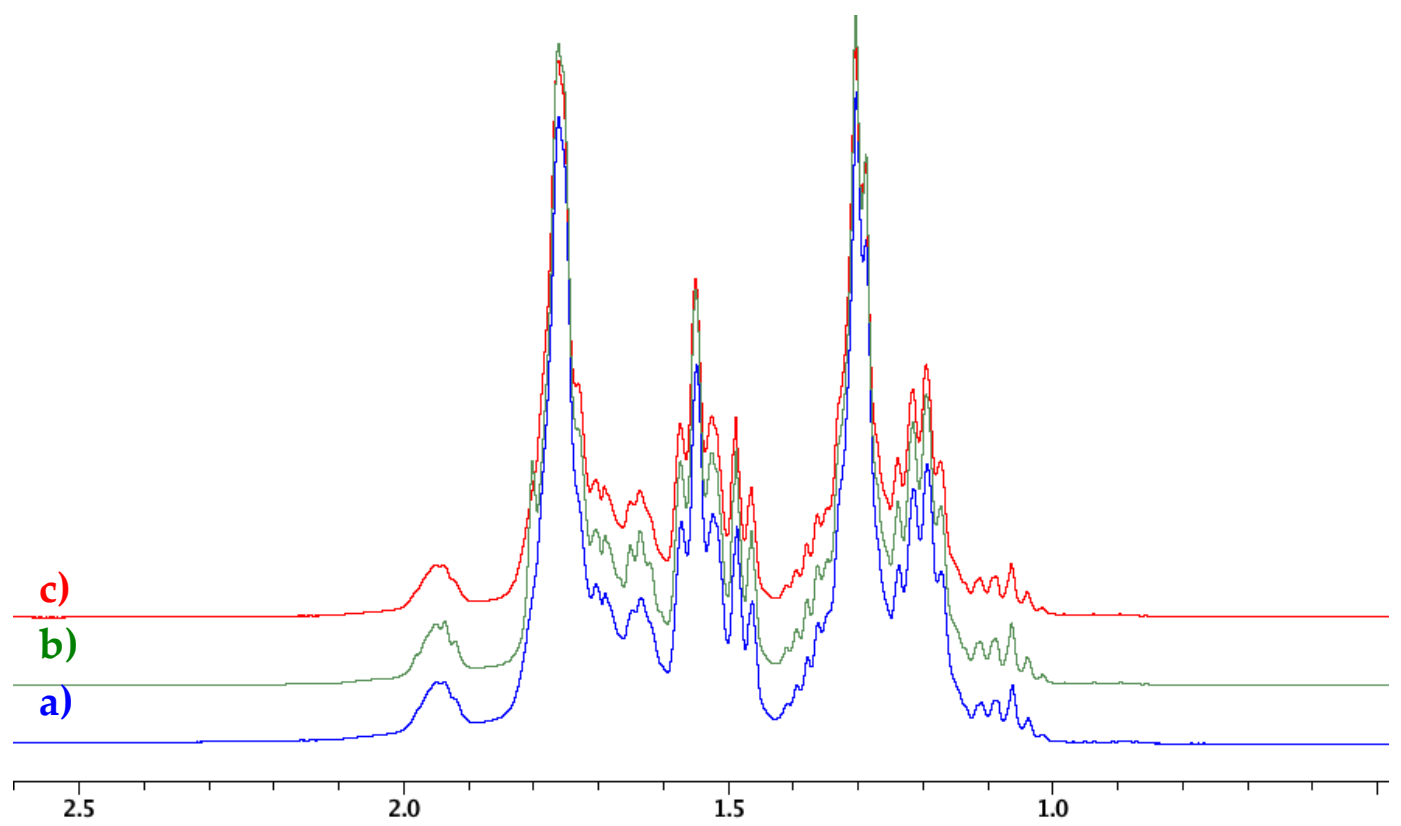


Figure S11. [3] BPh ${ }_{4}{ }^{31} \mathrm{P}\left\{{ }^{1} \mathrm{H}\right\} \mathrm{NMR}, \mathrm{THF}-\mathrm{d}_{8}, 203 \mathrm{MHz}, 298 \mathrm{~K}$.

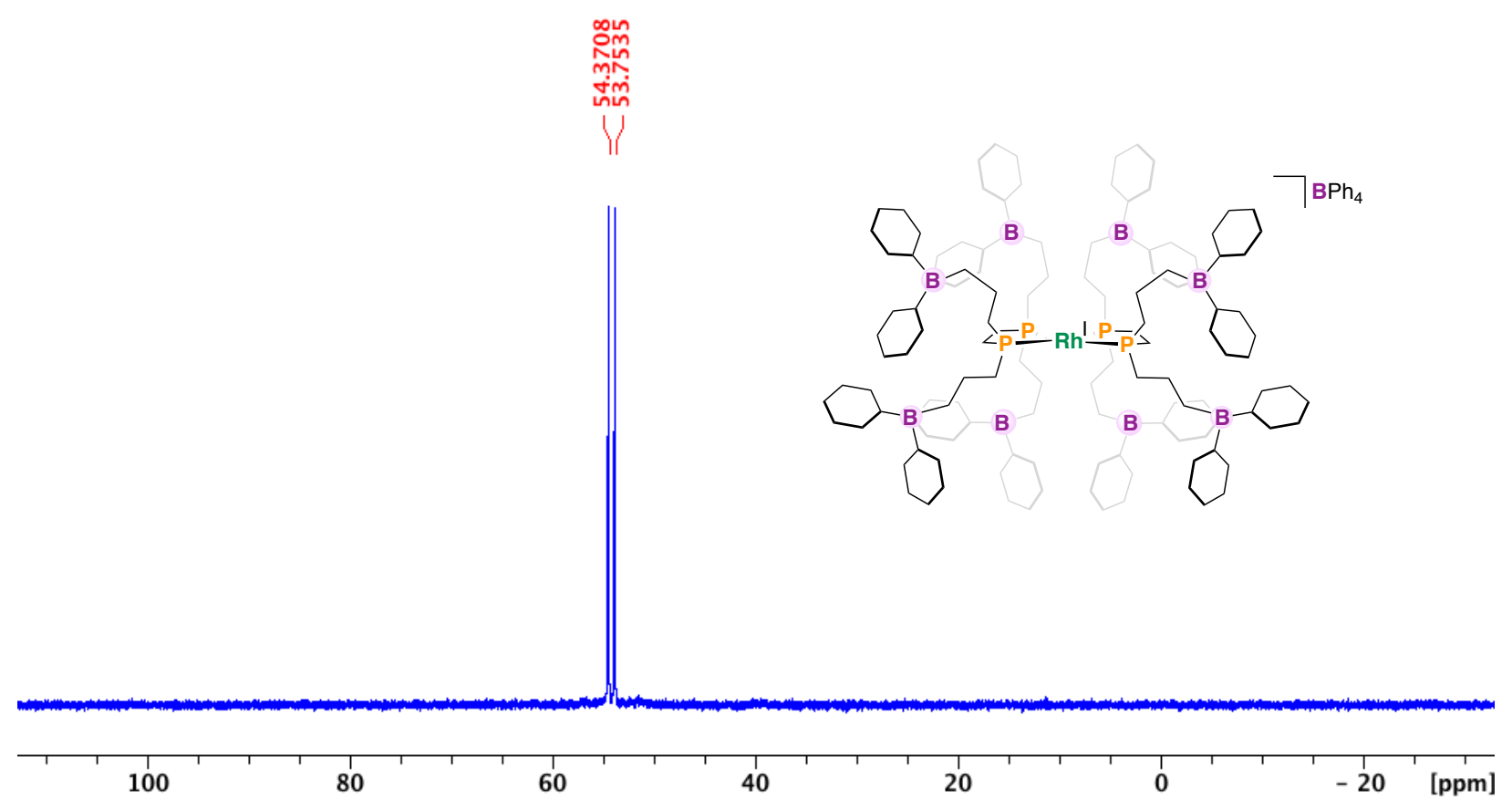

Figure S12. [3] BPh ${ }_{4},{ }^{11} \mathrm{~B}\left\{{ }^{1} \mathrm{H}\right\} \mathrm{NMR}, \mathrm{THF}-\mathrm{d}_{8}, 161 \mathrm{MHz}, 298 \mathrm{~K}$.

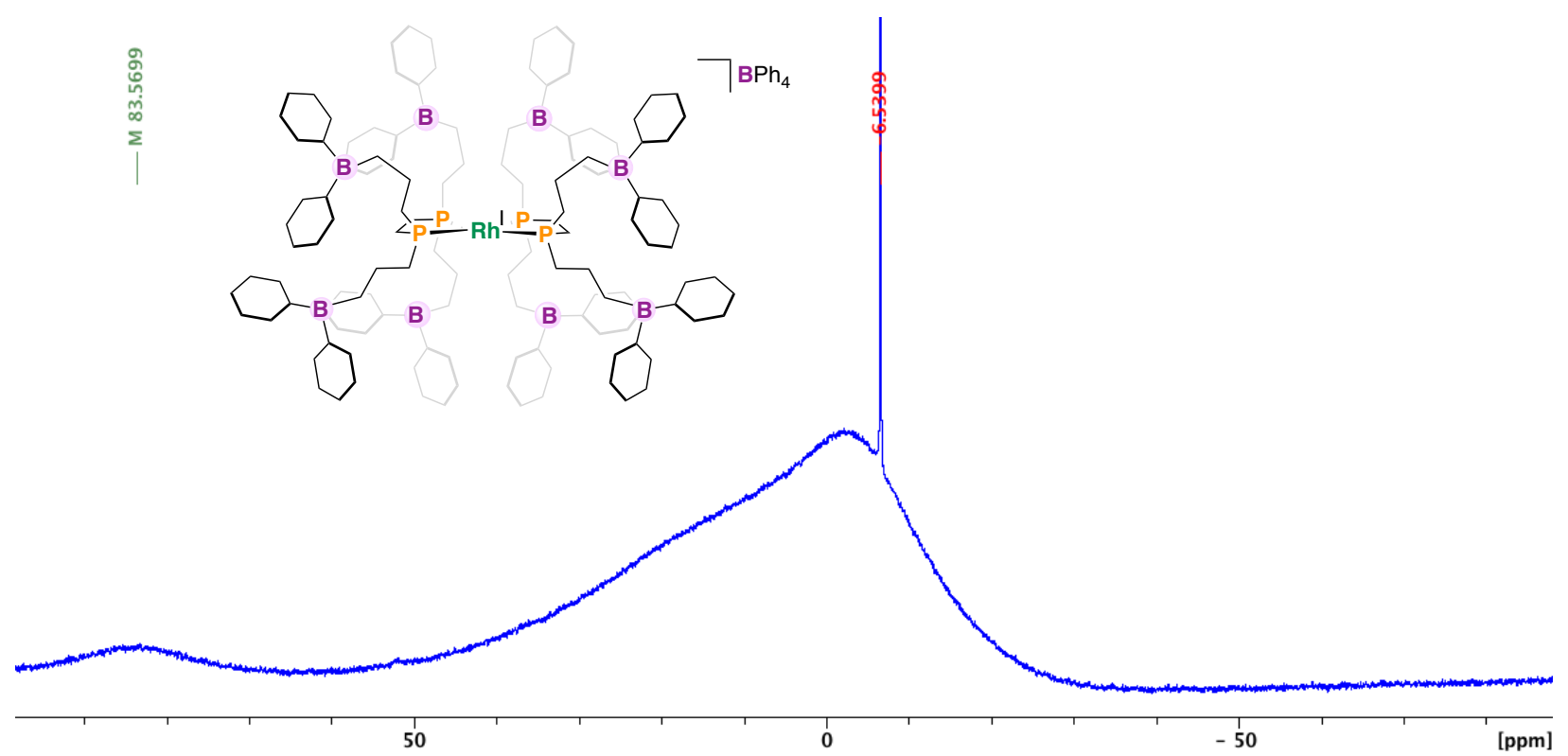


Figure S13. [3] BPh $4,{ }^{13} \mathrm{C}\left\{{ }^{1} \mathrm{H}\right\} \mathrm{NMR}, \mathrm{THF}-\mathrm{d}_{8}, 125 \mathrm{MHz}, 298 \mathrm{~K}$.

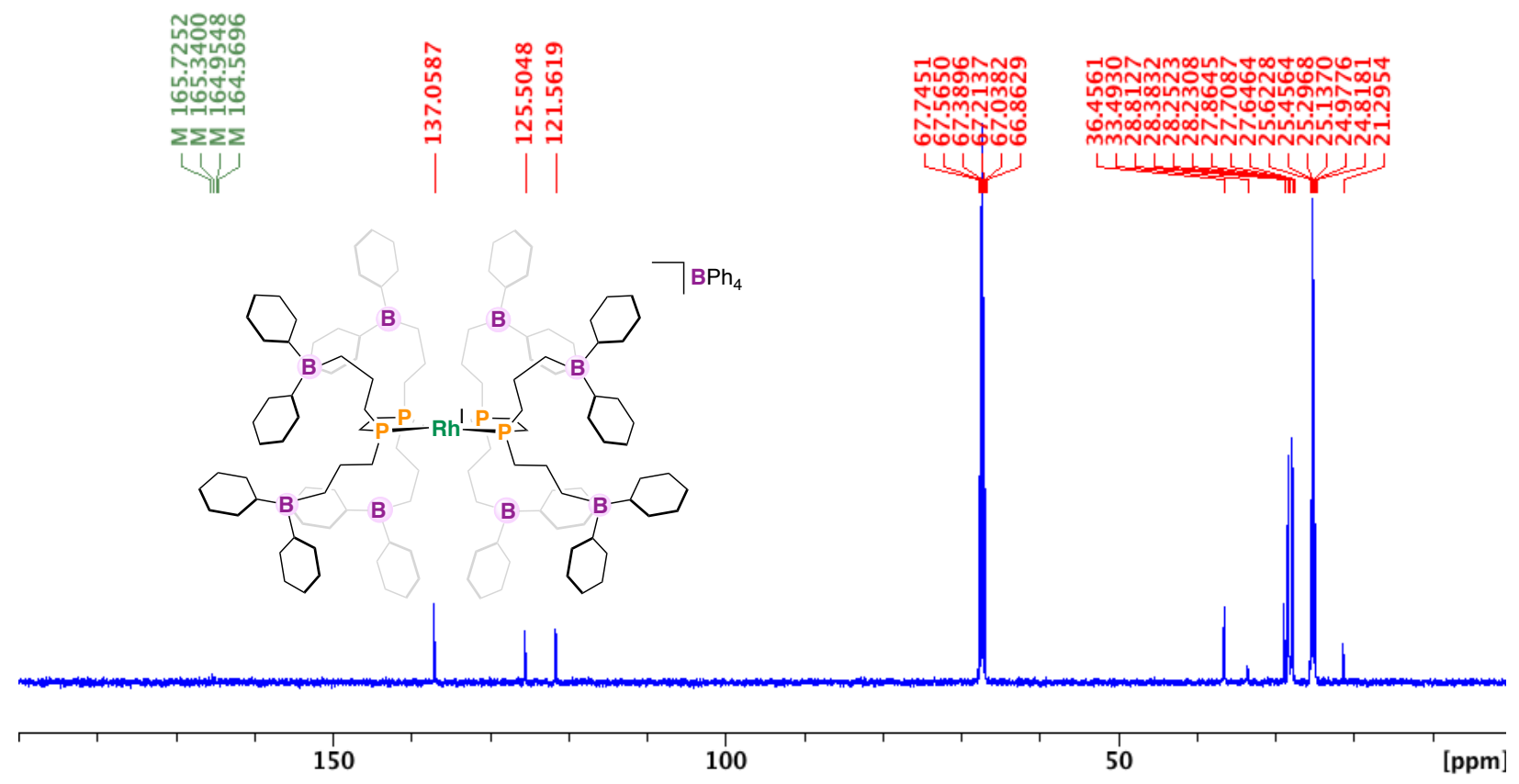

Figure S14. a) $[3]^{+}$and $\mathbf{b}$ ) treatment of $[3]^{+}$with excess (> 50 equivs.) $n$-BuLi, ${ }^{1} \mathrm{H}$ NMR, THF-d $\mathrm{d}_{8}, 500 \mathrm{MHz}, 298 \mathrm{~K}$.

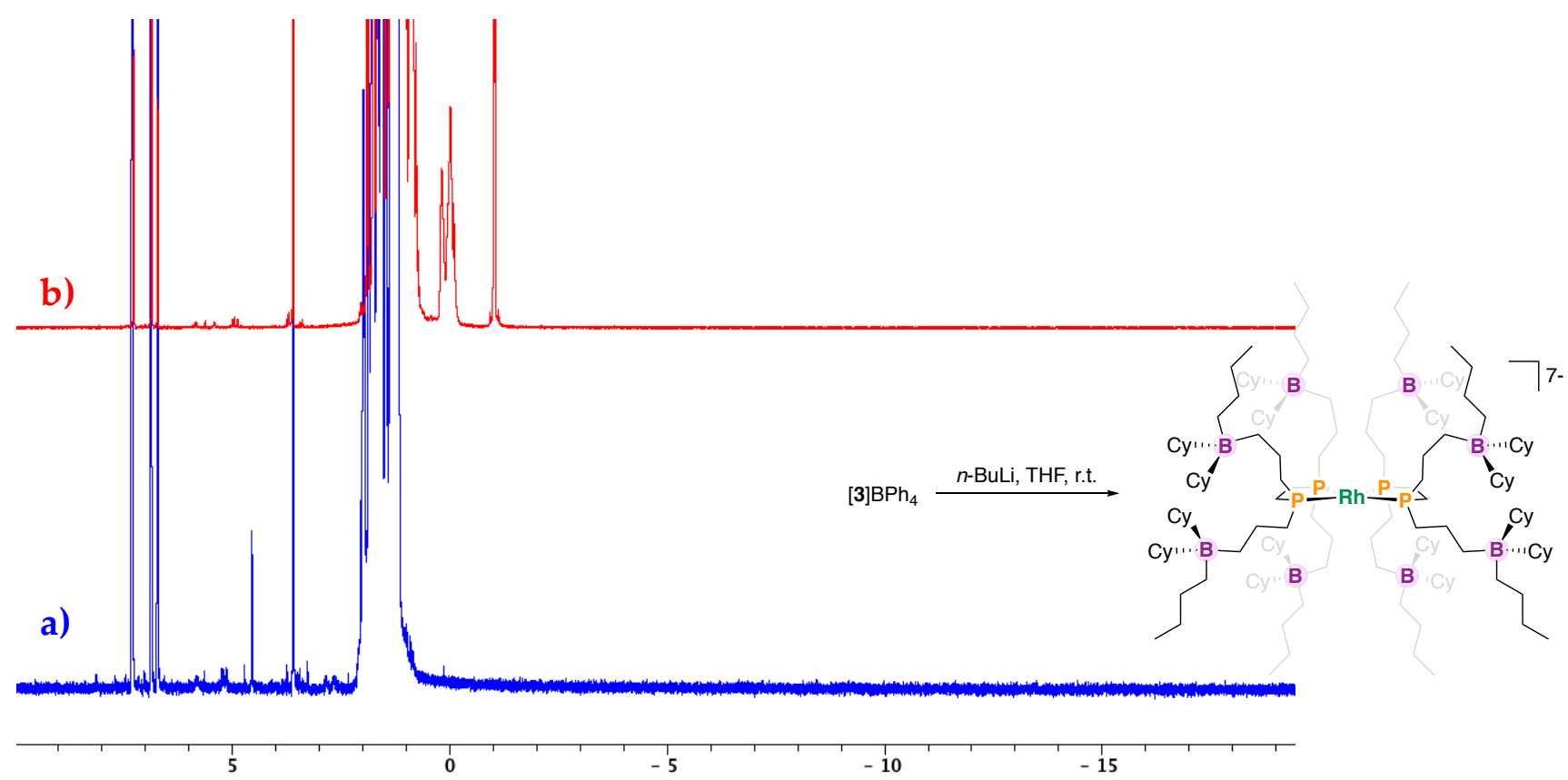


Figure S15. a) $[3]^{+}$and b) treatment of $[3]^{+}$with excess (> 50 equivs.) $n-\mathrm{BuLi},{ }^{1} \mathrm{H}$ NMR, THF-d $8,500 \mathrm{MHz}, 298 \mathrm{~K}$ * $^{*}$ = residual $n$-BuLi

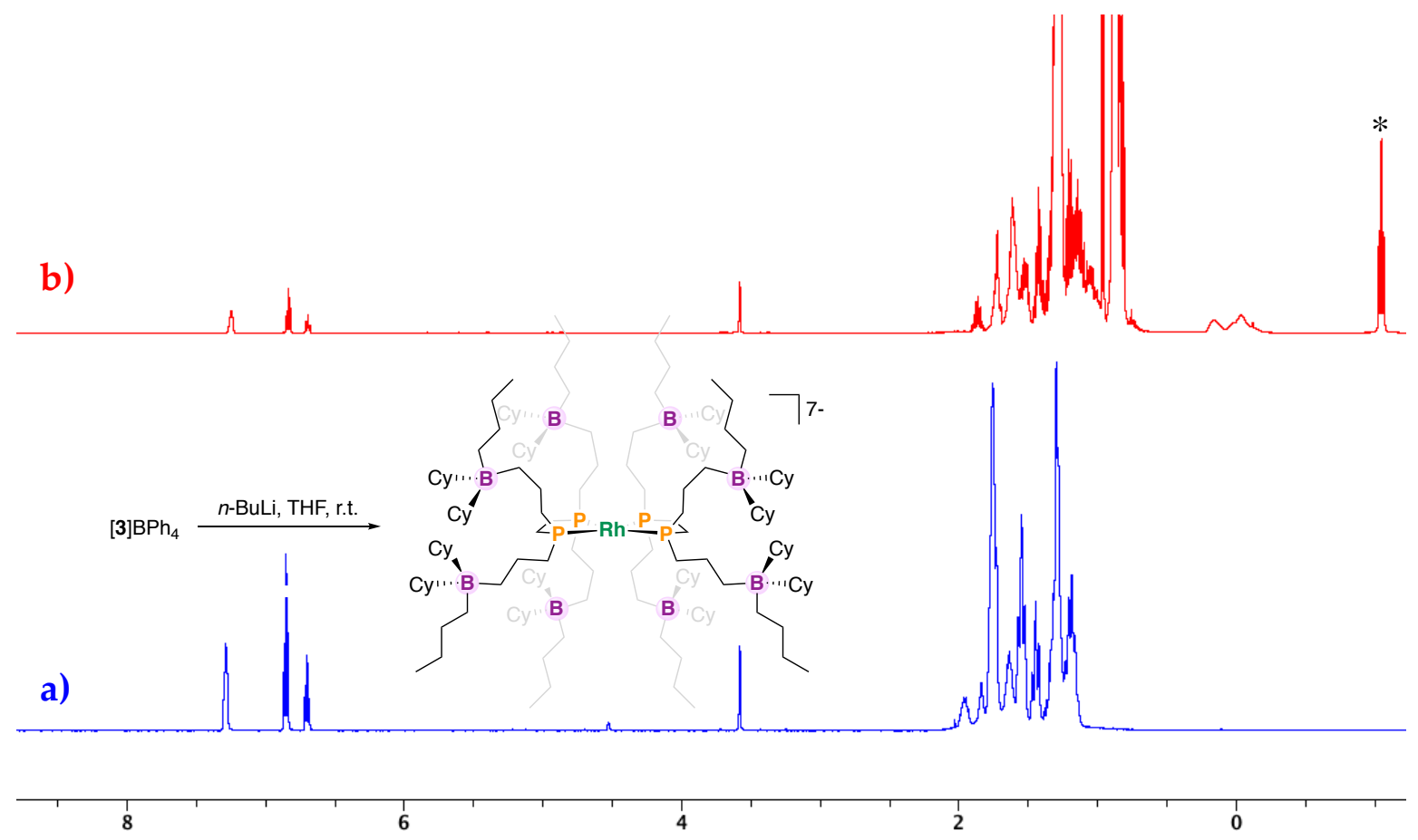

Figure S16. Treatment of [3] $]^{+}$with 8 equivs. $n$-BuLi, ${ }^{1} \mathrm{H}$ NMR, THF-d $\mathrm{d}_{8}, 500 \mathrm{MHz}, 298 \mathrm{~K}$. This spectrum provides $1: 1$ integration with the lost $\mathrm{Li}[\mathrm{BPh} 4]$ counterion $(8: 8: 4)$ and the $\mathrm{CH} / \mathrm{CH}_{2}$ groups $\alpha$ to the borate group $(48 \mathrm{H})$.

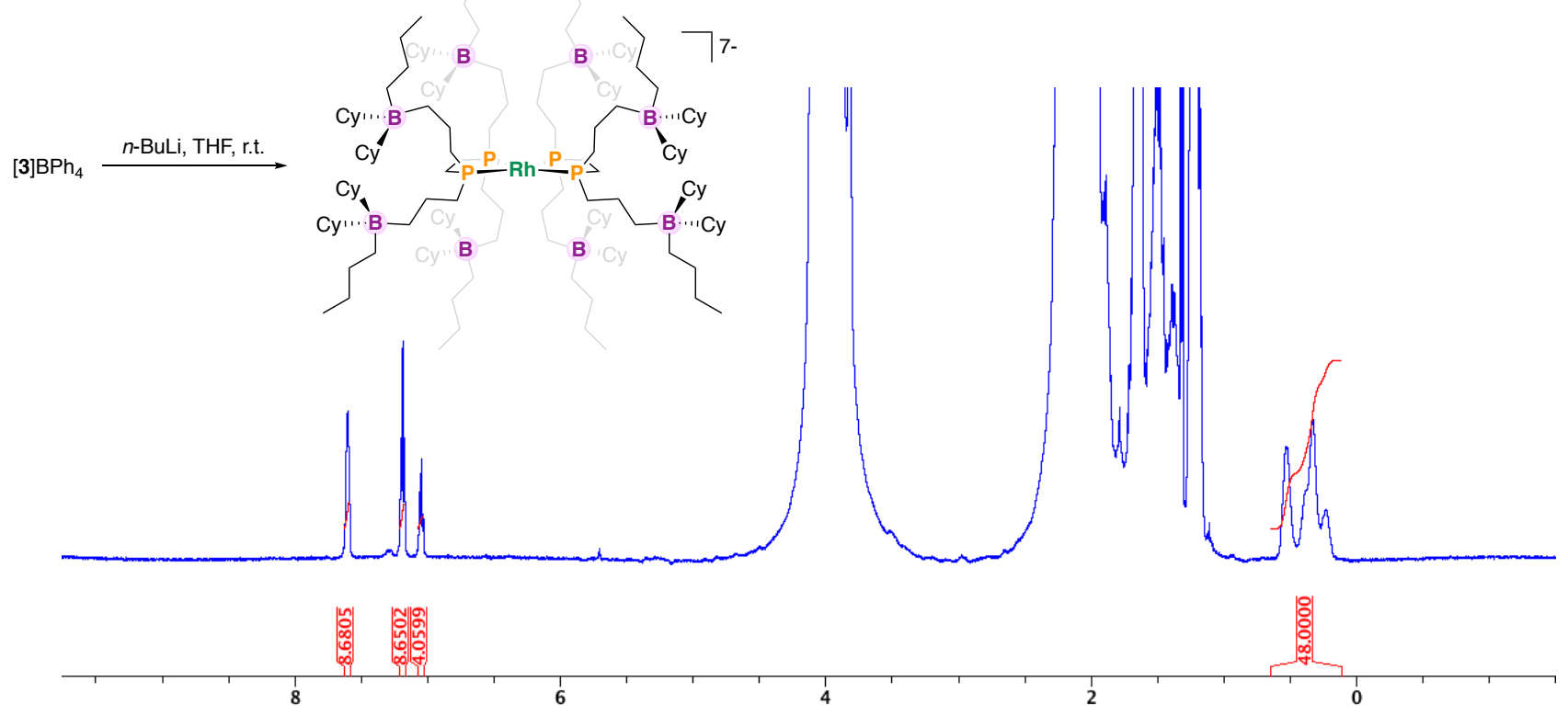


Figure S17. Treatment of [3] ${ }^{+}$with excess (> 50 equivs.) $n$-BuLi, ${ }^{1} \mathrm{H}$ NMR, THF- $\mathrm{d}_{8}, 500$ $\mathrm{MHz}, 298 \mathrm{~K}$. This spectrum provides 1:1 integration with the lost $\mathrm{Li}^{\mathrm{BPh}} 4$ ] counterion (8:8:4) and the $\mathrm{CH} / \mathrm{CH}_{2}$ groups $\alpha$ to the borate group $(48 \mathrm{H})$.

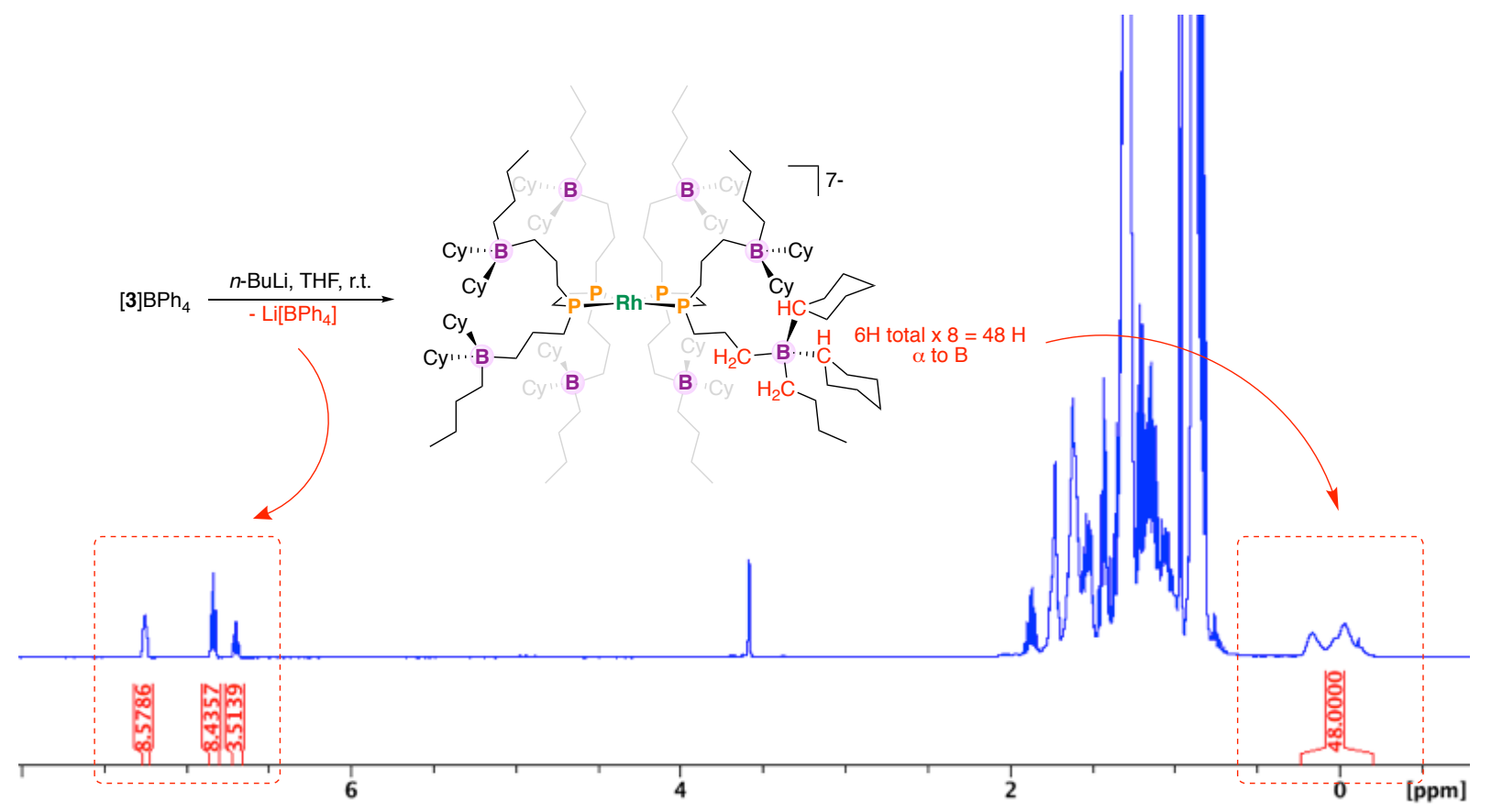

Figure S18. Treatment of [3] $]^{+}$with excess (> 50 equivs.) $n$-BuLi, a) ${ }^{1} \mathrm{H}$ NMR, b) ${ }^{1} \mathrm{H}\left\{{ }^{11} \mathrm{~B}\right\}$ $\mathrm{NMR}$, and c) ${ }^{1} \mathrm{H}\left\{{ }^{31} \mathrm{P}\right\} \mathrm{NMR}, \mathrm{THF}-\mathrm{d}_{8}, 500 \mathrm{MHz}, 298 \mathrm{~K}$. This spectrum shows the $\mathrm{CH} / \mathrm{CH}_{2}$ groups $\alpha$ to the borate group $(48 \mathrm{H})$.
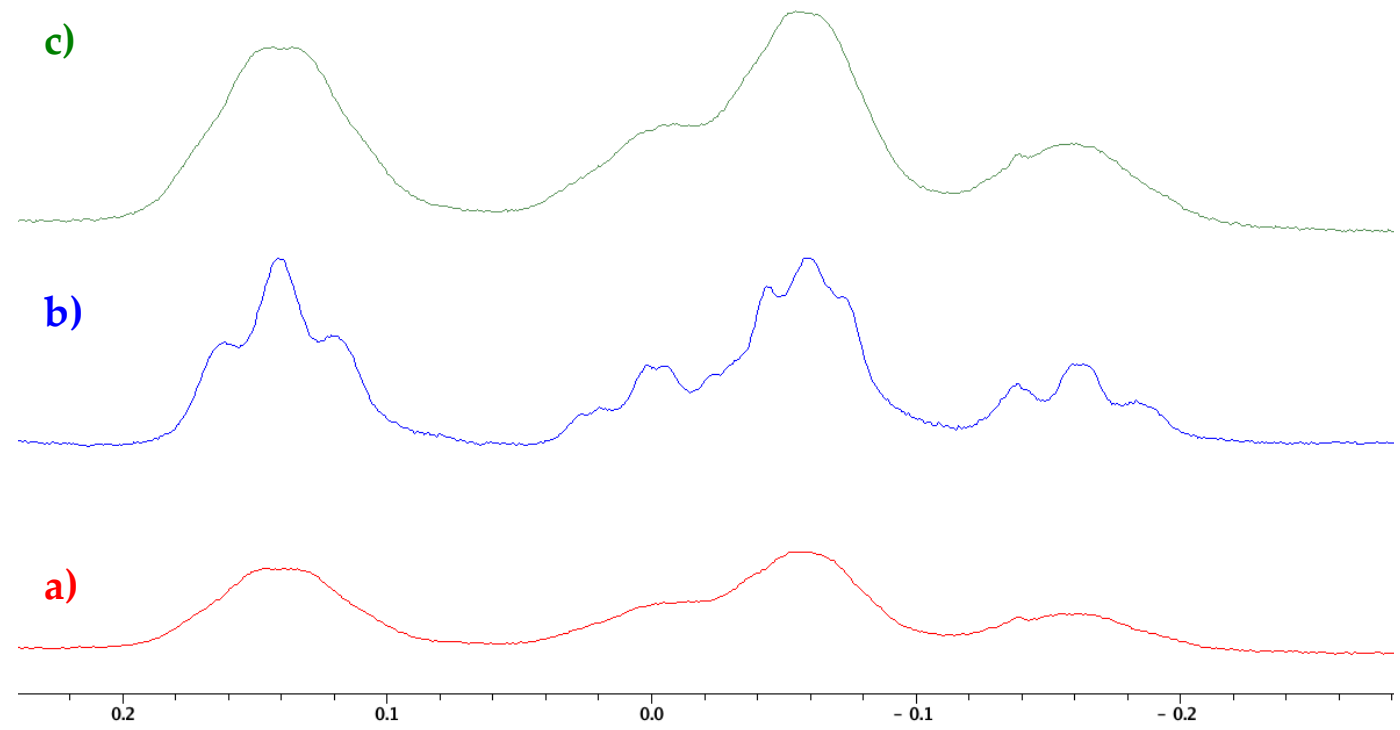
Figure S19. Treatment of $[3]^{+}$with 8 equivs. $n$-BuLi, ${ }^{1} \mathrm{H}\left\{{ }^{11} \mathrm{~B}\right\}$ NMR, THF- $\mathrm{d}_{8}, 500 \mathrm{MHz}, 298$ $\mathrm{K}$. This spectrum shows the four $\mathrm{CH} / \mathrm{CH}_{2}$ groups $\alpha$ to the borate group $(48 \mathrm{H})$.

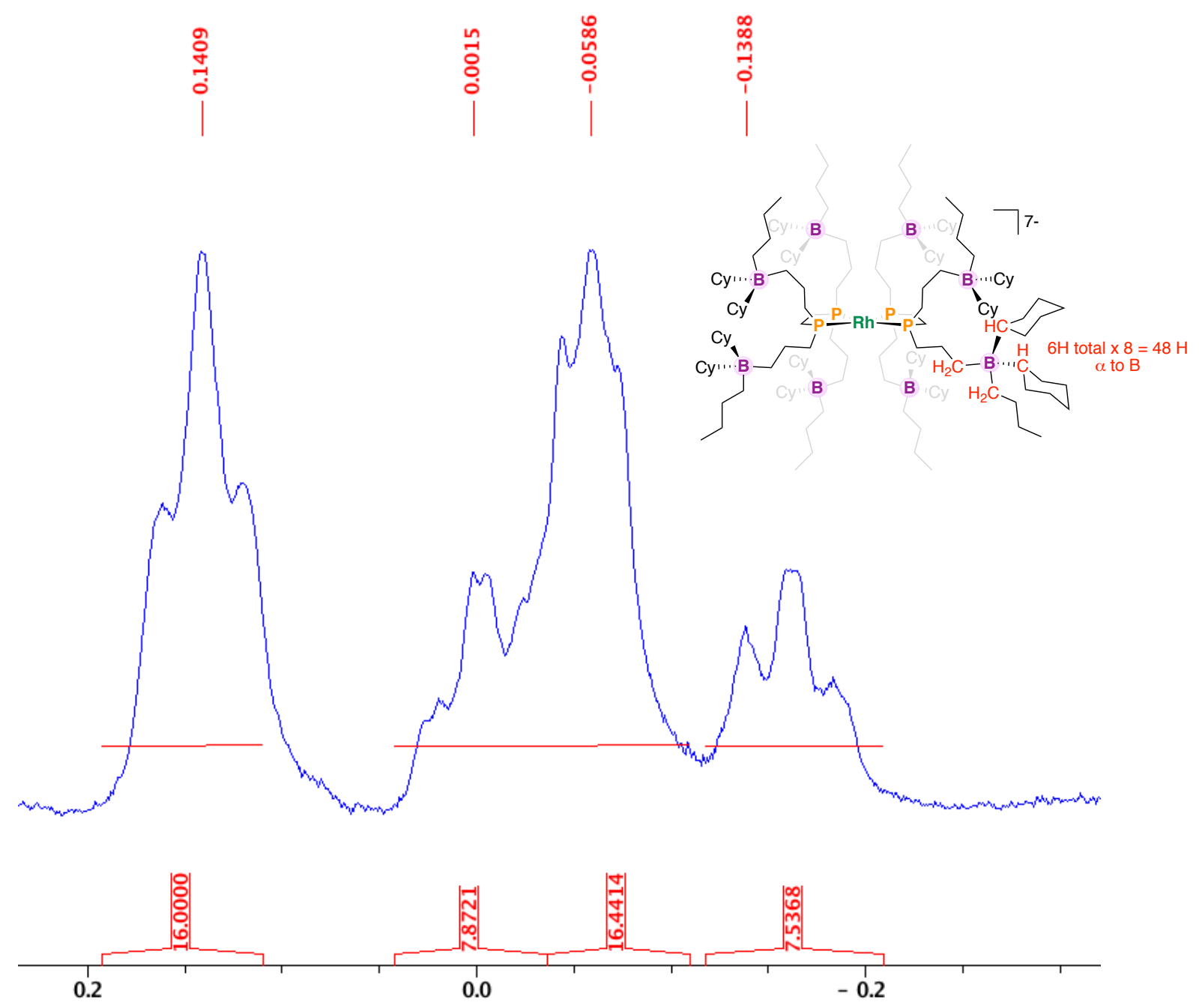


Figure S20. Treatment of [3] ${ }^{+}$with 8 equivs. ${ }^{n} \mathrm{BuLi},{ }^{31} \mathrm{P}\left\{{ }^{1} \mathrm{H}\right\} \mathrm{NMR}, \mathrm{THF}-\mathrm{d}_{8}, 203 \mathrm{MHz}, 298$ K.

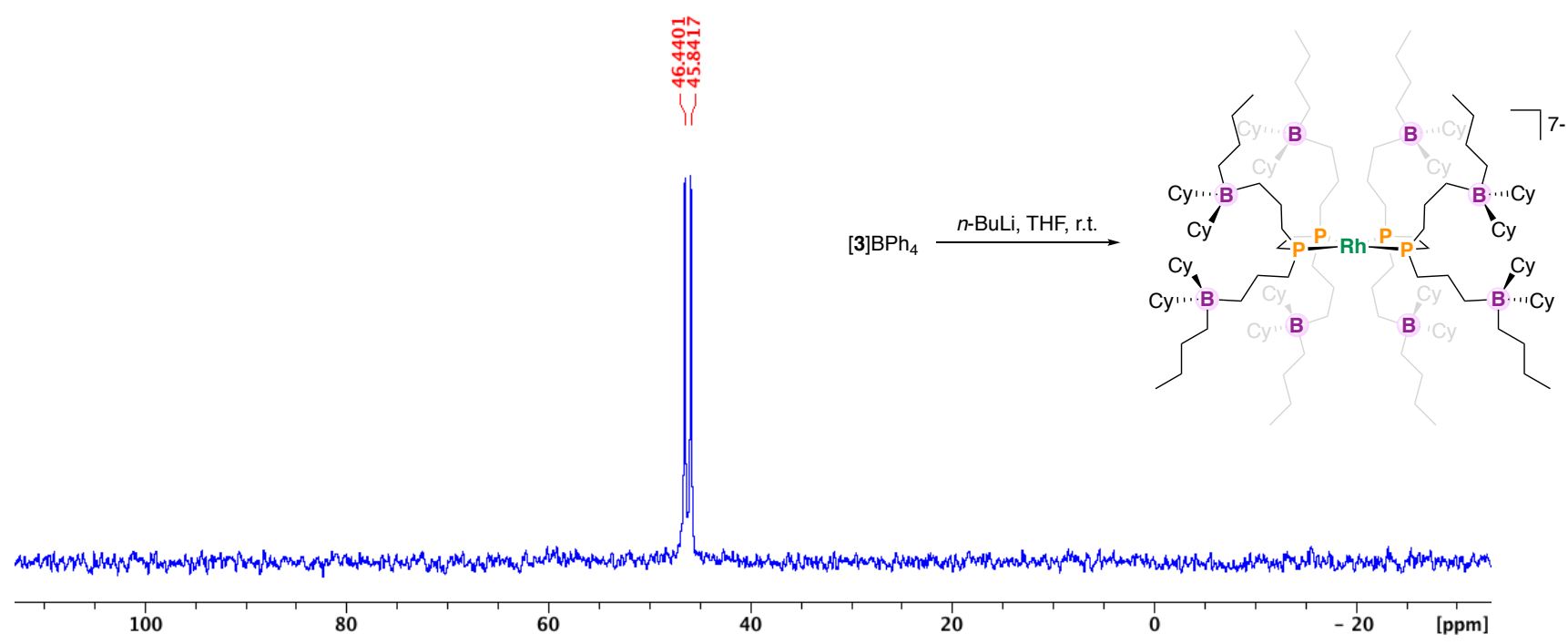

Figure S21. Treatment of $[3]^{+}$with 8 equivs. ${ }^{n} \mathrm{BuLi},{ }^{11} \mathrm{~B}\left\{{ }^{1} \mathrm{H}\right\} \mathrm{NMR}, \mathrm{THF}-\mathrm{d}_{8}, 161 \mathrm{MHz}, 298$ K.

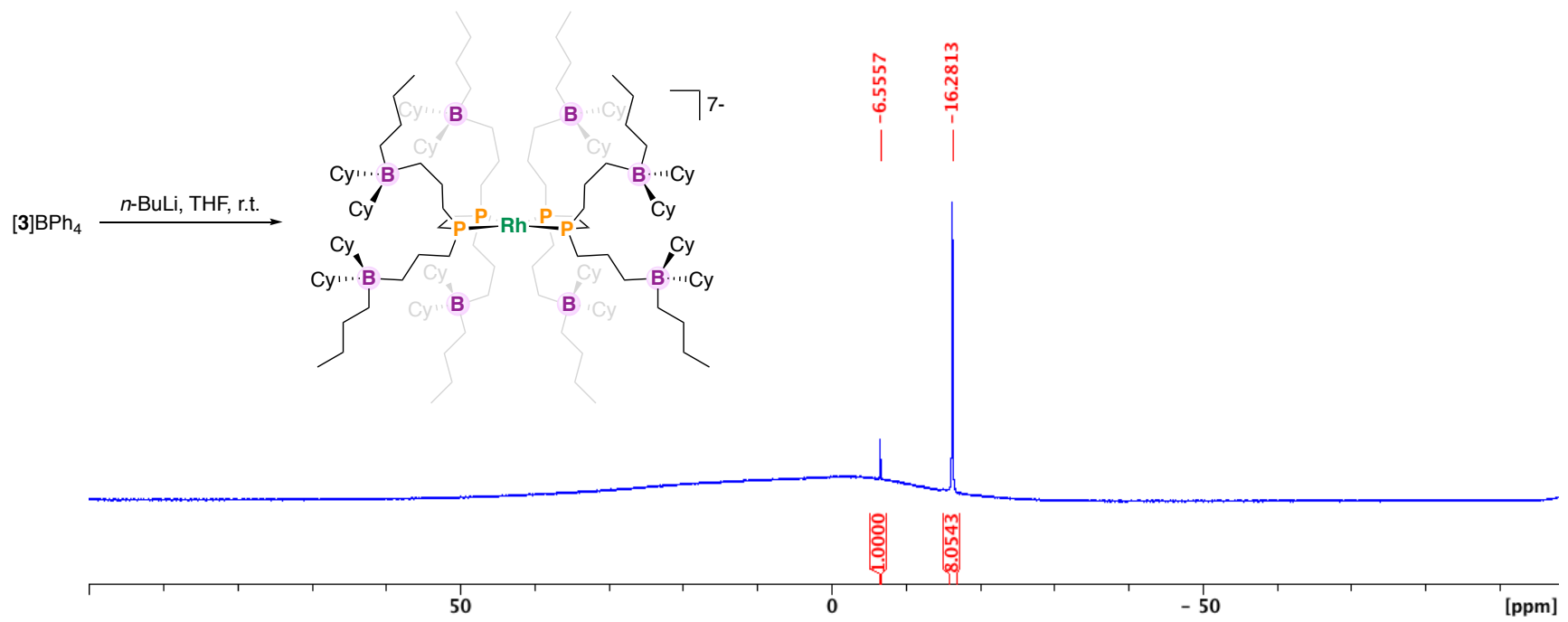


Figure S22. 5, ${ }^{1} \mathrm{H}$ NMR, THF-d $8,500 \mathrm{MHz}, 298 \mathrm{~K}$.

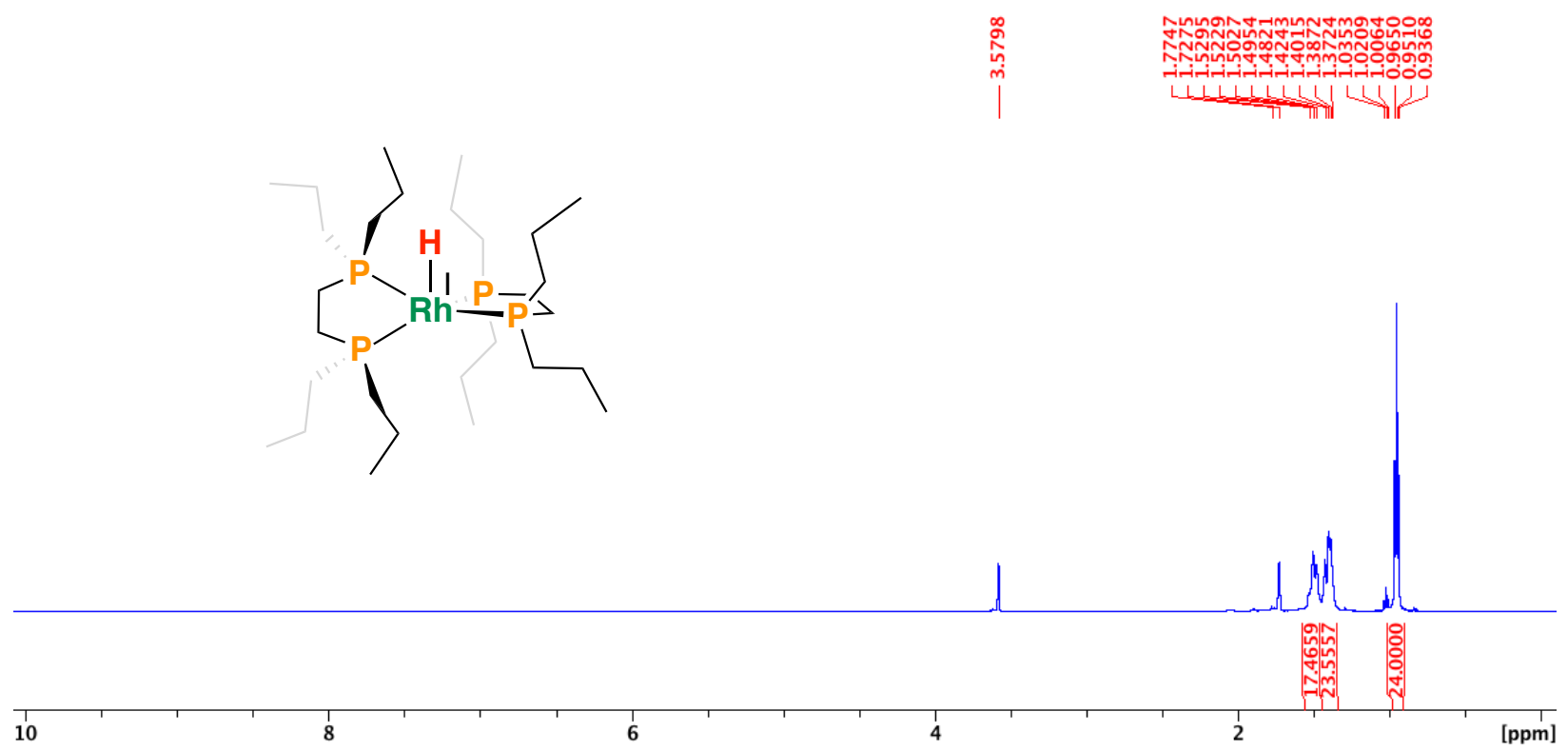

Figure S23. 5, a) ${ }^{1} \mathrm{H}$ and b) ${ }^{1} \mathrm{H}\left\{{ }^{31} \mathrm{P}\right\} \mathrm{NMR}, \mathrm{THF}-\mathrm{d}_{8}, 500 \mathrm{MHz}, 298 \mathrm{~K}$.

b)

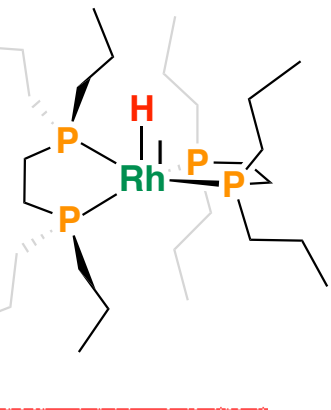

a) 
Figure S24. 5, ${ }^{31} \mathrm{P}\left\{{ }^{1} \mathrm{H}\right\} \mathrm{NMR}, \mathrm{THF}-\mathrm{d}_{8}, 203 \mathrm{MHz}, 298 \mathrm{~K}$.

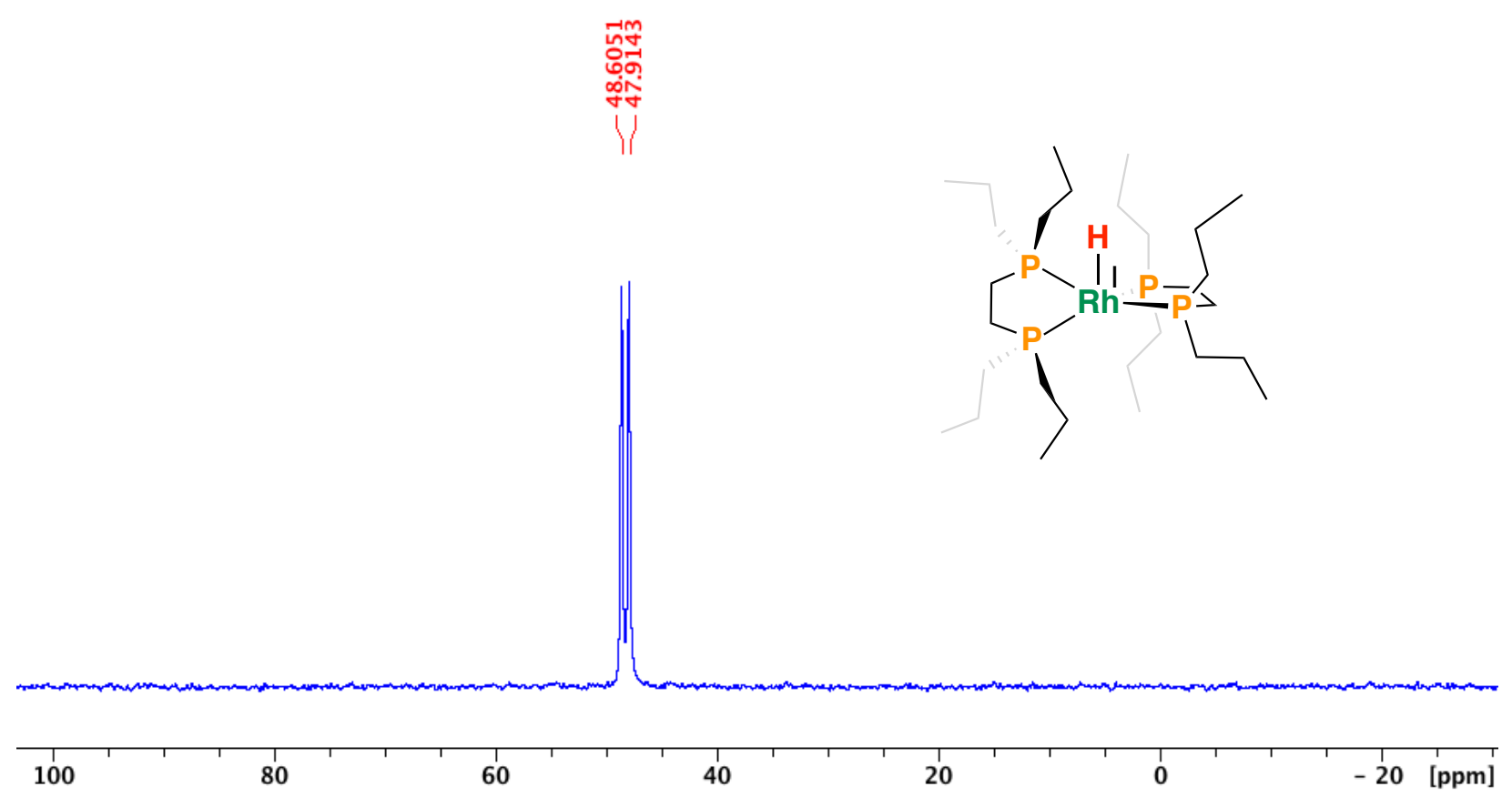

Figure S25. 5, ${ }^{13} \mathrm{C}\left\{{ }^{1} \mathrm{H}\right\} \mathrm{NMR}, \mathrm{THF}-\mathrm{d}_{8}, 125 \mathrm{MHz}, 298 \mathrm{~K}$.

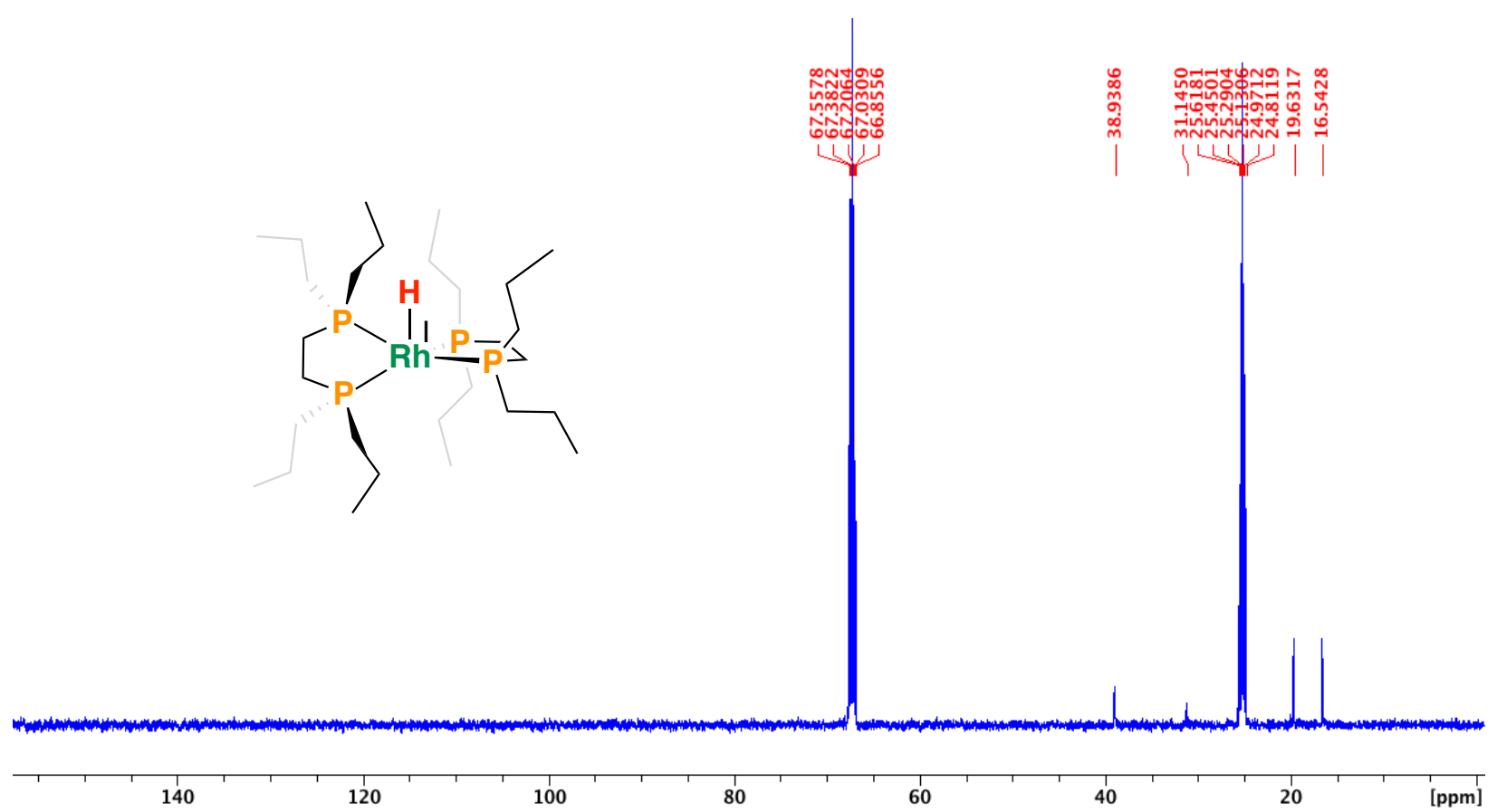


Figure S26. a) $[3]^{+}$and b) treatment of $[3]^{+}$with excess (> 50 equivs.) K[HBEt 3$],{ }^{1} \mathrm{H}$ NMR, THF- $\mathrm{d}_{8}, 500 \mathrm{MHz}, 298 \mathrm{~K}$. Structure of $[6]^{7-}$ shown to suggest potential interactions only.

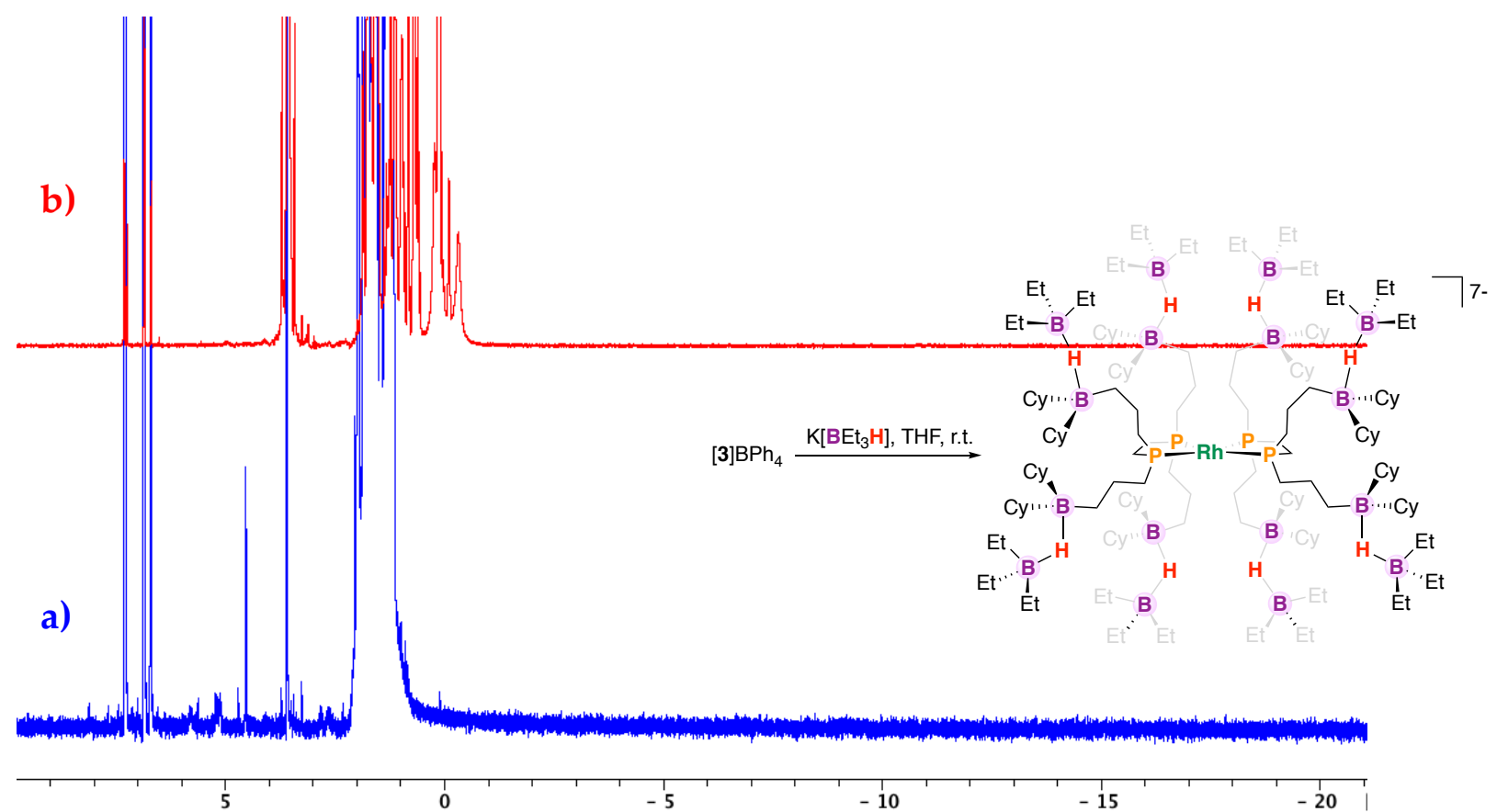

Figure S27. a) $[3]^{+}$and b) treatment of $[3]^{+}$with excess (> 50 equivs.) K[HBEt3], ${ }^{1} \mathrm{H} \mathrm{NMR}$, THF- $\mathrm{d}_{8}, 500 \mathrm{MHz}, 298 \mathrm{~K}$ (expansion). Structure of [6] $]^{7-}$ shown to suggest potential interactions only.

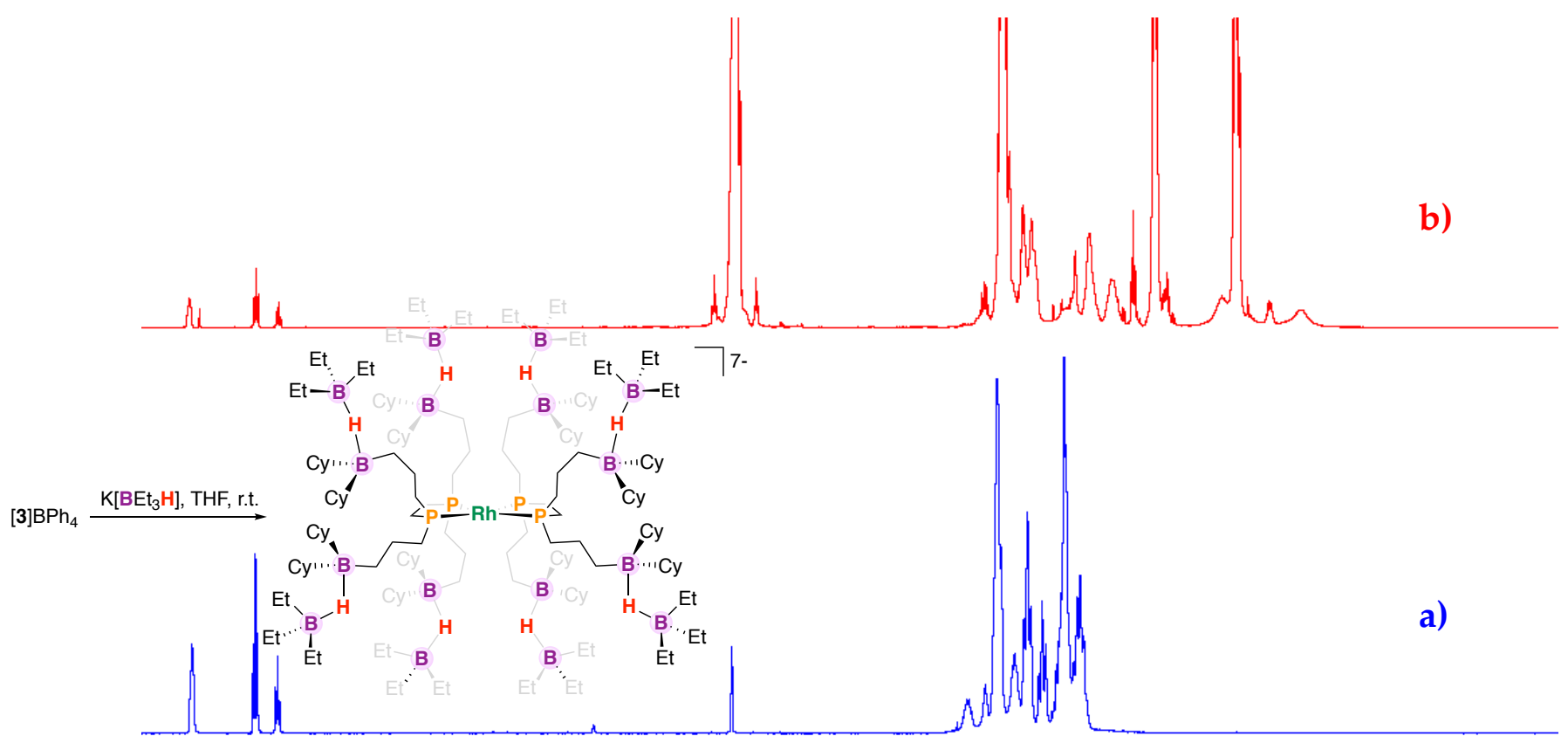


Figure S28. Treatment of [3] $]^{+}$with excess (> 50 equivs.) K[HBEt 3$],{ }^{31} \mathrm{P}\left\{{ }^{1} \mathrm{H}\right\} \mathrm{NMR}, \mathrm{THF}-\mathrm{d}_{8}$, $203 \mathrm{MHz}, 298 \mathrm{~K}$. Structure of [6] $]^{7-}$ shown to suggest potential interactions only.

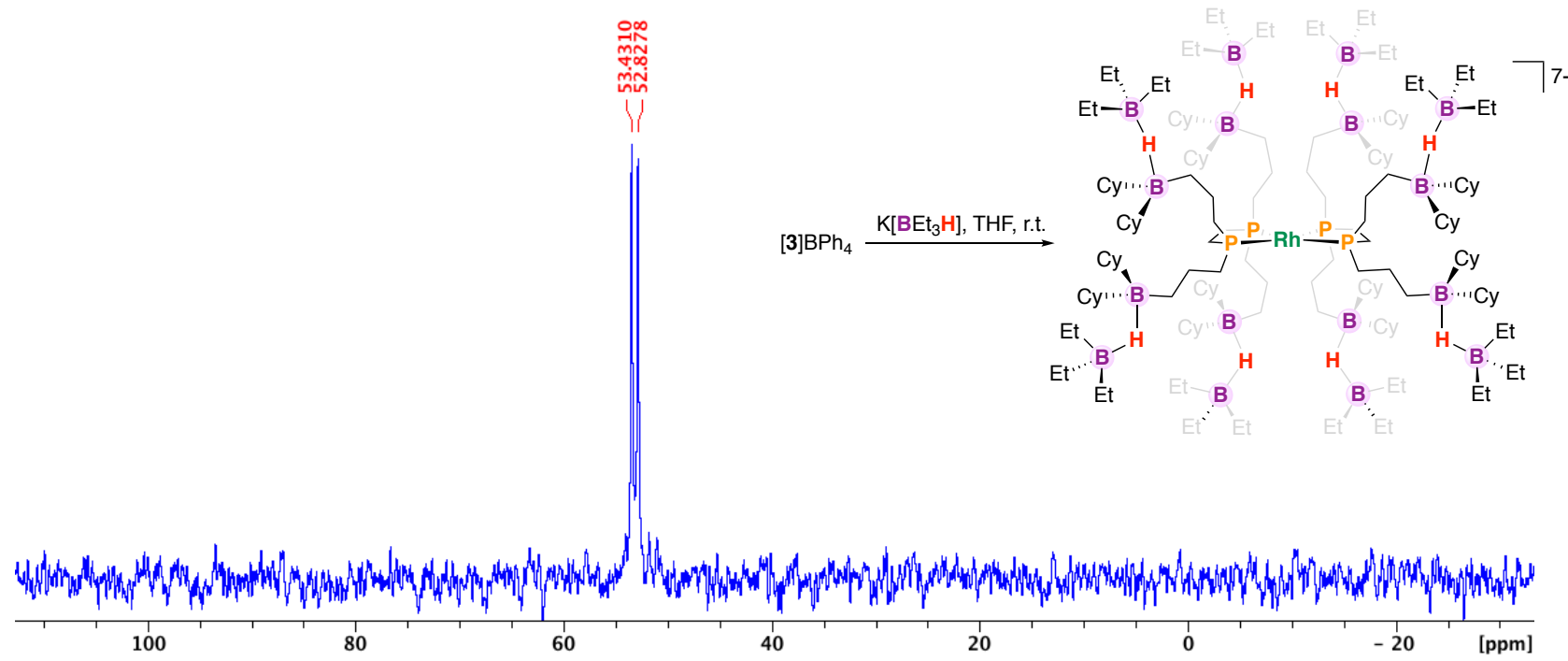

Figure S29. Treatment of $[3]^{+}$with excess (> 50 equivs.) K[HBEt 3$],{ }^{11} \mathrm{~B}\left\{{ }^{1} \mathrm{H}\right\} \mathrm{NMR}, \mathrm{THF}-\mathrm{d}_{8}$, $161 \mathrm{MHz}, 298 \mathrm{~K}$. Reaction of $\mathrm{K}\left[\mathrm{HBEt}_{3}\right]$ with $\mathrm{BEt}_{3}$ gives a signal at $\delta_{\mathrm{B}}=7.5 \mathrm{ppm}^{1}$ Structure of $[6]^{7-}$ shown to suggest potential interactions only.

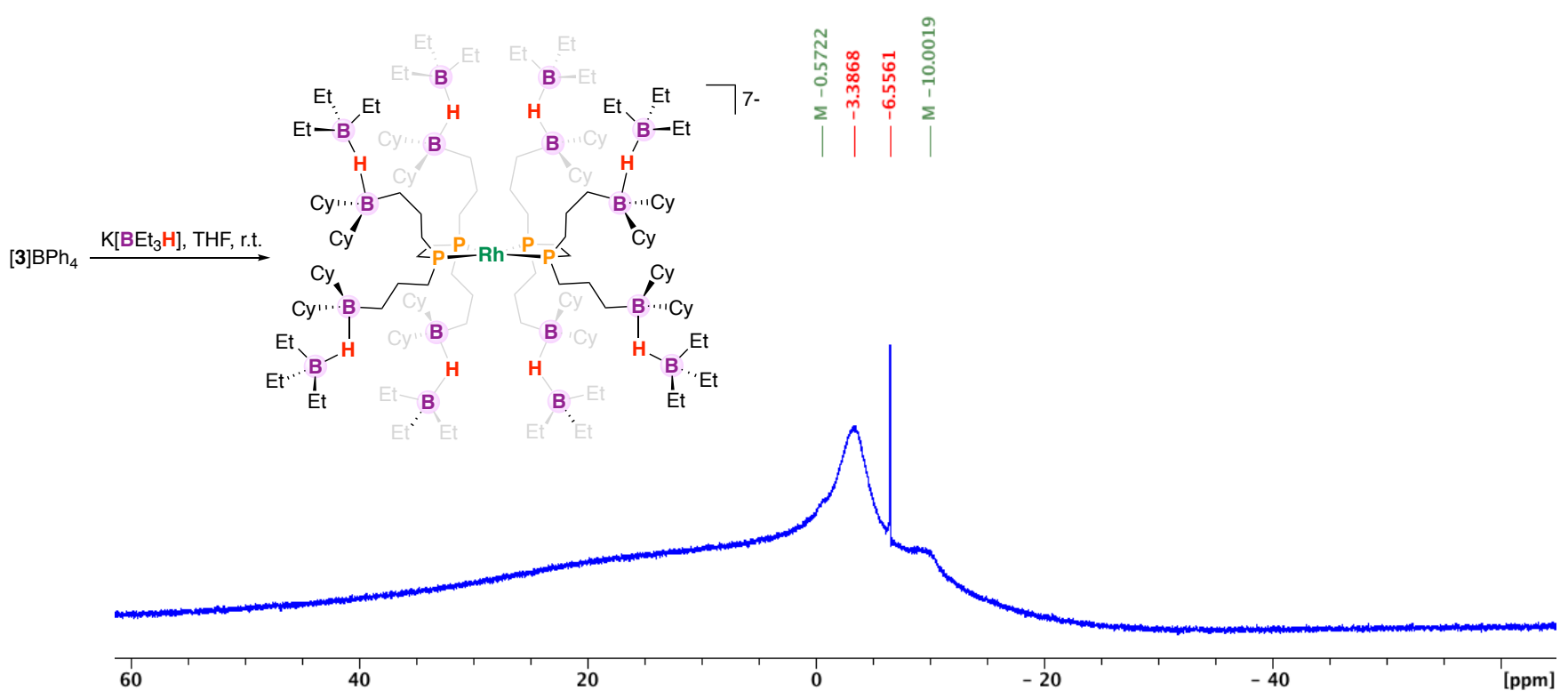

${ }^{1}$ C. A. Brown, J. Organomet. Chem. 1978, 156, C17. 
Figure S30. Treatment of $[3]^{+}$with $1-50$ equivs. K[HBEt 3$],{ }^{31} \mathrm{P}\left\{{ }^{1} \mathrm{H}\right\} \mathrm{NMR}, \mathrm{THF}-\mathrm{d}_{8}, 203$ $\mathrm{MHz}, 298 \mathrm{~K}$.

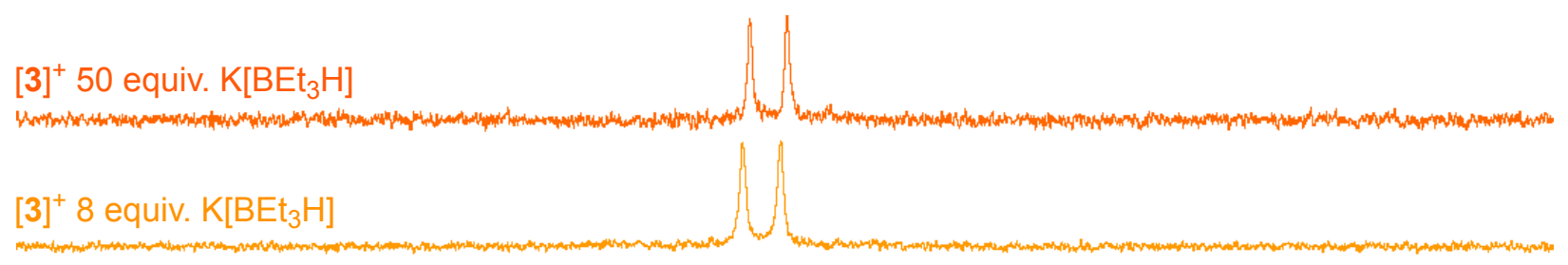

$[3]^{+} 4$ equiv. $\mathrm{K}\left[\mathrm{BEE} \mathrm{T}_{3} \mathrm{H}\right]$

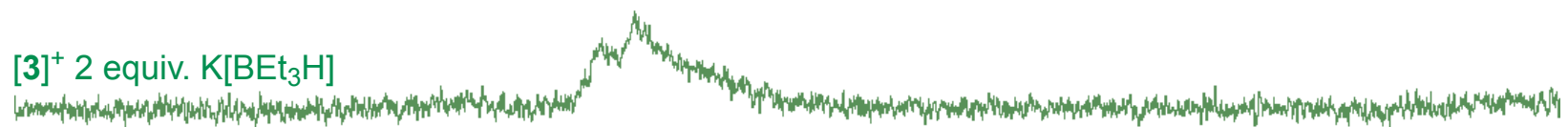
[3] 1 equiv. K[BEt $\left.]_{3} \mathrm{H}\right]$ $[3]^{+}$

Figure S31. Treatment of $[3]^{+}$with 1 - 50 equivs. $\mathrm{K}\left[\mathrm{HBEt}_{3}\right],{ }^{11} \mathrm{~B}\left\{{ }^{1} \mathrm{H}\right\} \mathrm{NMR}$, THF- $\mathrm{d}_{8}, 161$ $\mathrm{MHz}, 298 \mathrm{~K}$.

$[3]^{+} 50$ equiv. $\mathrm{K}\left[\mathrm{BEt}_{3} \mathrm{H}\right]$
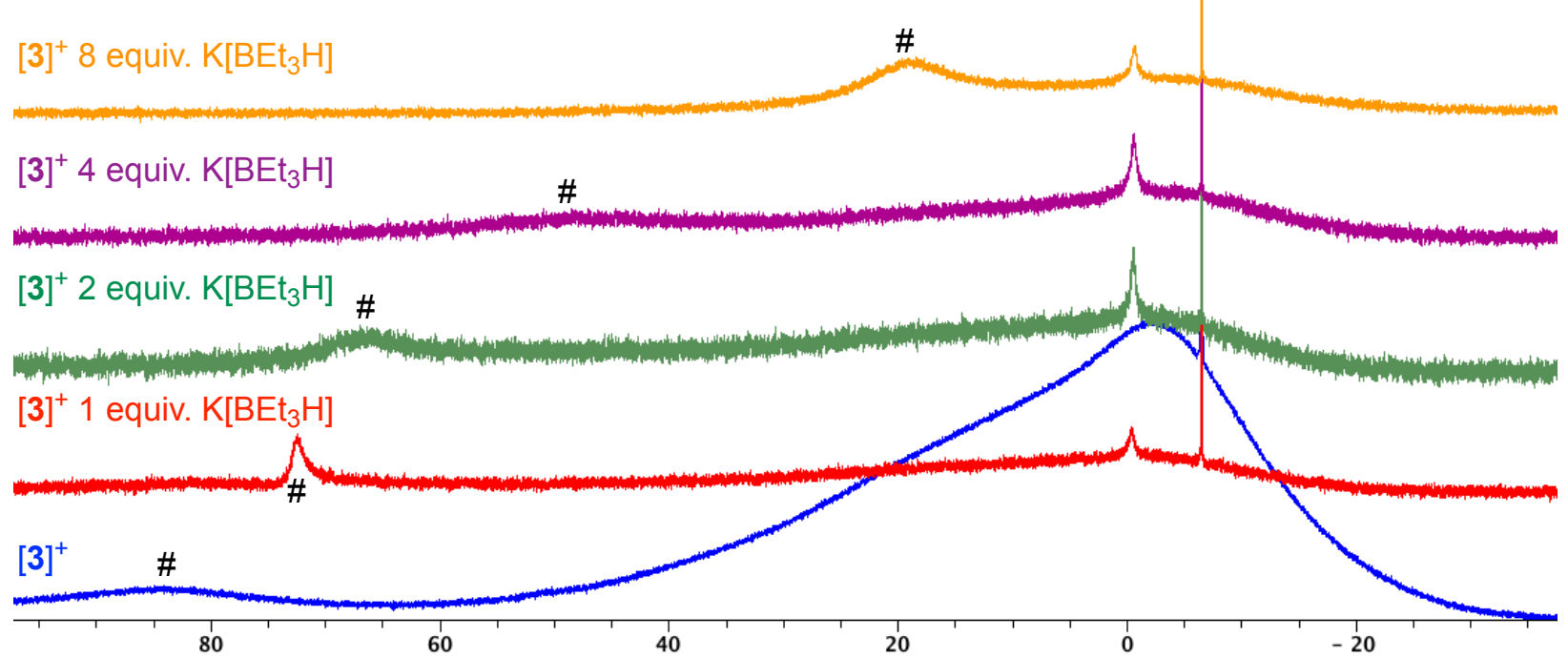
Figure S32. Treatment of $[3]^{+}$with ${ }^{n} \mathrm{BuLi}$ (left) or K[HBEt 3$]$ (right) in THF-d8. For treatment with $\mathrm{K}\left[\mathrm{HBEt}_{3}\right]$, a white cloudy precipitate is formed and settles at the bottom of the tube. Structure of $[6]^{7-}$ shown to suggest potential interactions only.

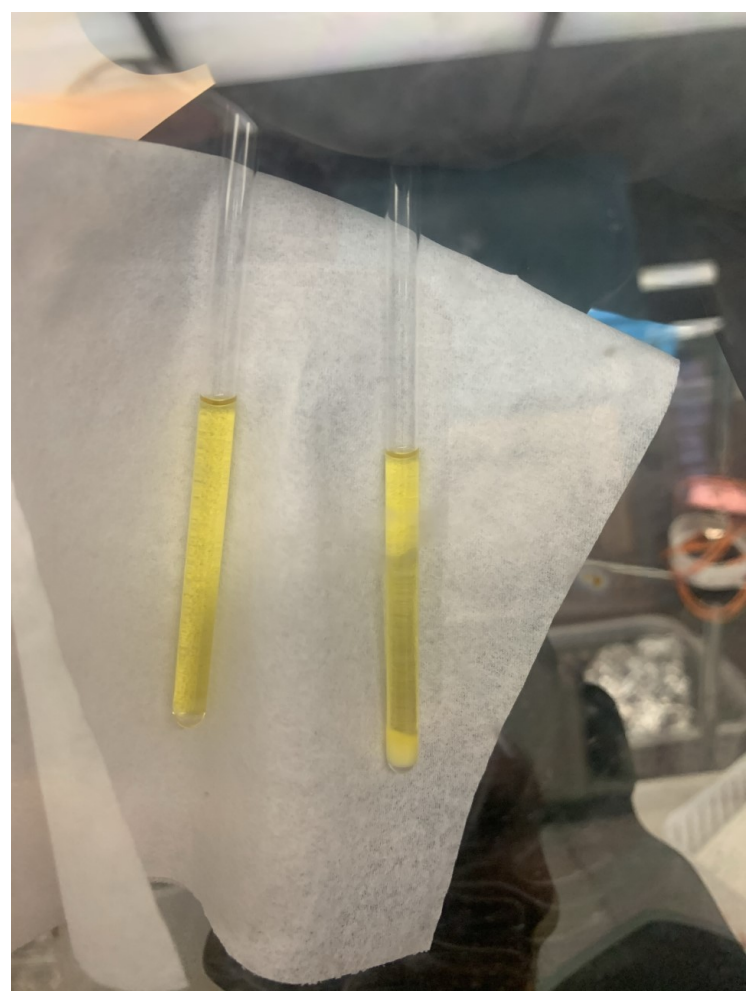

$[3] \mathrm{BPh}_{4} \underset{\text { LEFT NMR TUBE }}{\stackrel{n-\mathrm{BuLi}, \text { THF, r.t. }}{\longrightarrow}}$

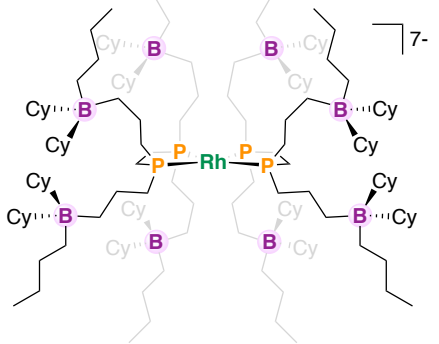

[3] $\mathrm{BPh}_{4} \underset{\text { RIGHT NMR TUBE }}{\stackrel{\mathrm{K}\left[\mathrm{BEt}_{3} \mathrm{H}\right], \text { THF, r.t. }}{\longrightarrow}}$

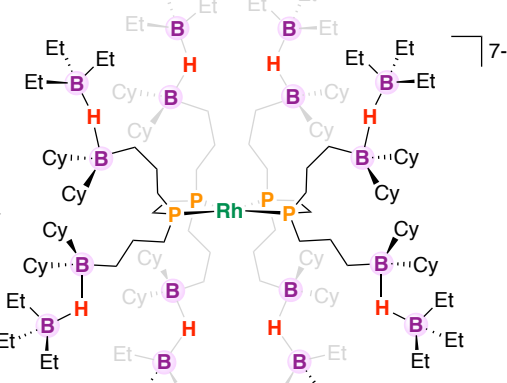


Figure S33. Treatment of $[1]^{+}$with ${ }^{n} \mathrm{BuLi},{ }^{31} \mathrm{P}\left\{{ }^{1} \mathrm{H}\right\} \mathrm{NMR}, \mathrm{THF}-\mathrm{d}_{8}, 203 \mathrm{MHz}, 298 \mathrm{~K}$.

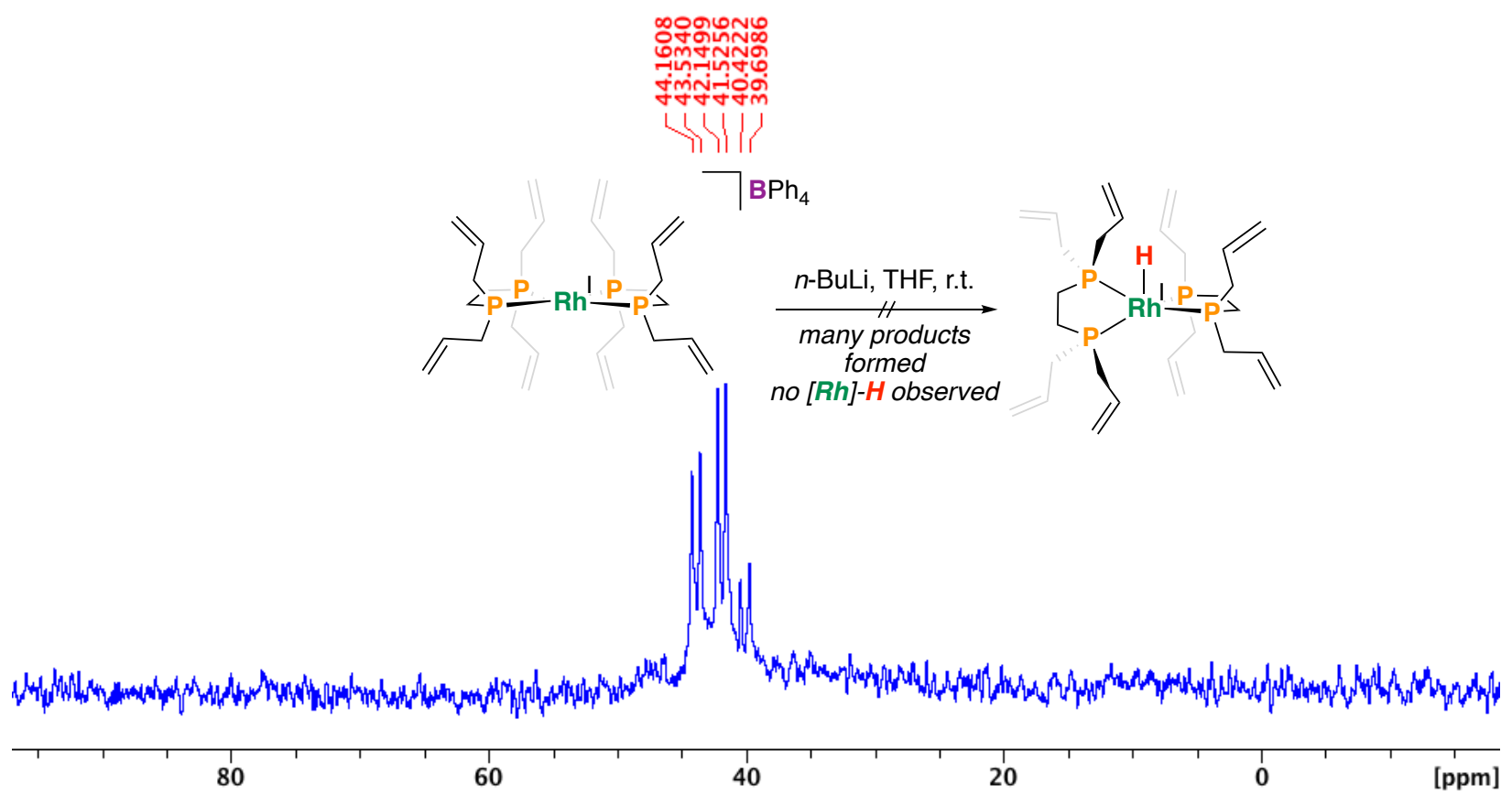

Figure S34. Treatment of [2] ${ }^{+}$with $\mathrm{K}\left[\mathrm{HBEt}{ }_{3}\right],{ }^{31} \mathrm{P}\left\{{ }^{1} \mathrm{H}\right\} \mathrm{NMR}, \mathrm{THF}-\mathrm{d}_{8}, 203 \mathrm{MHz}, 298 \mathrm{~K}$.

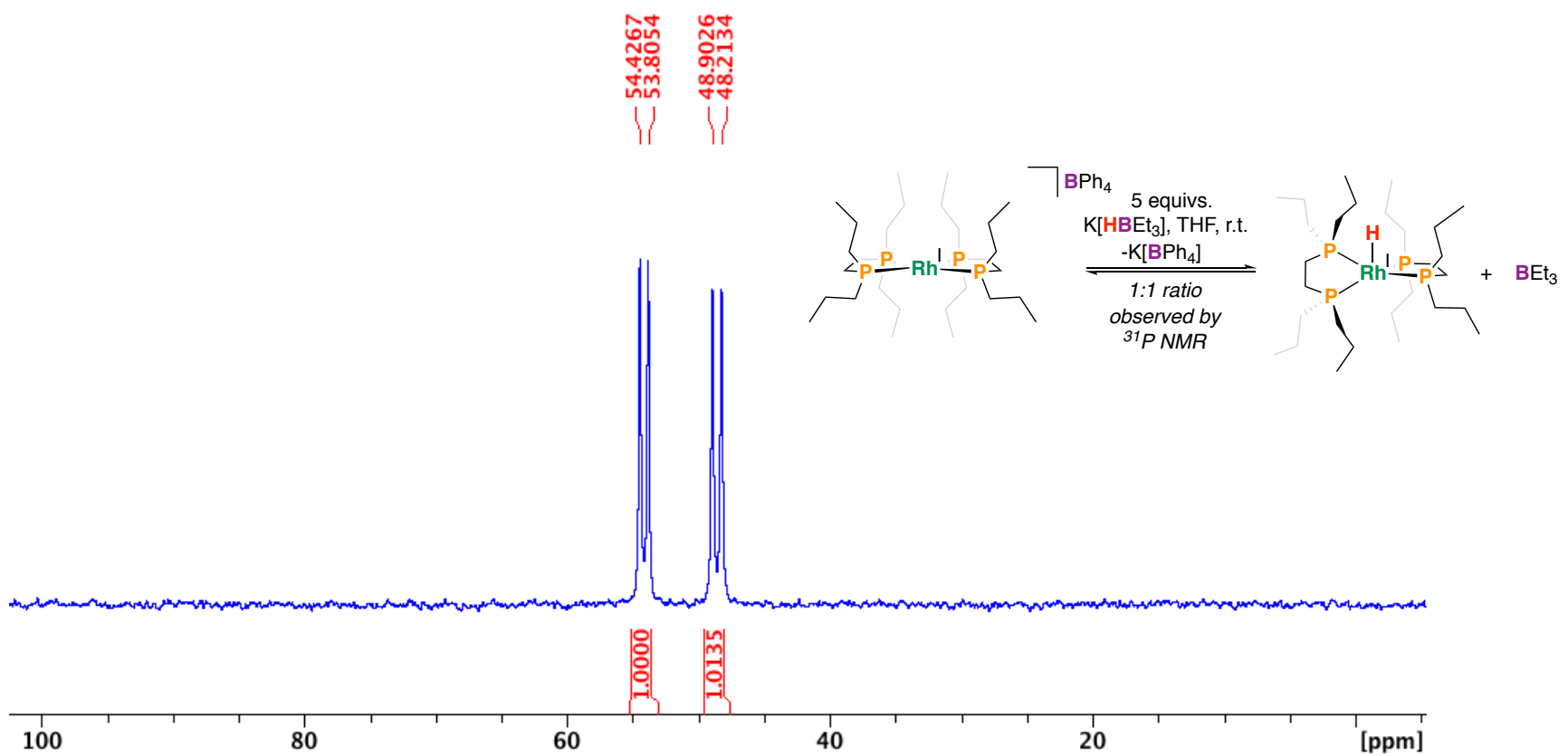


Figure S35. Treatment of [2] ${ }^{+}$with $\mathrm{K}\left[\mathrm{HBEt}_{3}\right],{ }^{11} \mathrm{~B}\left\{{ }^{1} \mathrm{H}\right\} \mathrm{NMR}, \mathrm{THF}-\mathrm{d}_{8}, 161 \mathrm{MHz}, 298 \mathrm{~K}$.

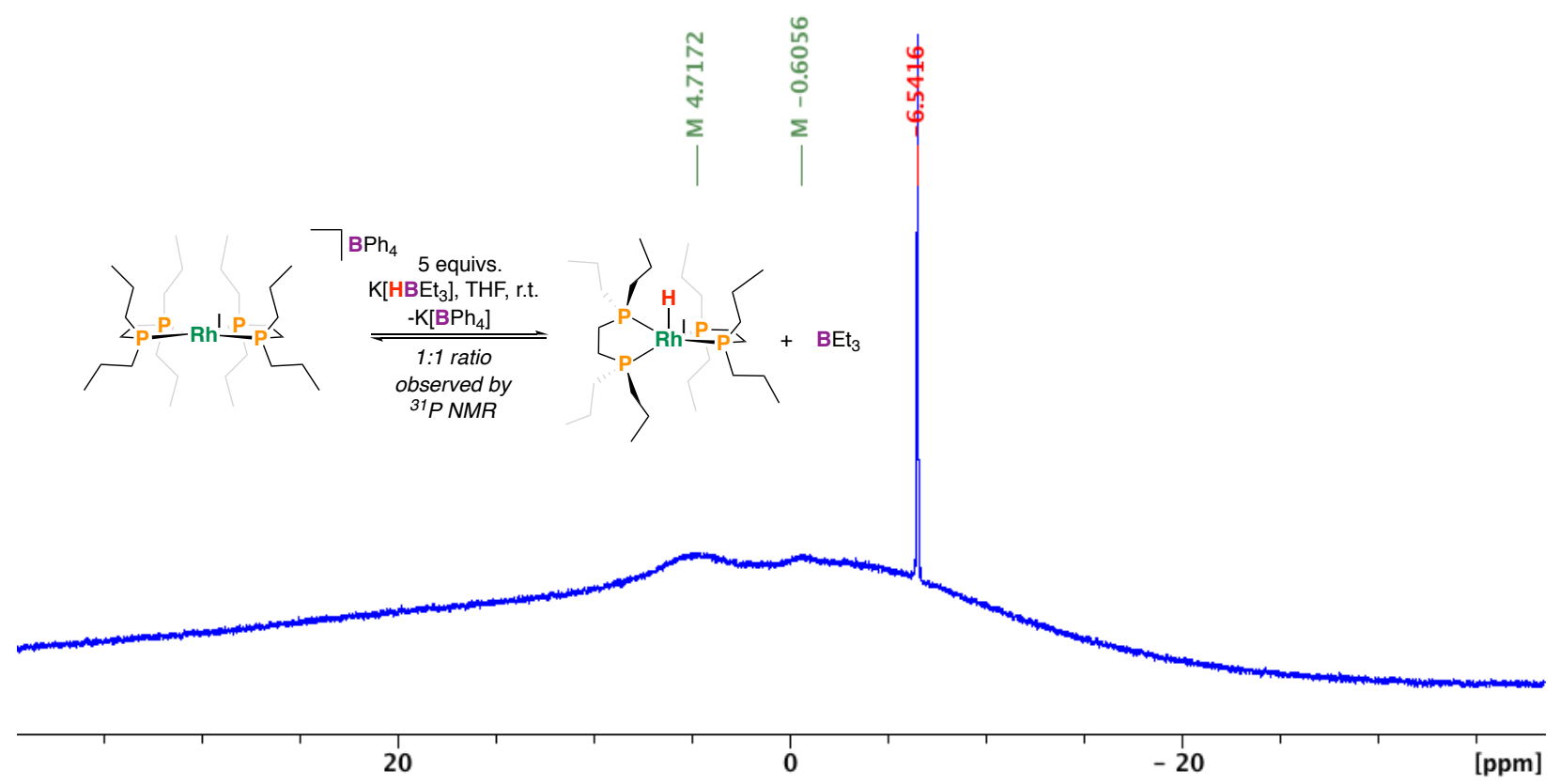

Figure S36. K[HBEt 3$\left.],{ }^{1} \mathrm{H}_{\{}{ }^{11} \mathrm{~B}\right\}$ NMR proteo THF, $500 \mathrm{MHz}, 298 \mathrm{~K}$.

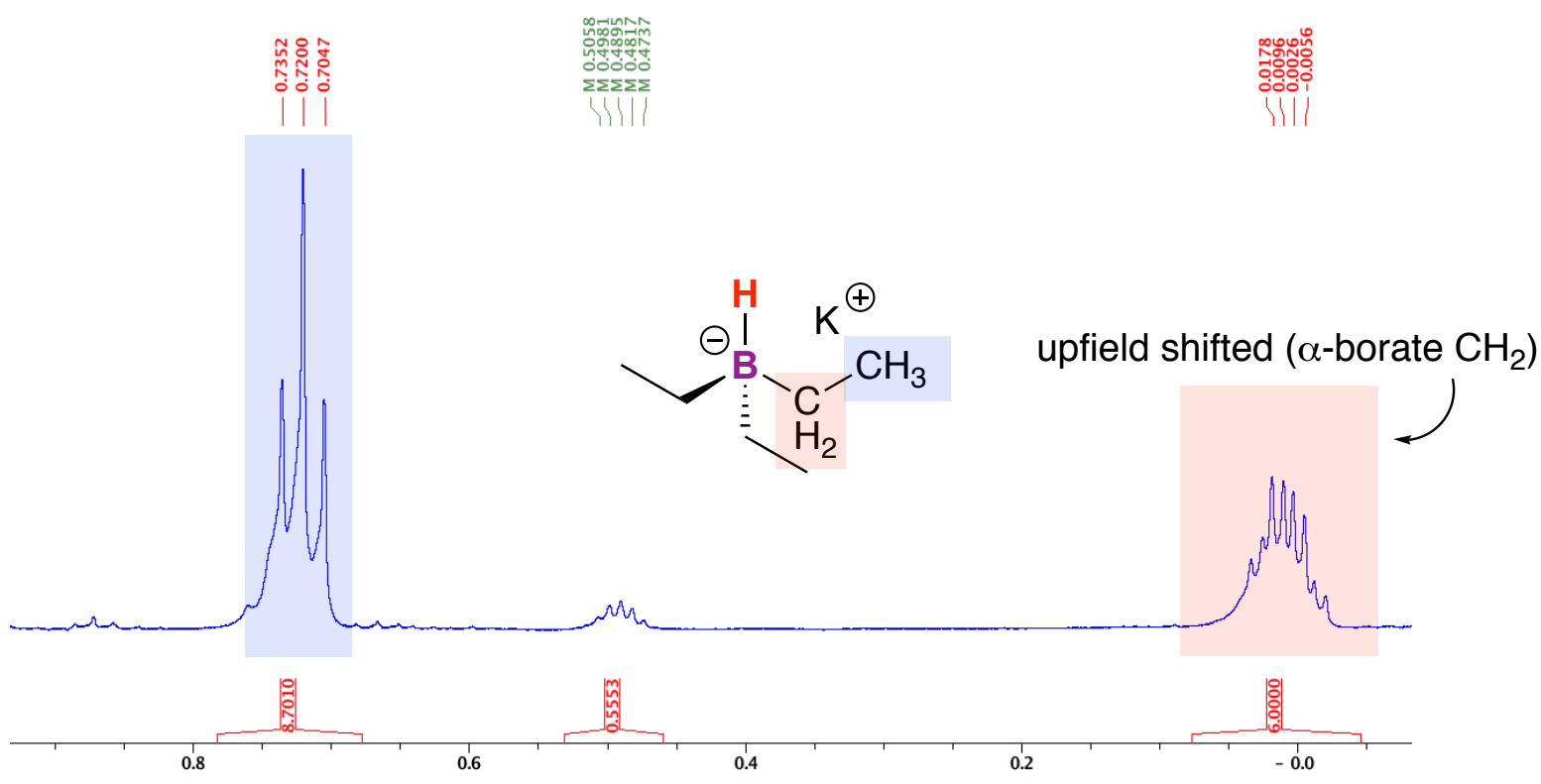


Figure S37. $\mathrm{K}\left[\mathrm{HBEt}_{3}\right]$, a) $\left.{ }^{1} \mathrm{H} \mathrm{NMR}, \mathrm{b}\right){ }^{1} \mathrm{H}\left\{{ }^{11} \mathrm{~B}\right\} \mathrm{NMR}$ (o2p = -12 ppm), proteo THF, 500 $\mathrm{MHz}, 298 \mathrm{~K}$. Et 3 B- $\underline{\mathrm{H}}$ resonance at $\delta_{\mathrm{H}} \sim 0.48 \mathrm{ppm}$.

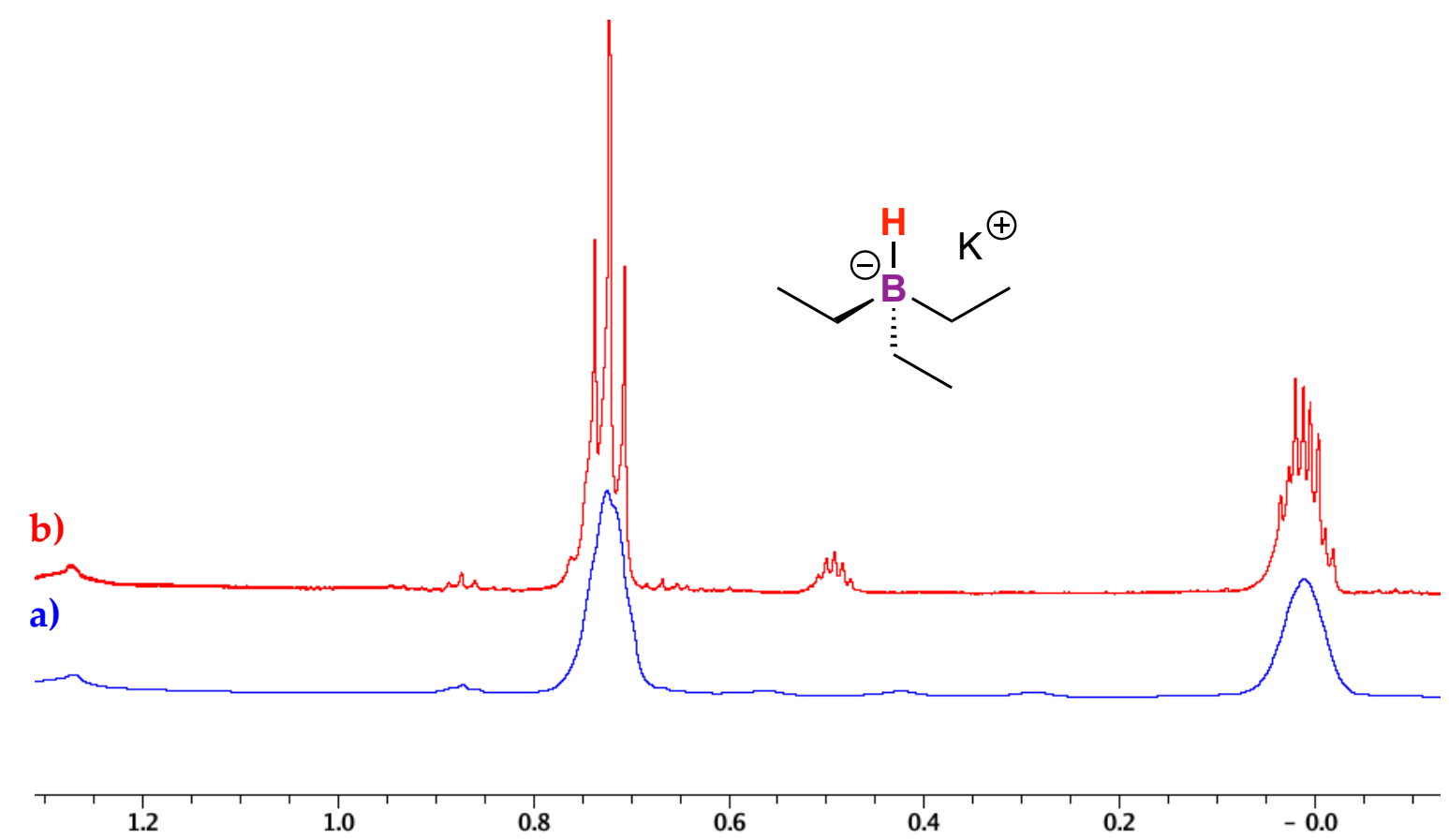

Figure S38. K[HBEt 3$],{ }^{11} \mathrm{~B}\left\{{ }^{1} \mathrm{H}\right\} \mathrm{NMR}$, proteo $\mathrm{THF}, 161 \mathrm{MHz}, 298 \mathrm{~K}$.

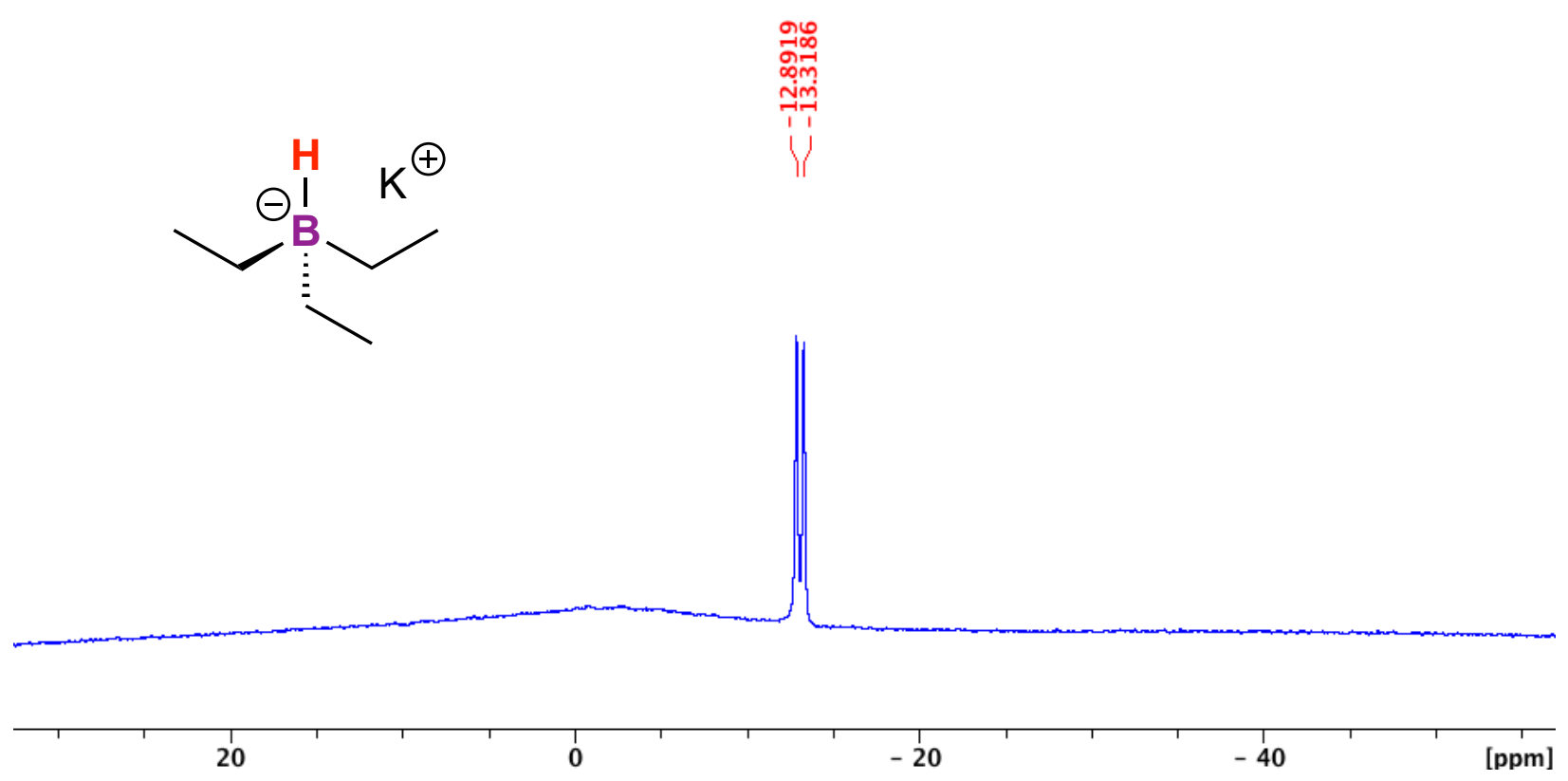


Figure S39. a) Treatment of $[3]^{+}$with $\left[\mathrm{Rh}(\operatorname{dnppe})_{2}(\mathrm{H})\right](5)$ and $\left.b\right)\left[\operatorname{Rh}(\operatorname{dnppe})_{2}\right]^{+}\left([2]^{+}\right)$ showing no hydride formation, ${ }^{1} \mathrm{H}$ NMR, THF- $\mathrm{d}_{8}, 500 \mathrm{MHz}, 298 \mathrm{~K}$.

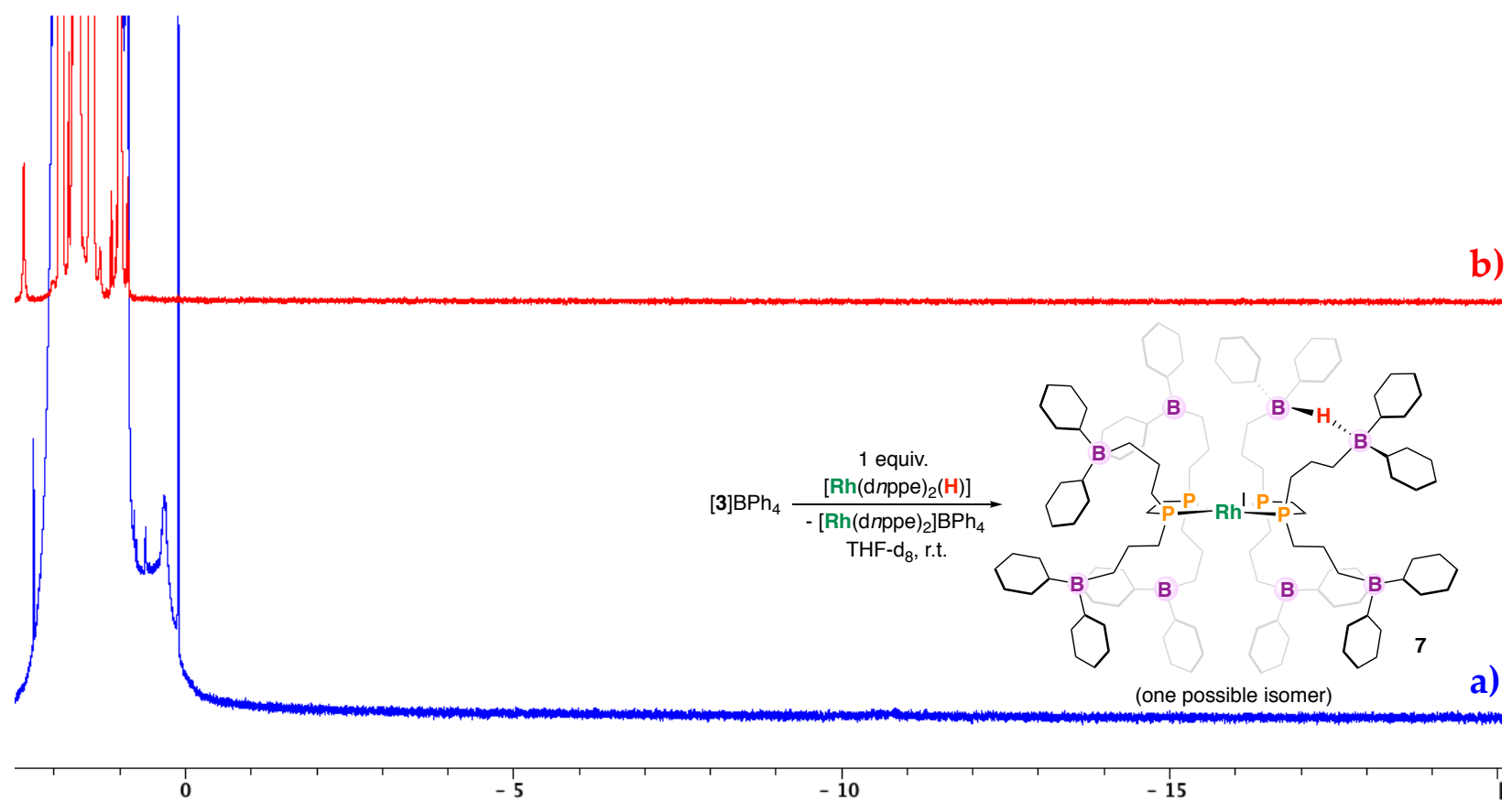

1

Figure S40. a) Treatment of $[3]^{+}$with $\left[\mathrm{Rh}(\text { dnppe })_{2}(\mathrm{H})\right](5)$ and b) $\left[\mathrm{Rh}(\text { dnppe })_{2}\right]^{+}\left([2]^{+}\right),{ }^{1} \mathrm{H}$ NMR, THF-d $\mathrm{d}_{8}, 500 \mathrm{MHz}, 298 \mathrm{~K}$.

b)

a)
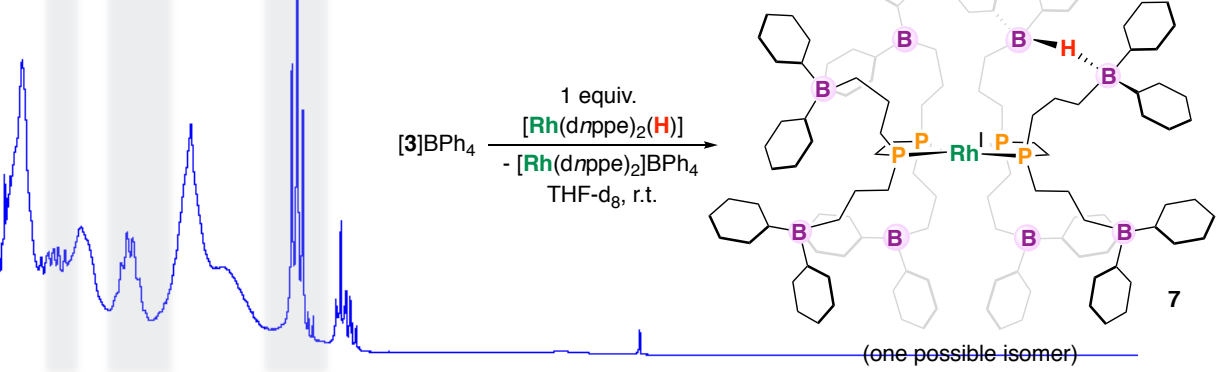

3 
Figure S41. a) $\left[\mathrm{Rh}(\operatorname{dnppe})_{2}(\mathrm{H})\right](5)$, b) treatment of $[3]^{+}$with $\left[\mathrm{Rh}(\operatorname{dnppe})_{2}(\mathrm{H})\right](5)$, and c) $\left.[\text { Rh(dnppe })_{2}\right]^{+}\left([2]^{+}\right),{ }^{31} \mathrm{P}\left\{{ }^{1} \mathrm{H}\right\} \mathrm{NMR}, \mathrm{THF}-\mathrm{d}_{8}, 203 \mathrm{MHz}, 298 \mathrm{~K}$.

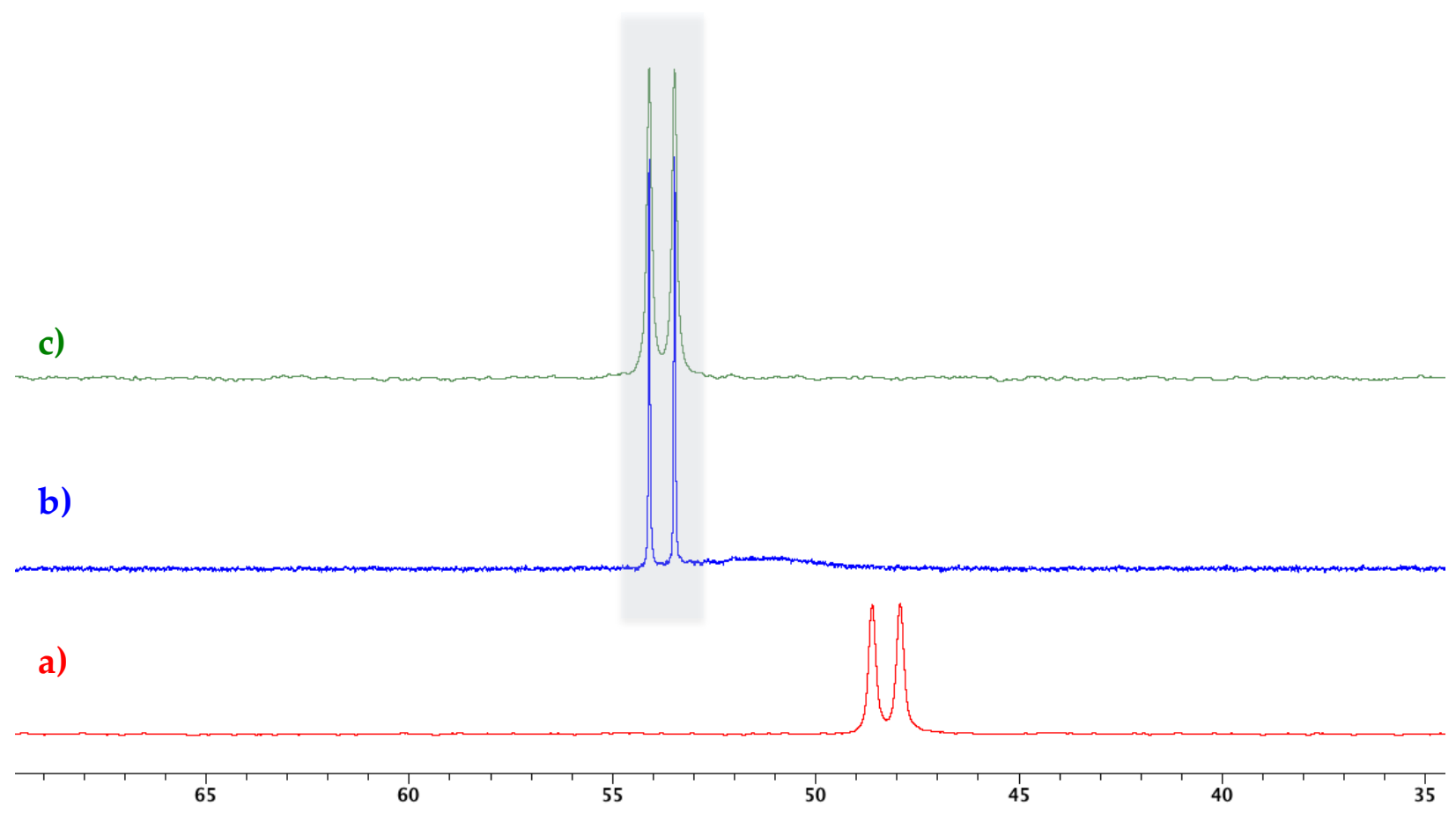

Figure S42. a) $[3]^{+}$and b) treatment of $[3]^{+}$with $\left[\operatorname{Rh}(\operatorname{dnppe})_{2}(\mathrm{H})\right](5),{ }^{11} \mathrm{~B}\left\{{ }^{1} \mathrm{H}\right\} \mathrm{NMR}$, THF$\mathrm{d}_{8}, 161 \mathrm{MHz}, 298 \mathrm{~K}$.

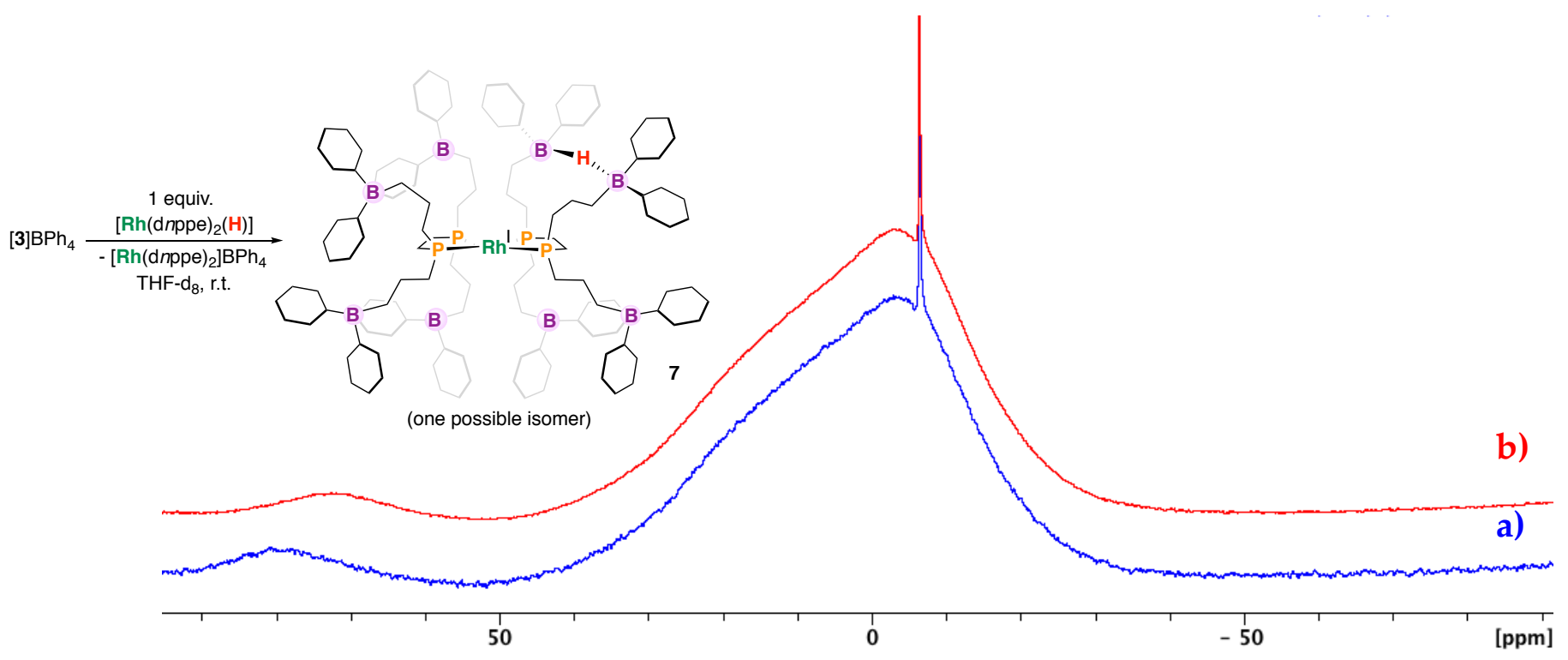


Figure S43. 7, ${ }^{1} \mathrm{H}$ NMR, THF-d $8,500 \mathrm{MHz}, 298 \mathrm{~K}$.

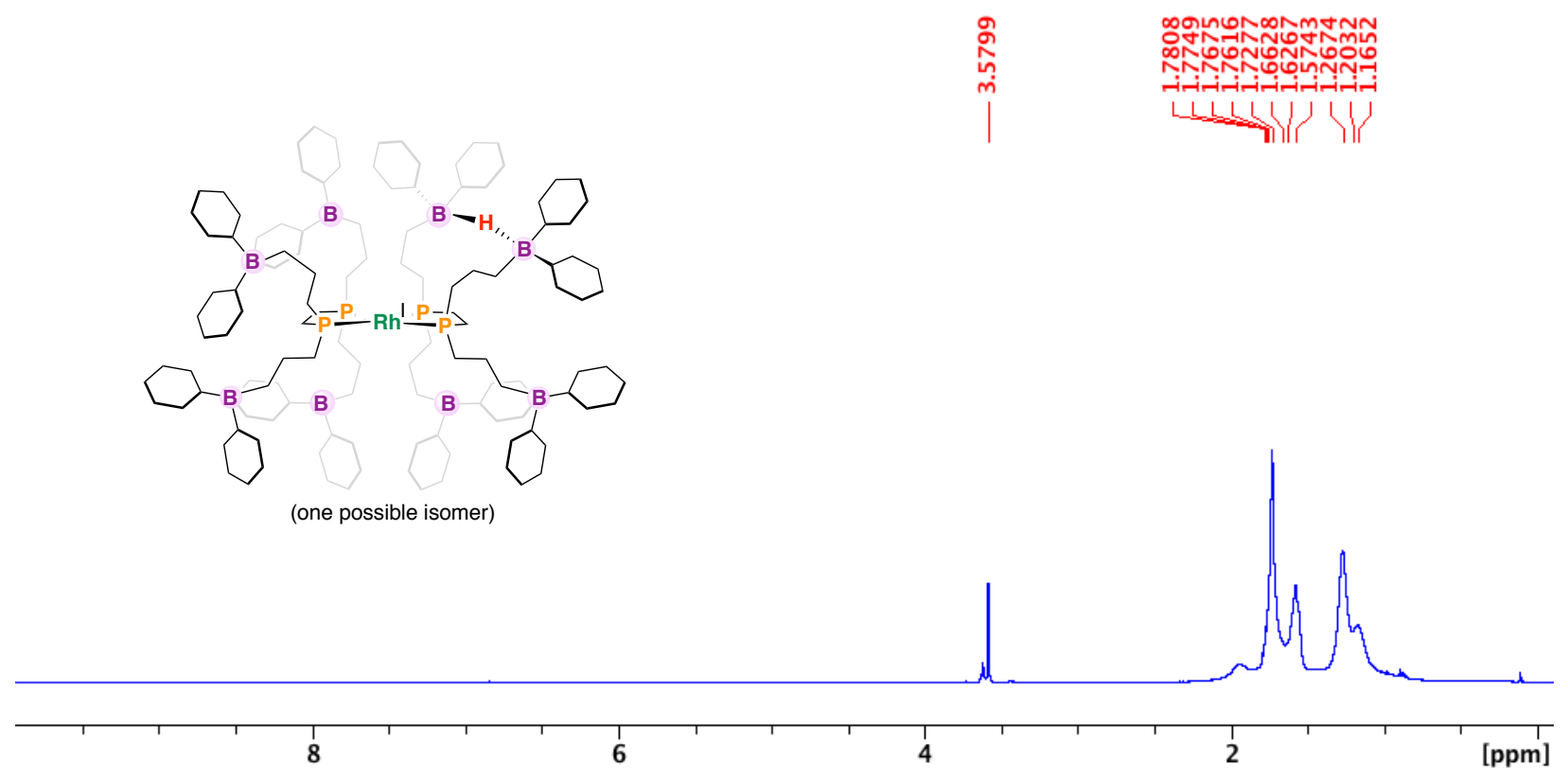

Figure S44. 7, ${ }^{31} \mathrm{P}\left\{{ }^{1} \mathrm{H}\right\} \mathrm{NMR}, \mathrm{THF}-\mathrm{d}_{8}, 203 \mathrm{MHz}, 298 \mathrm{~K}$.

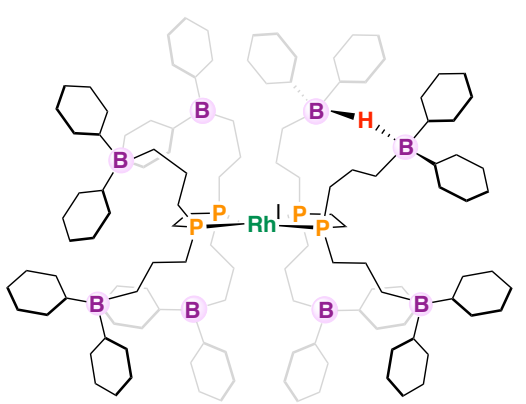

量

(one possible isomer)

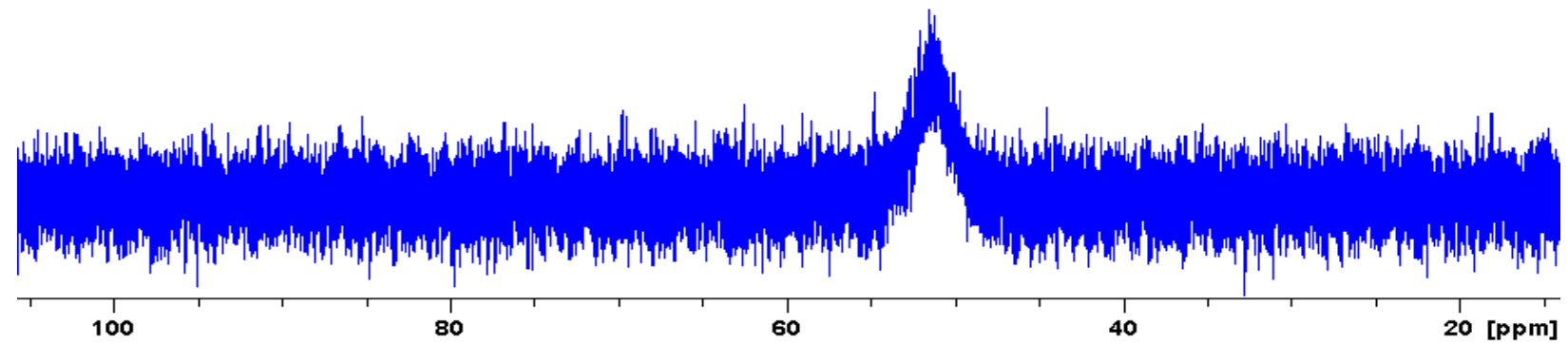


Figure S45. 7, ${ }^{11} \mathrm{~B}\left\{{ }^{1} \mathrm{H}\right\} \mathrm{NMR}, \mathrm{THF}-\mathrm{d}_{8}, 161 \mathrm{MHz}, 298 \mathrm{~K}$.

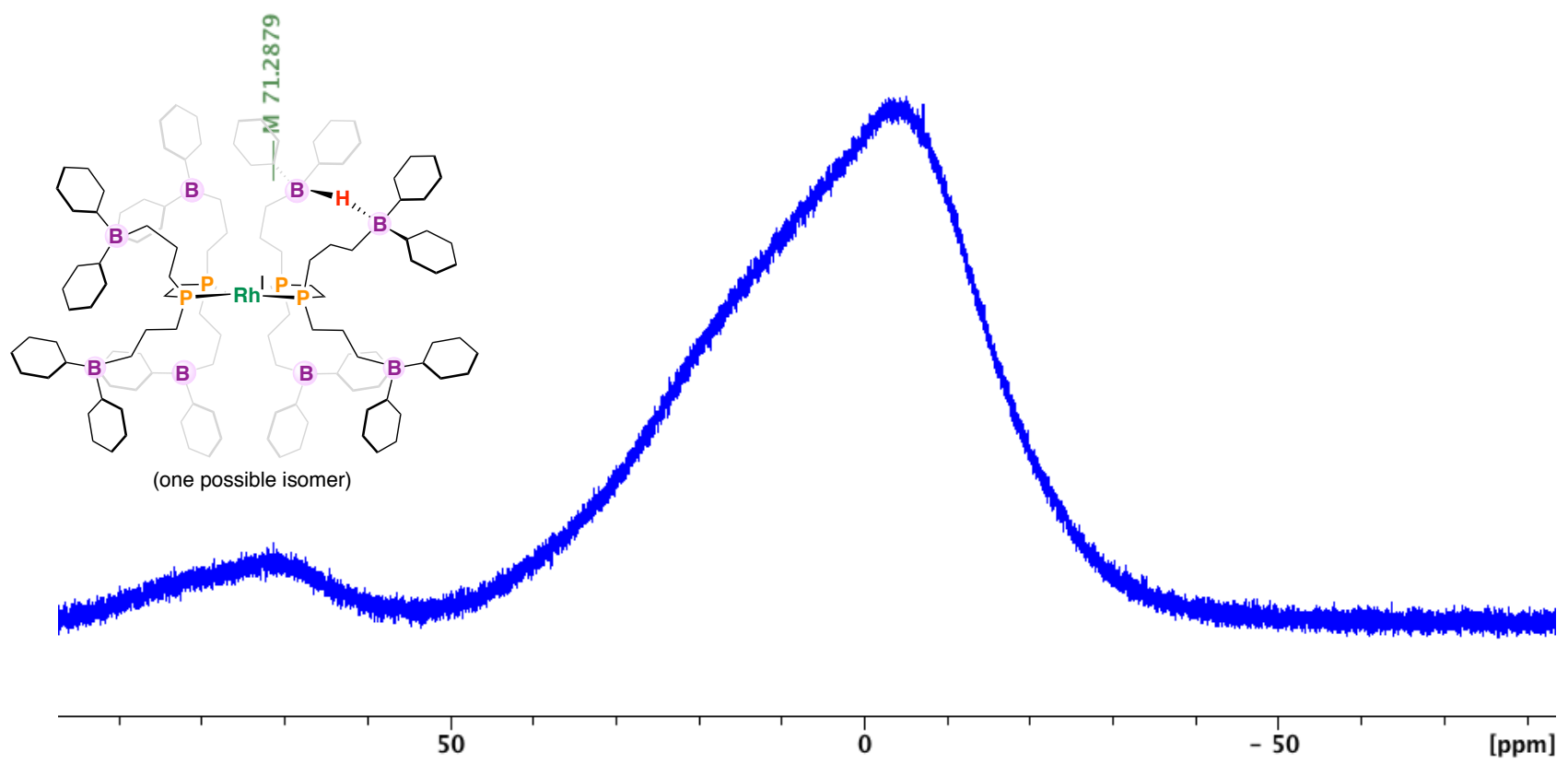

Figure S46. 7, ${ }^{13} \mathrm{C}\left\{{ }^{1} \mathrm{H}\right\}$ NMR, THF- $\mathrm{d}_{8}, 125 \mathrm{MHz}, 298 \mathrm{~K}$ ( ${ }^{*}$ Signals at $\delta \mathrm{c}=14.2,23.3,32.3$ are due to $n$-hexanes).
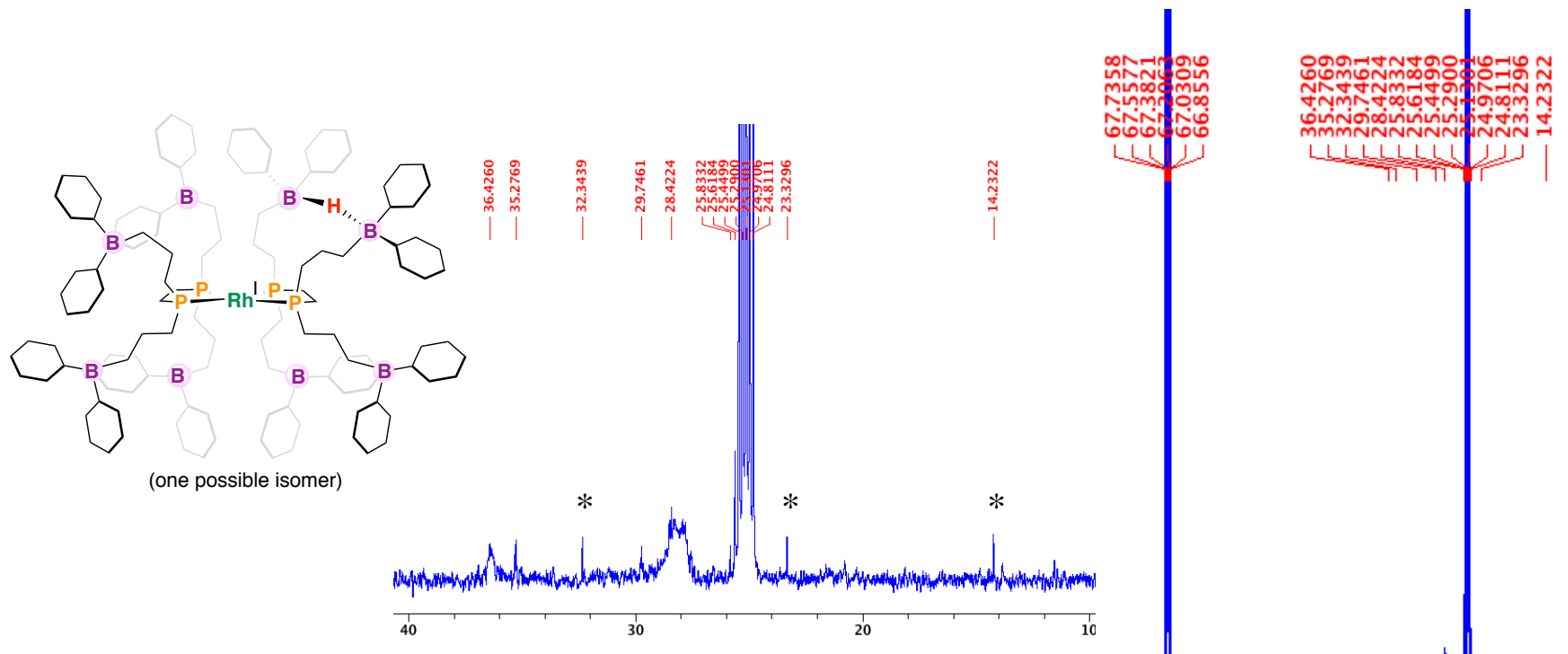

(one possible isomer)

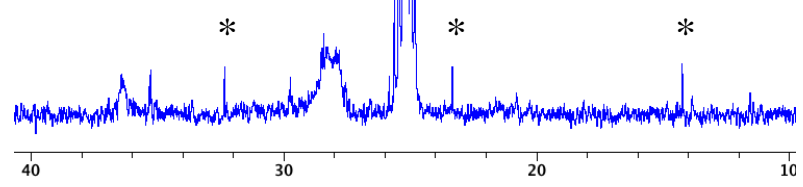


Figure S47. 7, Variable temperature ${ }^{1} \mathrm{H}$ NMR, THF- $\mathrm{d}_{8}, 500 \mathrm{MHz}$. Upfield shifted $\alpha$ borate-protons are highlighted.

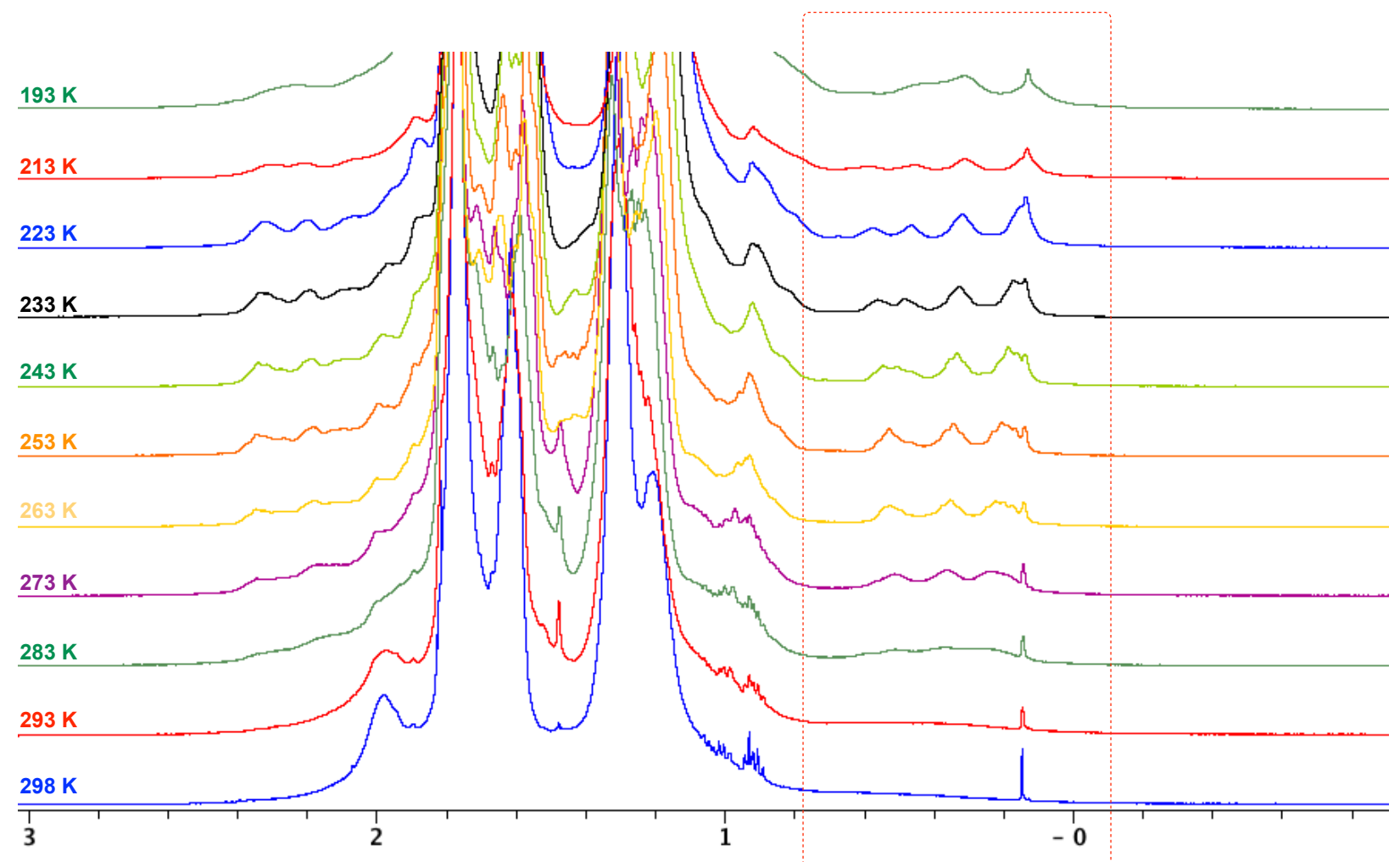

Figure S48. 7, Variable temperature ${ }^{31} \mathrm{P}\left\{{ }^{1} \mathrm{H}\right\} \mathrm{NMR}, \mathrm{THF}-\mathrm{d}_{8}, 203 \mathrm{MHz}$.

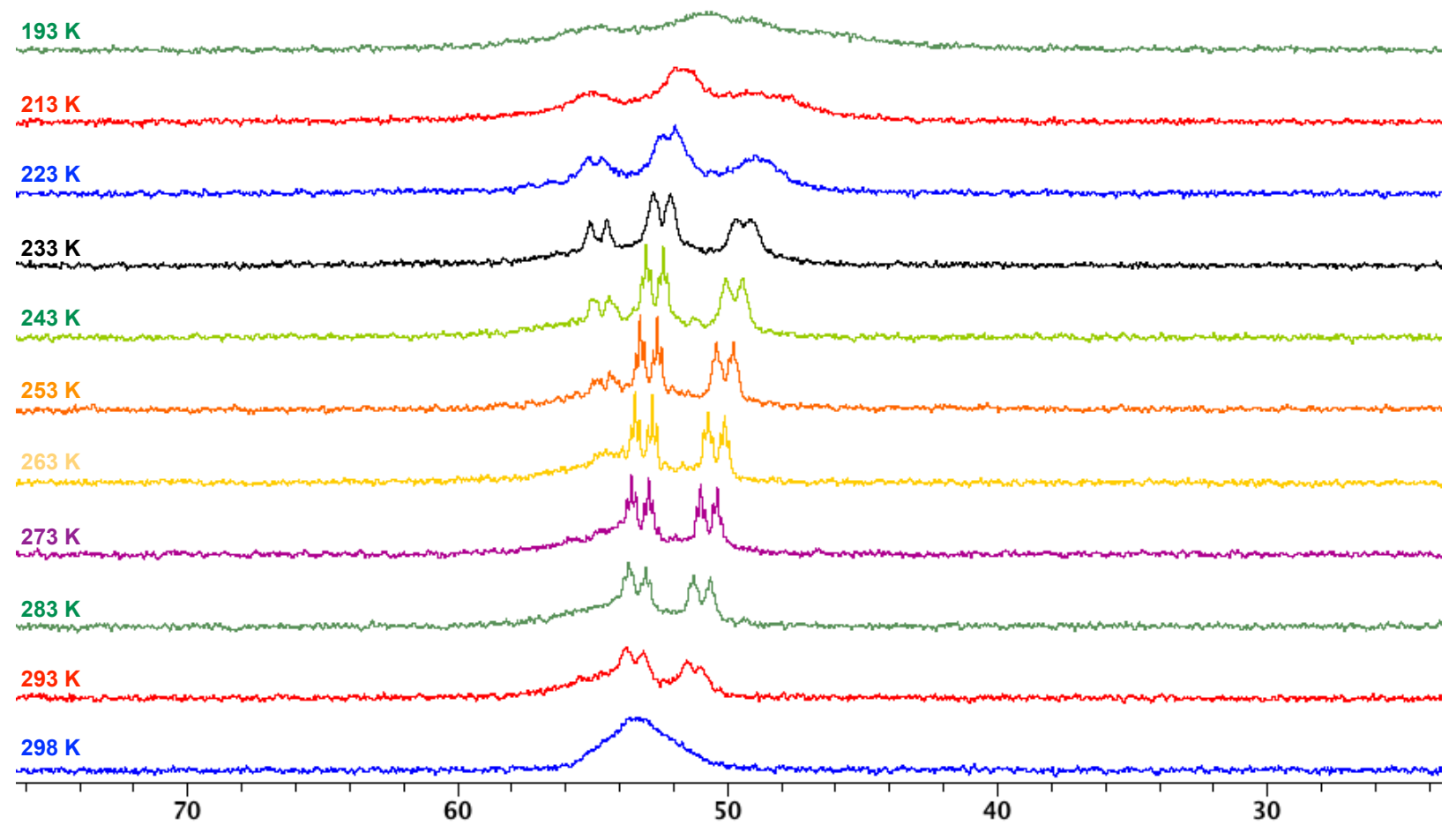


Figure S49. ${ }^{31} \mathrm{P}\left\{{ }^{1} \mathrm{H}\right\} \mathrm{NMR}, \mathrm{THF}-\mathrm{d}_{8}, 203 \mathrm{MHz}, 298 \mathrm{~K}$ for the treatment of $\left[\mathrm{Rh}(\mathrm{d} n \mathrm{ppe})_{2}(\mathrm{H})\right]$ (5) with $\mathrm{BCy}{ }^{n}$ Oct ( 1 equiv. and 8 equiv.), indicating that hydride transfer is dependent on borane concentration.

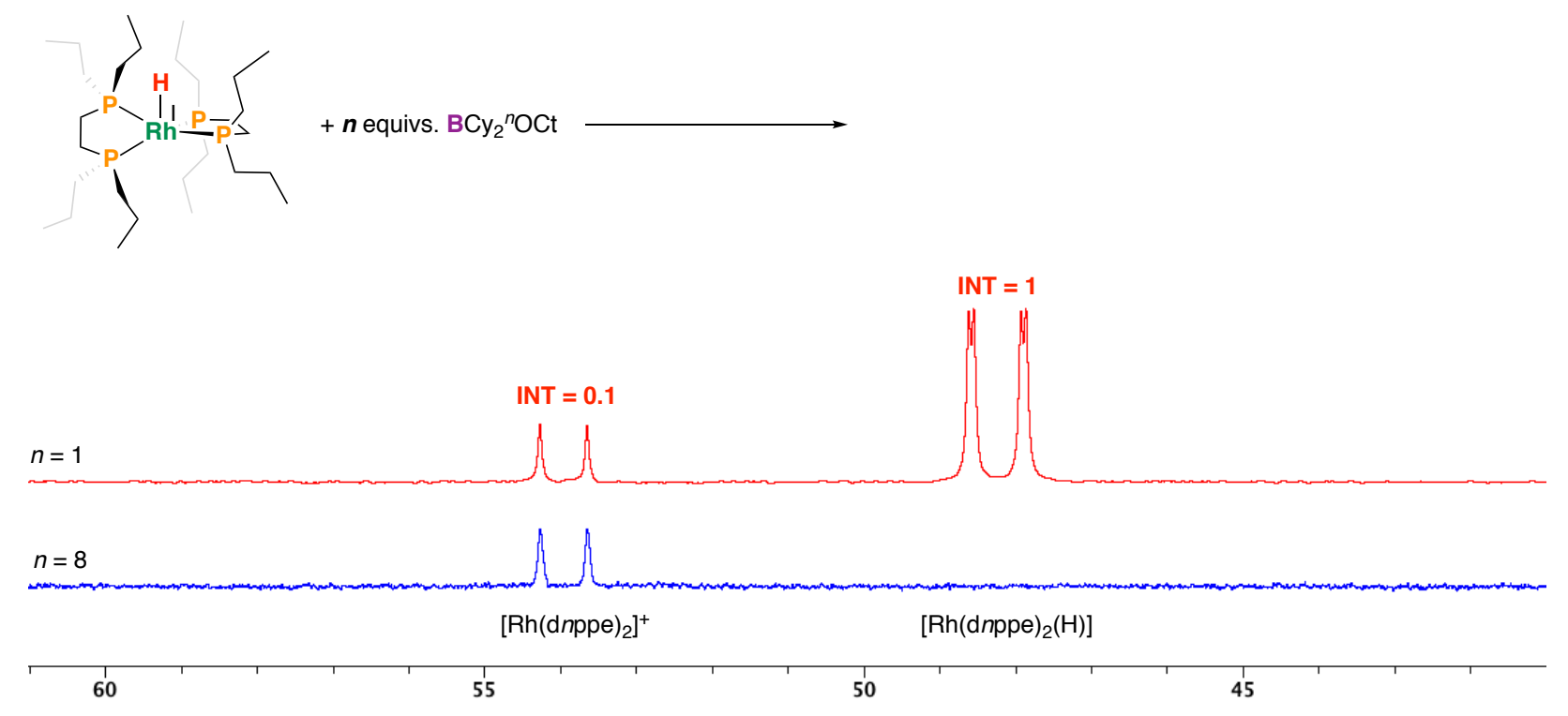

Figure S50. 8, ${ }^{1} \mathrm{H}$ NMR, THF-d $8,500 \mathrm{MHz}, 298 \mathrm{~K}$.

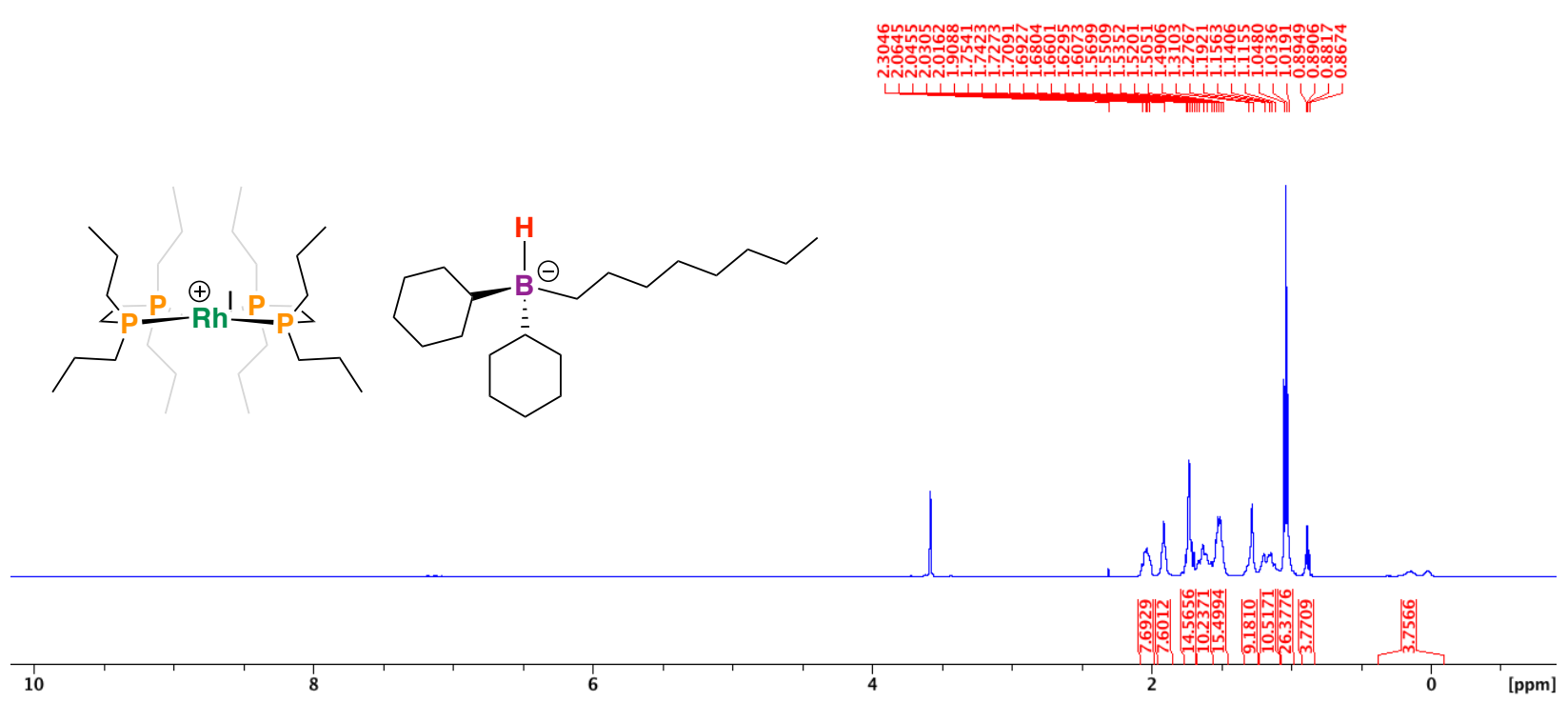


Figure S51. 8, ${ }^{1} \mathrm{H}$ NMR, THF-d $8,500 \mathrm{MHz}, 298 \mathrm{~K}$.

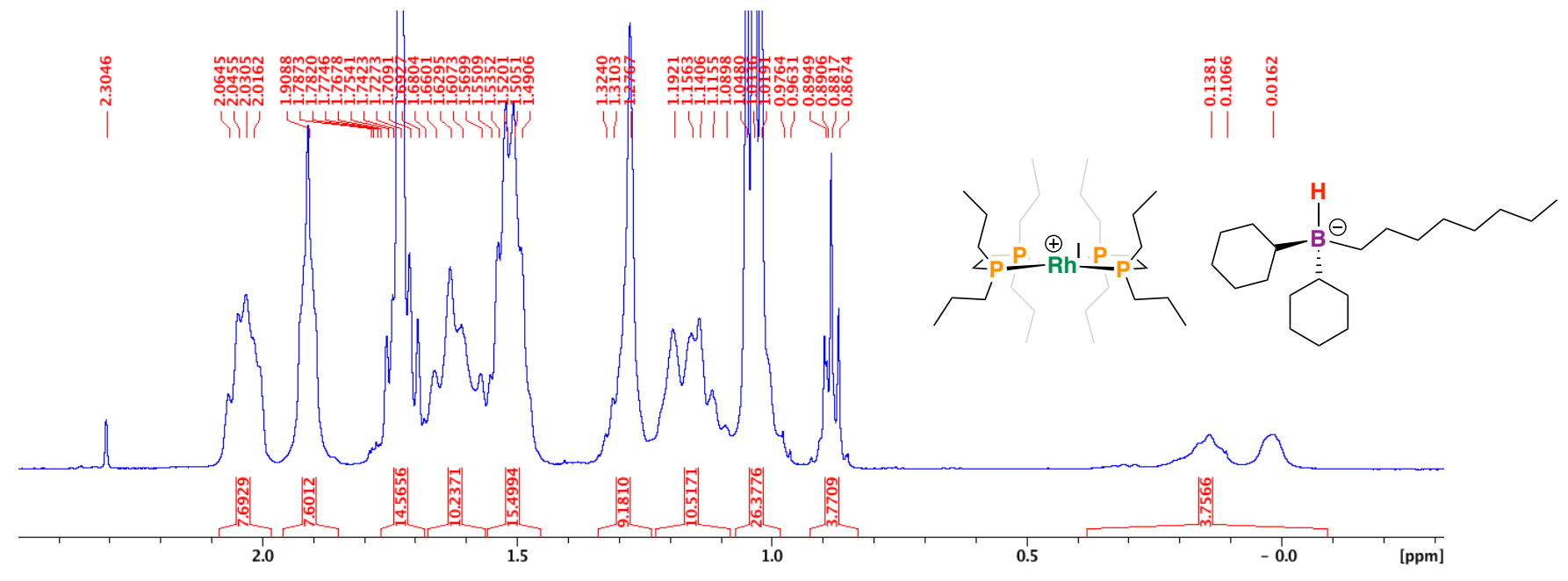

Figure S52. 8, a) ${ }^{1} \mathrm{H}$ NMR, b) ${ }^{1} \mathrm{H}\left\{{ }^{31} \mathrm{P}\right\}$ NMR (o2p = $\left.54 \mathrm{ppm}\right)$, and c) ${ }^{1} \mathrm{H}\left\{{ }^{11} \mathrm{~B}\right\}$ NMR $(\mathrm{o} 2 \mathrm{p}=-$ $4.6 \mathrm{ppm}), \mathrm{THF}-\mathrm{d}_{8}, 500 \mathrm{MHz}, 298 \mathrm{~K}$.

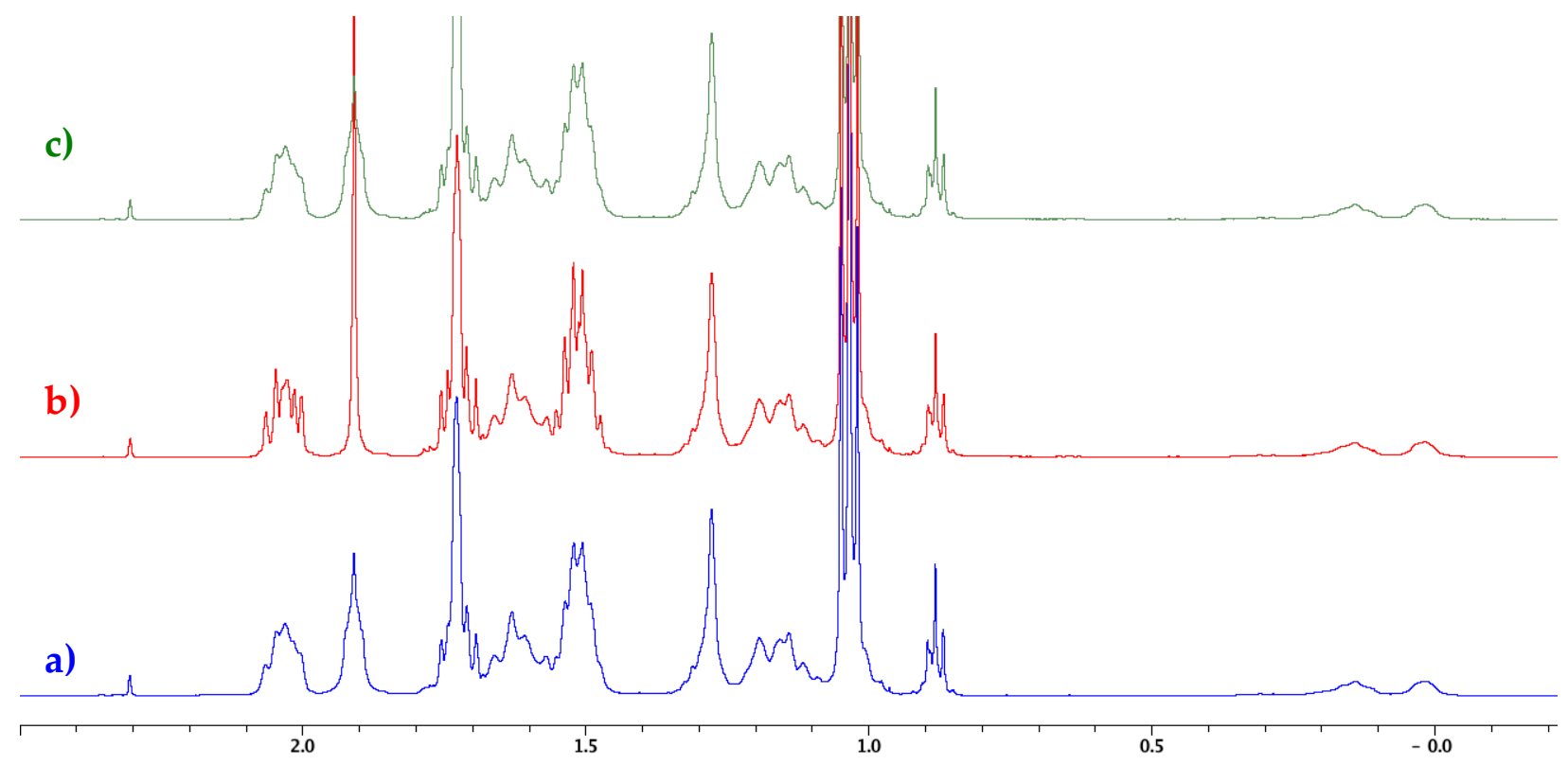


Figure S53. 8, ${ }^{31} \mathrm{P}\left\{{ }^{1} \mathrm{H}\right\} \mathrm{NMR}, \mathrm{THF}-\mathrm{d}_{8}, 203 \mathrm{MHz}, 298 \mathrm{~K}$.

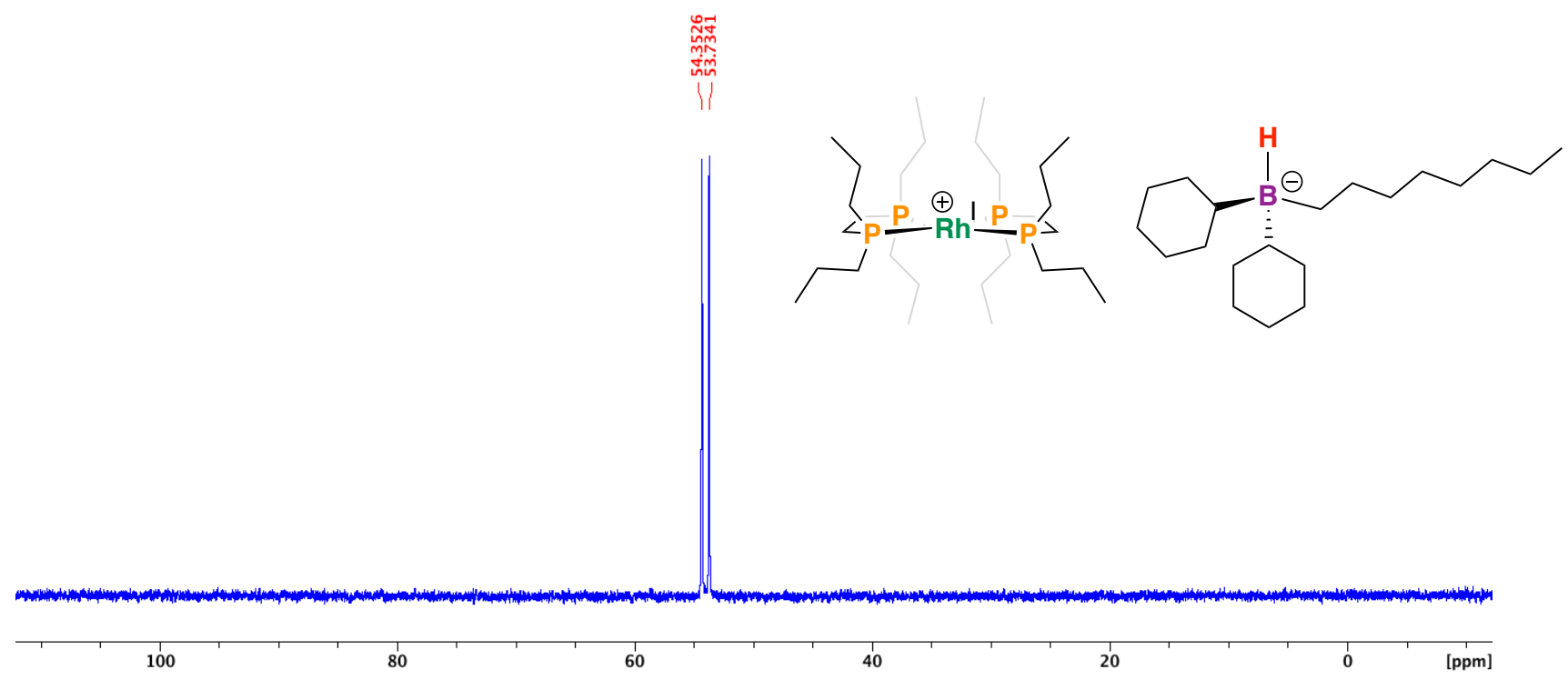

Figure S54. ${ }^{1} \mathrm{H}$ NMR a) reaction of 5 with 2 equivs. $\mathrm{BC} \mathrm{y}_{2}{ }^{\mathrm{n}}$ Oct and $\mathrm{b}$ ) isolated 8 (terminal $\mathrm{BH})$ showing upfield-shifted $\alpha$-borate signals, THF- $\mathrm{d}_{8}, 500 \mathrm{MHz}$. In a) the $\alpha$-CHx groups are shifted more downfield, possibly suggesting a $\mu-\mathrm{B}-\mathrm{H}-\mathrm{B}$ bonding scenario, whereas for $\mathbf{b}$ ) in isolated $\mathbf{8}$, these signals move upfield (terminal borohydride) due to removal of excess $\mathrm{BCy} 2^{\mathrm{n}}$ Oct.

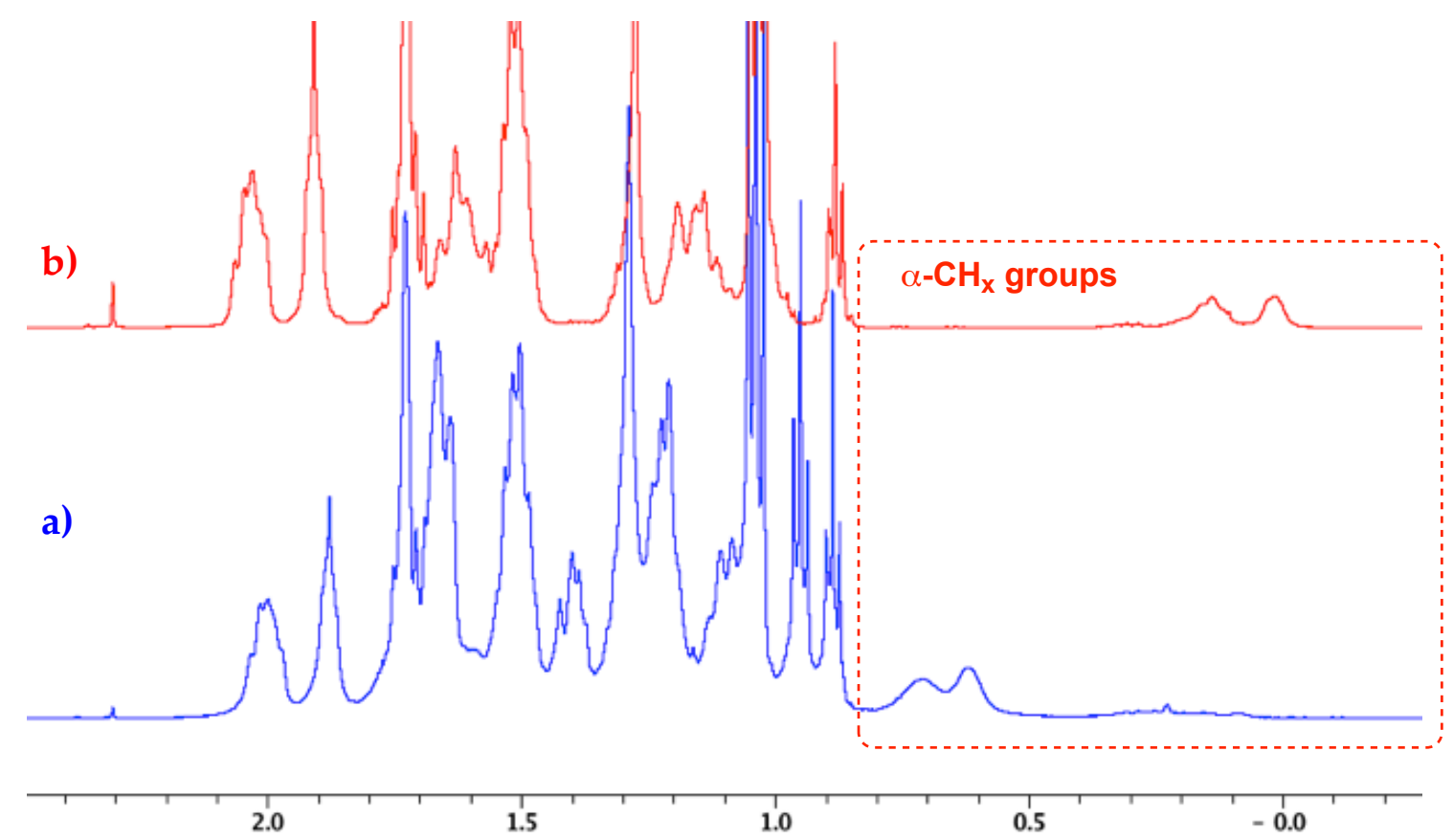


Figure S55. 8, ${ }^{11} \mathrm{~B}\left\{{ }^{1} \mathrm{H}\right\}$ NMR a) isolated 8 (terminal $\left.\mathrm{BH}\right)$ and b) reaction of 5 with 2 equivs. $\mathrm{BCy}_{2}{ }^{\mathrm{n}} \mathrm{Oct}, \mathrm{THF}-\mathrm{d}_{8}, 161 \mathrm{MHz}, 298 \mathrm{~K}$. In a) a terminal borohydride is consistent with $\delta_{в}=-4.5$ ppm.

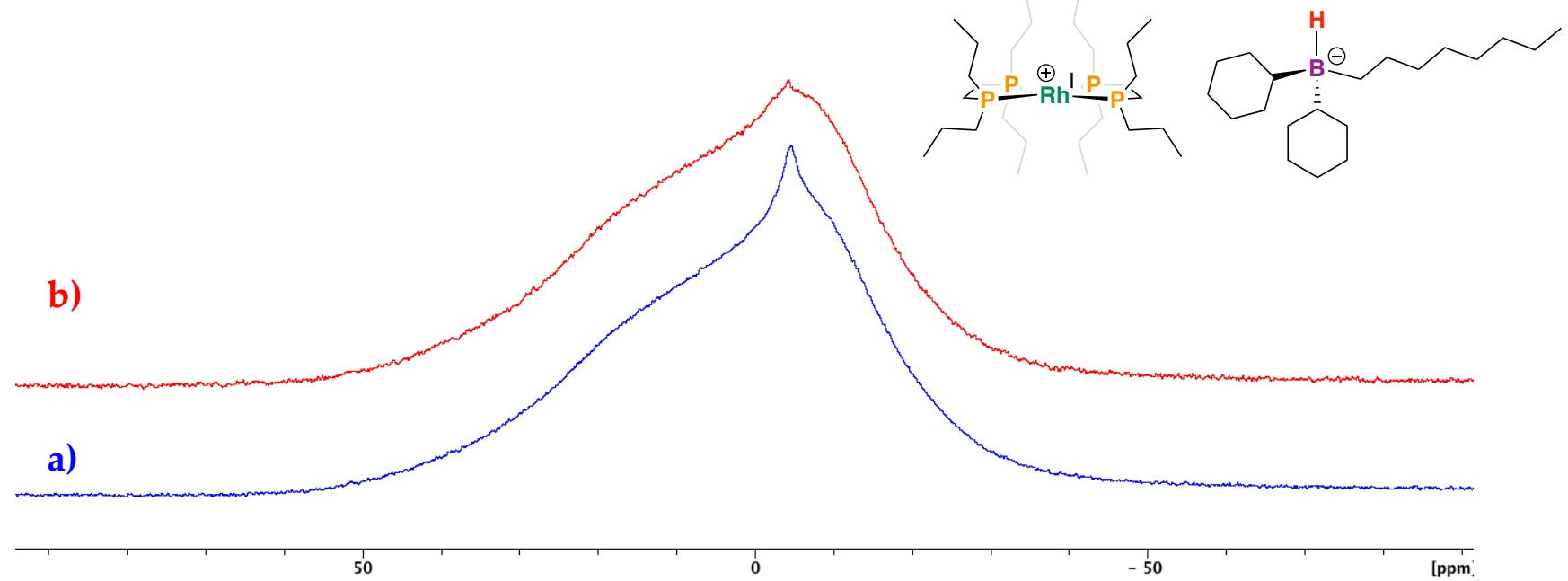

Figure S56. ${ }^{1} \mathrm{H}$ NMR a) $8(298 \mathrm{~K})$ and b) 7 (243 K) showing upfield-shifted $\alpha$-borate signals, THF- $\mathrm{d}_{8}, 500 \mathrm{MHz}$.

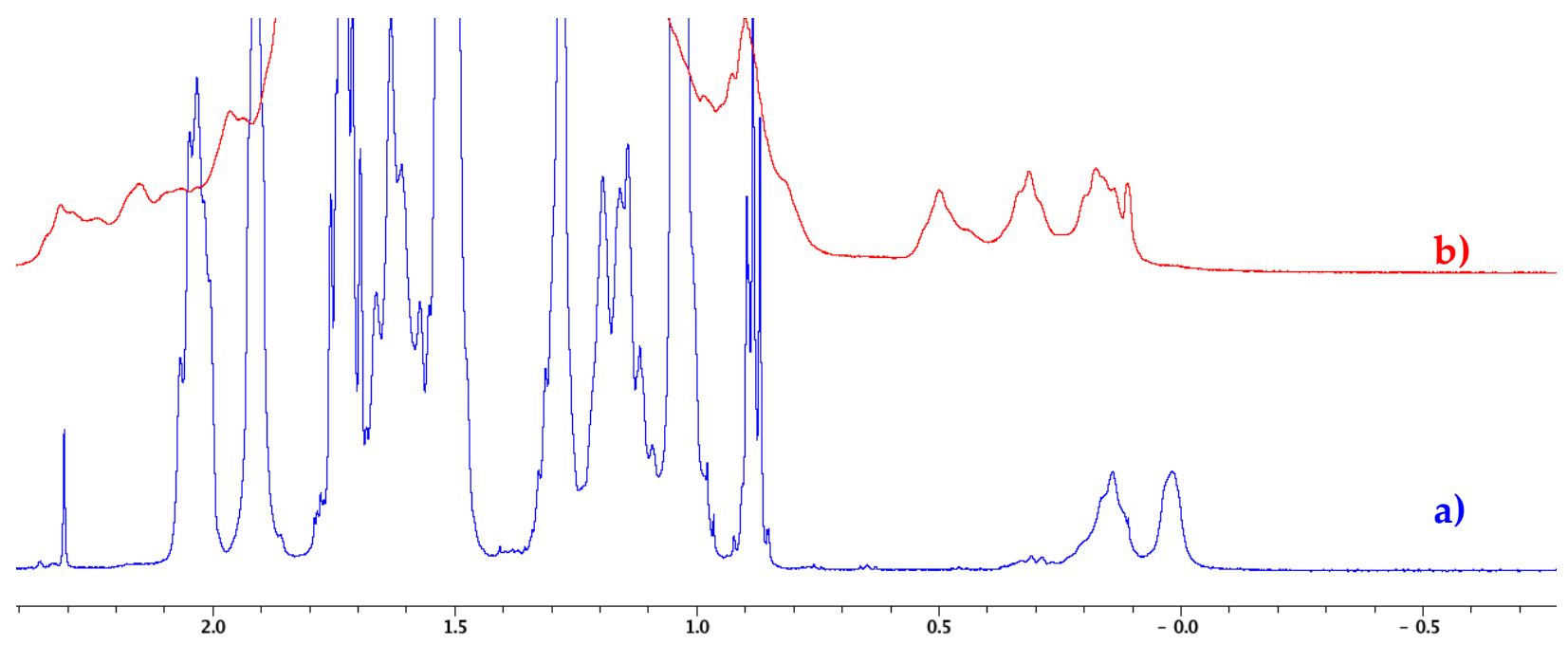


Figure S57. ATR FT-IR, thin-film under $\mathrm{N}_{2}, 298 \mathrm{~K}$ for a) Complex 5, b) K[BEt $\left.3 \mathrm{H}\right](1.0 \mathrm{M}$ in THF), c) Reaction mixture of complex $[3]^{+}+$complex 5. For complex 5, a band at $v$ $1875 \mathrm{~cm}^{-1}$ is comparable to that for $\left[\mathrm{Rh}(\mathrm{dmpe})_{2} \mathrm{H}\right]\left(v=1852 \mathrm{~cm}^{-1}\right){ }^{2}$ while bands for $\mathrm{K}\left[\mathrm{BEt} \mathrm{t}_{3} \mathrm{H}\right]\left(\mathrm{v}=2014 / 1951 \mathrm{~cm}^{-1}\right)$ are comparable to those for free $\mathrm{K}\left[\mathrm{BEt} \mathrm{t}_{3} \mathrm{H}\right]\left(v=2020 \mathrm{~cm}^{-1}\right.$, br) and for $\mathrm{K}[\mathrm{BEt} 3 \mathrm{H}]$ with some BEts present $\left(v=1940 \mathrm{~cm}^{-1}, \mathrm{v}\right.$ br for K[BEt $\left.3 \mathrm{H}\right]+1$ equiv. $\left.\mathrm{BEt}_{3}\right) .^{3}$ No signal $(\mathrm{B}-\mathrm{H}$ nor $\mathrm{Rh}-\mathrm{H})$ is observed for $\mathbf{7}$ formed via treatment of complex $[3]^{+}$with 1 equiv. complex 5.

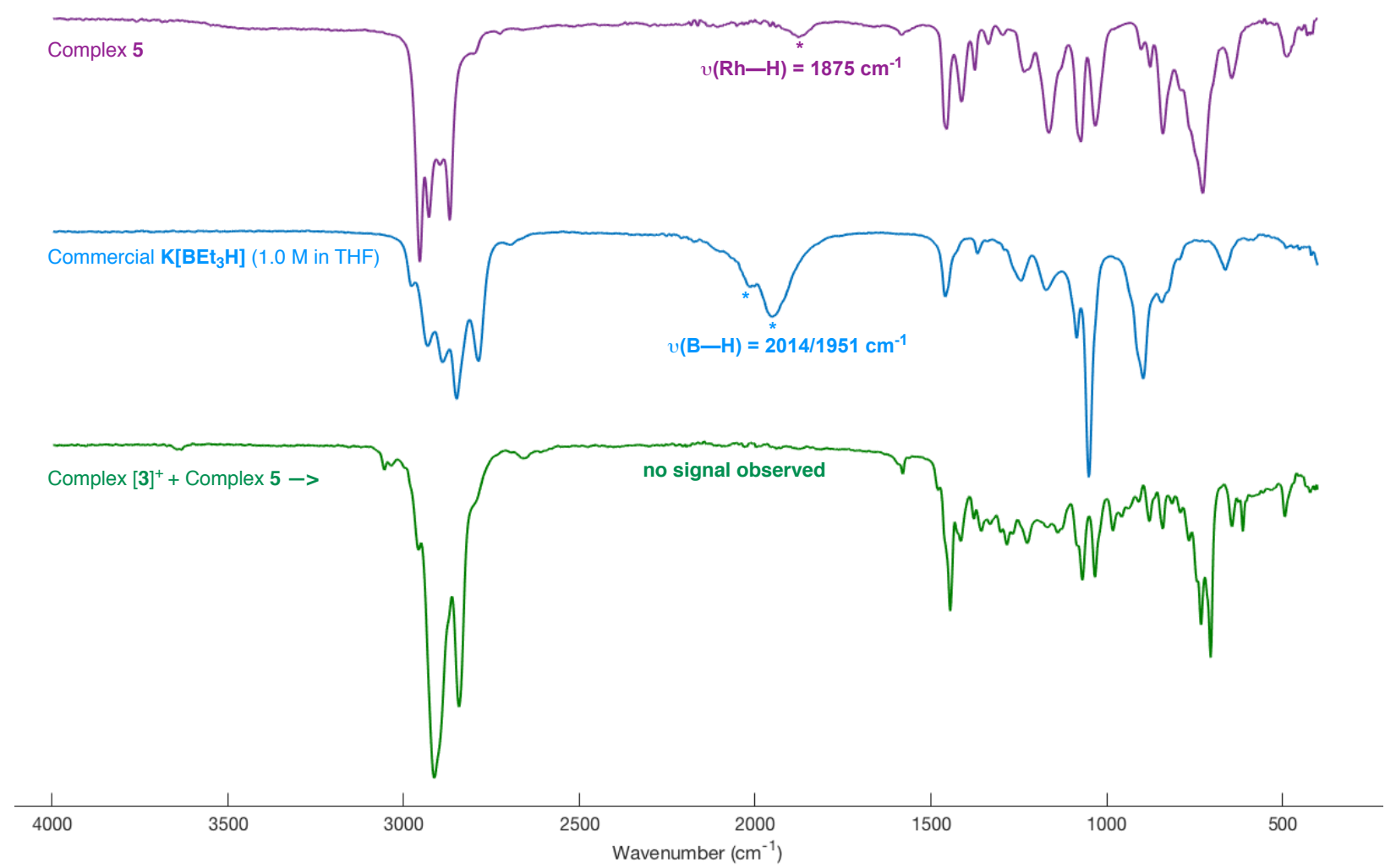

${ }^{2}$ D. Zargarian, P. Chow, N. J. Taylor, and T. B. Marder, J. Chem. Soc. Chem. Commun. 1989, 540. 
Figure S58. Comparison of a) treatment of $\left[\mathrm{Rh}(\operatorname{dnppe})_{2}(\mathrm{H})\right](5)$ with $\left[\mathrm{BNAcP} \mathrm{PF}_{6}, \mathrm{~b}\right)$ treatment of $\left[\mathrm{Rh}\left(\mathrm{P}_{2} \mathrm{~B}^{\mathrm{C}_{4}}-\mu-\mathrm{B}-\mathrm{H}-\mathrm{B}\right)\left(\mathrm{P}_{2} \mathrm{~B}^{\mathrm{Cy}_{4}}\right)\right]$ (7) with $[\mathrm{BNAcP}] \mathrm{PF}_{6},{ }^{1} \mathrm{H}$ NMR, THF- $\mathrm{d}_{8}, 500$ $\mathrm{MHz}, 298 \mathrm{~K}$. In the case of $\mathbf{b}$ ), both bound and unbound 1,4-[BNAcP]H are observed in a ratio of 1.0:0.1, where bound corresponds to suggested interaction of the carbonyl lone pair with a $\mathrm{BR}_{3}$ unit present on the ligand, rendering the $\mathrm{CH}_{2}$ groups $\left(3^{\prime} / 3^{\prime \prime}\right)$ diastereotopic. In the case of a), only unbound 1,4-[BNAcP]H is observed.

Bound: ${ }^{1} \mathrm{H}$ NMR data (500 MHZ, THF-d8, $298 \mathrm{~K}$, selected signals): $\delta=6.02$ (d, ${ }^{3} \mathrm{H}, \mathrm{H}=7.8$ $\mathrm{Hz}, 1 \mathrm{H}), 5.00\left(\mathrm{dd},{ }^{3} \mathrm{H}_{\mathrm{H}, \mathrm{H}}=7.8 \mathrm{~Hz},{ }^{3} J_{\mathrm{H}, \mathrm{H}}=4.9 \mathrm{~Hz}, 1 \mathrm{H}\right), 4.53(\mathrm{~s}, 2 \mathrm{H}), 3.95(\mathrm{~m}, 1 \mathrm{H}), 3.23(\mathrm{dd}$, $\left.{ }^{2} J_{\mathrm{H}, \mathrm{H}}=13.9 \mathrm{~Hz},{ }^{3} \mathrm{JH}_{\mathrm{H}, \mathrm{H}}=4.6 \mathrm{~Hz}, 1 \mathrm{H}\right), 3.23\left(\mathrm{dd},{ }^{2} \mathrm{~J}_{\mathrm{H}, \mathrm{H}}=13.9 \mathrm{~Hz},{ }^{3} \mathrm{JH}_{\mathrm{H}, \mathrm{H}}=8 \mathrm{~Hz}, 1 \mathrm{H}\right), 2.13(\mathrm{~s}, 3 \mathrm{H})$.

Free: ${ }^{1} \mathrm{H}$ NMR data (500 MHZ, THF-d8, $298 \mathrm{~K}$, selected signals): $\delta=5.81$ (d, ${ }^{3} \mathrm{JH}_{\mathrm{H}, \mathrm{H}}=7.8$ $\mathrm{Hz}, 1 \mathrm{H}), 4.80\left(\mathrm{dt},{ }^{3} \mathrm{H}_{\mathrm{H}, \mathrm{H}}=8.0 \mathrm{~Hz},{ }^{3} \mathrm{H}_{\mathrm{H}, \mathrm{H}}=3.4 \mathrm{~Hz}, 1 \mathrm{H}\right), 4.40(\mathrm{~s}, 2 \mathrm{H}), 3.98(\mathrm{~m}, 1 \mathrm{H}), 2.98(\mathrm{~m}, 2 \mathrm{H})$, $2.05(\mathrm{~s}, 3 \mathrm{H})$.
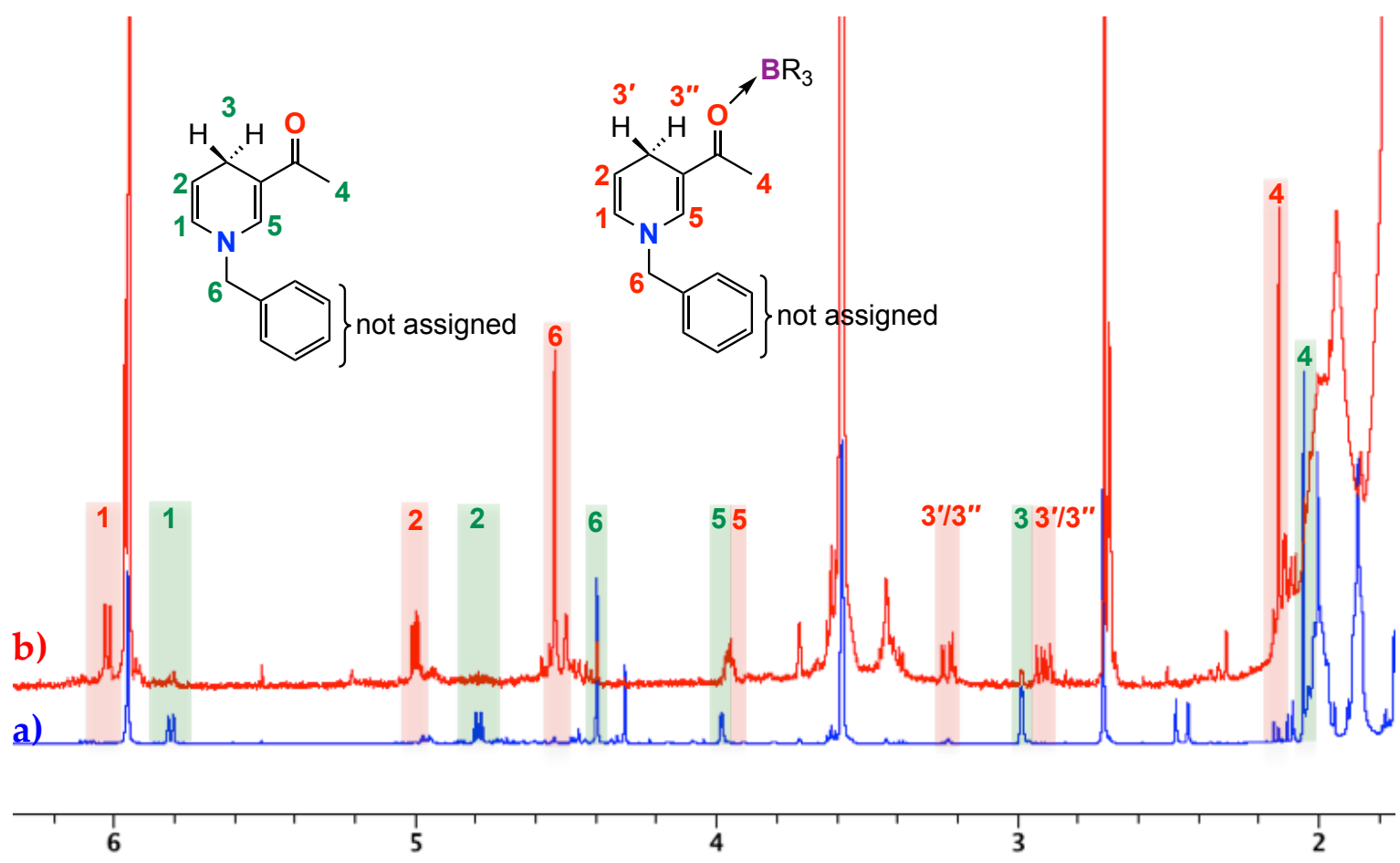


\section{Crystallographic details:}

All crystals were mounted on a mitegen loop. Measurements were made using graphitemonochromated $\mathrm{Cu} \mathrm{K} \alpha(\lambda=1.54178 \AA)$ radiation on a Bruker D8 Venture diffractometer. Structures were solved by direct methods ${ }^{5}$ and refined by full-matrix least-squares procedures on F2 (SHELXL-2013) $)^{3}$ using the OLEX2 interface. ${ }^{4}$ Hydrogen atoms were placed in calculated positions. Non-hydrogen atoms were refined anisotropically.

[1]BPh4: Application of RIGU improved data statistics.

[2]BPh4: One of the $\mathrm{P}-\mathrm{CH}_{2}-\mathrm{CH}_{2}-\mathrm{CH}_{3}$ arms was disordered over two positions and was modeled using two PARTS [50/50]. Application of RIGU improved data statistics. ISOR was used to restrain the ADP of $\mathrm{C} 12$.

CCDC 2069807-2069808 contain the supplementary crystallographic data for this paper. These data can be obtained free of charge from The Cambridge Crystallographic Data Centre via www.ccdc.cam.ac.uk/data_request/cif.

${ }^{3}$ G.M. Sheldrick, Acta Cryst. 2008, A64, 112.

${ }^{4}$ O.V. Dolomanov, L.J. Bourhis, R.J. Gildea, J.A.K. Howard and H.J. Puschmmann, Appl. Cryst. 2009, 42, 339. 
Table S2. Crystallographic data for [1] $\mathrm{BPh}_{4}$ and [2]BPh.

Compound

Empirical formula

Formula weight

Temperature/K

Crystal system

Space group

$\mathrm{a} / \AA$

$\mathrm{b} / \AA$

c/ $\AA$

$\mathrm{a} /{ }^{\circ}$

$\mathrm{b} /{ }^{\circ}$

$\mathrm{g} /{ }^{\circ}$

$\mathrm{V} / \AA^{3}$

$\mathrm{Z}$

$\mathrm{r} / \mathrm{g} / \mathrm{cm}^{-3}$

$\mathrm{m} / \mathrm{mm}^{-1}$

$F(000)$

Crystal size $/ \mathrm{mm}^{3}$

Radiation

$2 \theta$ range for data collection/ ${ }^{\circ}$

Index ranges

Reflections Collected

Independent reflections

Data/restraints/parameters

Goodness-of-fit on $F^{2}$

$\mathrm{R}[\mathrm{I}>=2 \theta(\mathrm{I})](\mathrm{R} 1, \mathrm{wR} 2)$

$\mathrm{R}$ (all data) (R1, wR2)

Largest diff. peak/hole / $\left(\mathrm{e} \AA^{-3}\right)$
[1]BPh4

$\mathrm{C}_{52} \mathrm{H}_{68} \mathrm{BP}_{4} \mathrm{Rh}$

930.66

170(2)

Triclinic

P-1

13.0063(10)

18.0209(14)

22.1147(16)

$76.629(2)$

$87.020(2)$

84.972(3)

$5020.8(7)$

4

1.231

4.189

1960.0

$$
0.20 \times 0.18 \times 0.15
$$

$\mathrm{CuK} \alpha(\lambda=1.54178)$

4.108 to 72.95

$$
-10 \leq \mathrm{h} \leq 10,-13 \leq \mathrm{k} \leq 13,-17 \leq
$$$$
1 \leq 17
$$

57035

$4796\left[R_{\text {int }}=0.0592, R_{\text {sigma }}=\right.$

0.0292]

4796/912/1045

1.094

$\mathrm{R}_{1}=0.0347, \mathrm{wR}_{2}=0.0697$

$\mathrm{R}_{1}=0.0451, \mathrm{wR}_{2}=0.0737$

$0.78 /-0.34$
[2]BPh4

$\mathrm{C}_{52} \mathrm{H}_{84} \mathrm{BP}_{4} \mathrm{Rh}$

946.79

$170(2)$

Monoclinic

$\mathrm{C} 2 / \mathrm{c}$

24.4054(7)

9.2858(3)

22.5491(7)

90

93.517(2)

90

5100.5(3)

4

1.233

4.124

2024.0

$0.2 \times 0.15 \times 0.12$

$\mathrm{CuK} \alpha(\lambda=1.54178)$

7.258 to 99.668

$-24 \leq \mathrm{h} \leq 24,-9 \leq \mathrm{k} \leq 9,-22 \leq 1 \leq$

$$
\begin{gathered}
22 \\
26094 \\
2599\left[R_{\text {int }}=0.0564, R_{\text {sigma }}=\right. \\
0.0316] \\
2599 / 243 / 297 \\
1.111 \\
\mathrm{R}_{1}=0.0343, \mathrm{wR}_{2}=0.0646 \\
\mathrm{R}_{1}=0.0488, \mathrm{wR}_{2}=0.0694 \\
0.23 /-0.26
\end{gathered}
$$

$\mathrm{R} 1=\Sigma|| \mathrm{F}_{\mathrm{o}}|-| \mathrm{F}_{\mathrm{c}}|| / \Sigma\left|\mathrm{F}_{\mathrm{o}}\right| ; \mathrm{wR} 2=\left[\Sigma\left(\mathrm{w}\left(\mathrm{F}_{\mathrm{o}}{ }^{2}-\mathrm{F}_{\mathrm{c}}\right)^{2}\right) / \Sigma \mathrm{w}\left(\mathrm{F}_{\mathrm{o}}\right)^{2}\right]^{1 / 2}$ 


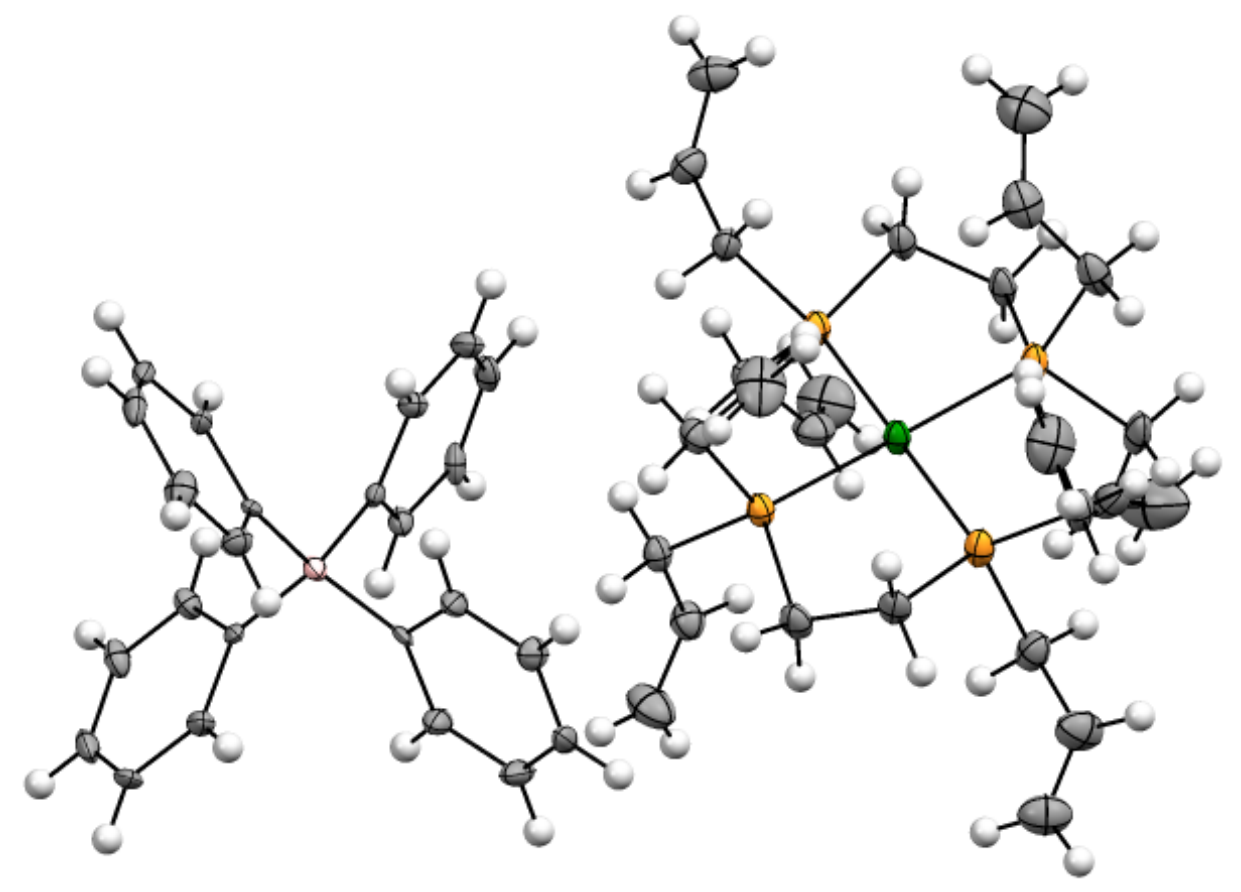

Figure S59. Mercury depiction of $[1] \mathrm{BPh}_{4}$ (elipsoids at 20\% probability).

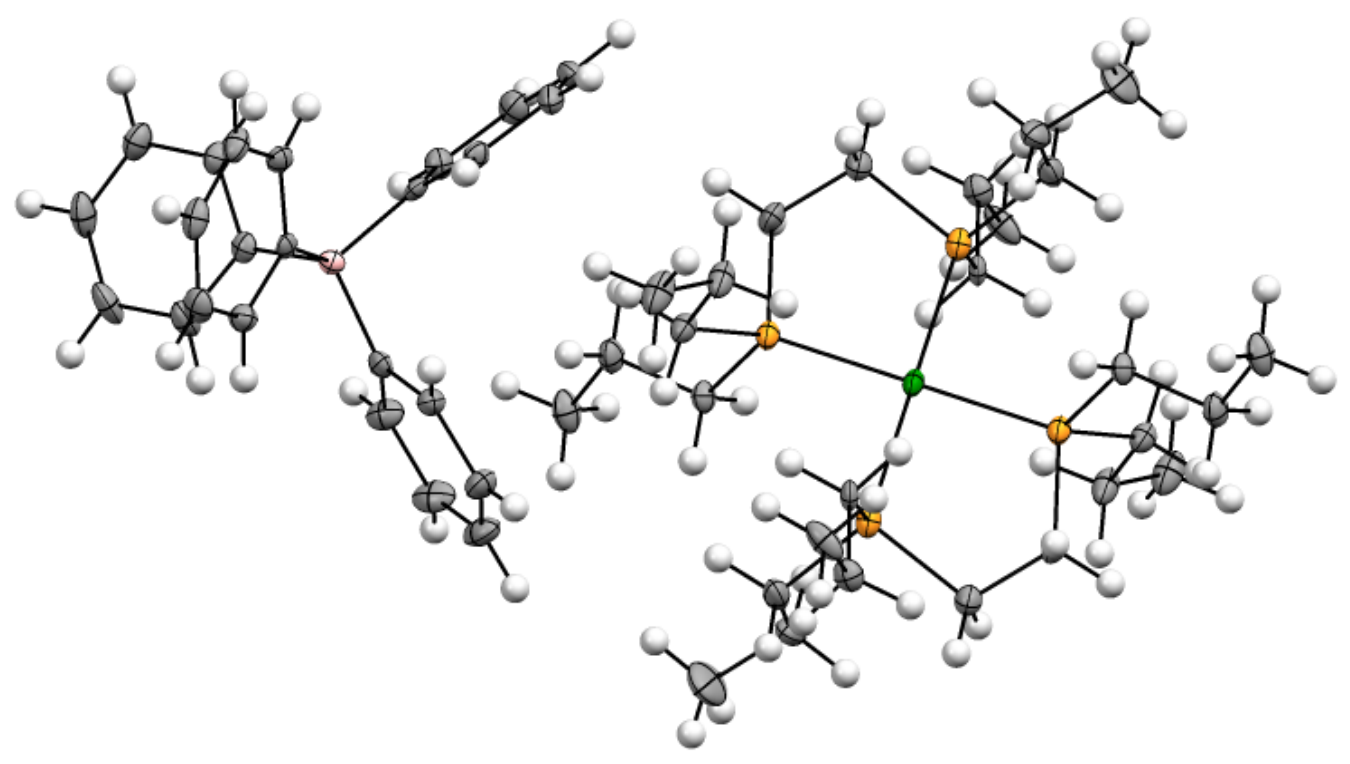

Figure S60. Mercury depiction of $[1] \mathrm{BPh}_{4}$ (elipsoids at 20\% probability). 


\section{Computational details:}

All calculations were performed using version 4.2.1 of the ORCA computational package $^{5}$ and were run on the Cedar cluster at Simon Fraser University, maintained by Compute Canada. All geometry optimizations and frequency calculations were performed at the BP86-D3(BJ)/def2-TZVP' ${ }^{6}$ level of theory using tight optimization (TIGHTOPT) methods. The RI approximation was used to enhance computational efficiency, along with the auxiliary basis $d e f 2 / J^{7}$. Convergence criteria were met using Grid4 and FinalGrid6 integral grid sizes. Frequency calculations were performed to confirm that each optimized geometry was a true minimum indicated by the absence of imaginary frequencies. Grid sizes of Grid4 FinalGrid6 were used for geometry optimizations. Thermochemical corrections for the electronic energies were obtained from the frequency calculations. An effective-core-potential (ECP) ${ }^{8}$ was automatically generated by ORCA on Rh. An .xyz file containing pertinent geometries can be found in the supporting information. Accurate electronic energies were calculated using the "gold standard" of quantum chemistry $(\mathrm{CCSD}(\mathrm{T}))$ at the DLPNO-CCSD(T)/def2-TZVP level of theory. NormalPNO TIGHTSCF were used. The RIJCOSX approximation was used to enhance computational efficiency during the DLPNO-CCSD(T)/def2-TZVP jobs. As such, a def2/ $]^{7}$ auxiliary basis set was used. As well, a def2-TZVP/ $C^{9}$ auxiliary basis set was used.

${ }^{11} \mathrm{~B}$ NMR chemical shifts were calculated with reference to $\mathrm{BF}_{3}-\mathrm{OEt}_{2}$ at the BP86D3(BJ)/def2-TZVP level of theory. A universal solvation model (SMD) of tetrahydrofuran (THF) was used for all ${ }^{11} \mathrm{~B}$ NMR chemical shifts. Grid6 NoFinalGrid integral grid sizes were employed. An auxiliary basis set of $d e f 2 / J K^{10}$ was employed as well. ORCA v. 4.2.1 employs the GIAO method for calculating NMR chemical shifts.

$$
\delta=\delta_{\text {ref }}-\delta_{\text {targetmol }}
$$

\footnotetext{
${ }^{5}$ F. Neese, "Software update: the ORCA program system, version 4.0" WIREs Comput. Mol. Sci. 2017, e1327. doi: $10.1002 /$ wcms.1327

6 a) S. Grimme, S. Ehrlich, L. Goerigk. J. Comput. Chem. 2011, 32, 1456; b) S. Grimme, J. Antony, S. Ehrlich, H. Krieg. J. Chem. Phys. 2010, 132, 154104; c) F. Weigend, R. Ahlrichs. Phys. Chem. Chem. Phys. 2005, 7, 3297.

7 F. Weigend. Phys. Chem. Chem. Phys. 2006, 8, 1057

${ }^{8}$ D. Andrae, U. Haeussermann, M. Dolg, H. Stoll, H. Preuss. Theor. Chim. Acta 1990, 77, 123.

9 A. Hellweg, C. Hattig, S. Hofener, W. Klopper. Theor. Chem. Acc. 2007, 117, 587.

${ }^{10}$ F. Weigend. J. Comput. Chem. 2008, 29, 167.
} 
Table S3. $v(\mathrm{E}-\mathrm{H})(\mathrm{E}=\mathrm{Rh}$ or $\mathrm{B})$ calculated at the BP86-D3(BJ)/def2-TZVP level of theory.

$\begin{array}{cc}\text { Compound } & v(\mathrm{E}-\mathrm{H}) / \mathbf{c m}^{-1} \\ {\left[\mathrm{Rh}(\mathrm{H})(\mathrm{d} n \mathrm{ppe})_{2}\right](5)} & 1901(\mathrm{E}=\mathrm{Rh}) \\ 7^{\mathrm{Me}}(\text { Isomer I }) & 1899(\mathrm{E}=\mathrm{Rh}) \\ 7^{\mathrm{Me}}(\text { Isomer II }) & 2107(\mathrm{E}=\mathrm{B}) \\ 7^{\mathrm{Me}}(\text { Isomer III }) & 1976(\mathrm{E}=\mathrm{B}) \\ 7^{\mathrm{Me}}(\text { Isomer IV }) & 1971(\mathrm{E}=\mathrm{B}) \\ 7^{\mathrm{Me}}(\text { Isomer V) } & 1837(\mathrm{E}=\mathrm{B})\end{array}$
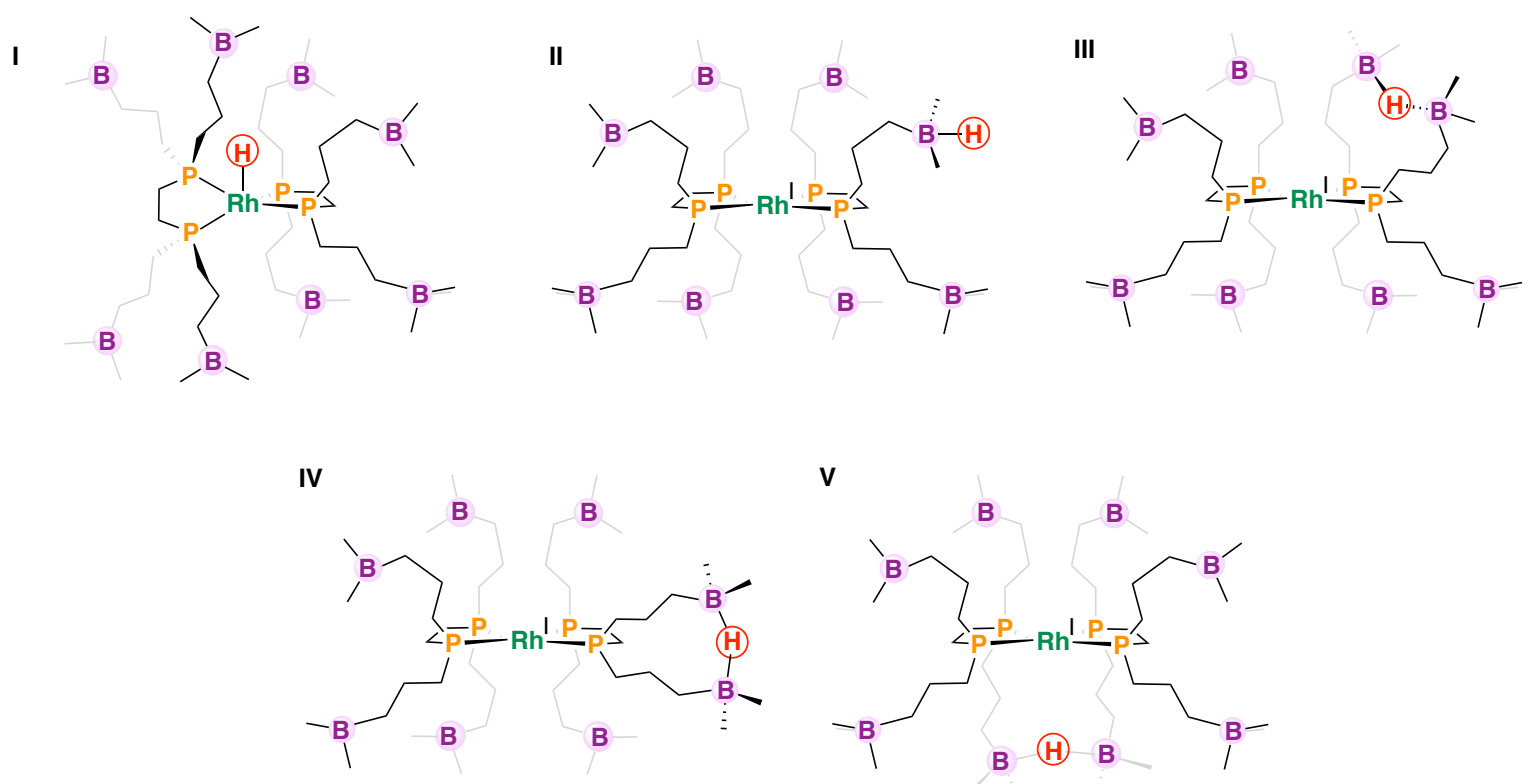

Figure S61 E-H Isomers described in this study. 
Table S4. Calculated ${ }^{11} \mathrm{~B}$ NMR chemical shifts referenced to $\mathrm{BF}_{3}-\mathrm{OEt}_{2}$, calculated at the BP86-D3(BJ)/def2-TZVP level of theory with a universal solvation model (SMD) of THF.

\begin{tabular}{|c|c|c|c|}
\hline Compound & $\begin{array}{r}\text { Indivic } \\
\text { sh }\end{array}$ & $\begin{array}{l}\text { nemical } \\
\text { m }\end{array}$ & $\delta B_{\text {iso }} / \mathrm{ppm}$ \\
\hline \multirow{8}{*}[\mathrm{Rh}(\mathrm{P}_{2}\mathrm{B}^{\mathrm{Me}_{4}})_{2}]{$^{+}\left(\left[3^{\mathrm{Me}}\right]^{+}\right)$} & $\mathrm{B}_{\mathrm{A}}$ & 85 & \multirow{8}{*}{85} \\
\hline & $\mathrm{B}_{\mathrm{B}}$ & 85 & \\
\hline & $\mathrm{Bc}$ & 85 & \\
\hline & $\mathrm{B}_{\mathrm{D}}$ & 84 & \\
\hline & $\mathrm{B}_{\mathrm{E}}$ & 84 & \\
\hline & $\mathrm{B}_{\mathrm{F}}$ & 85 & \\
\hline & $\mathrm{B}_{\mathrm{G}}$ & 85 & \\
\hline & $\mathrm{B}_{\mathrm{H}}$ & 85 & \\
\hline \multirow{8}{*}{$7^{\mathrm{Me}}$ (Isomer II) } & $\mathrm{B}_{\mathrm{A}}$ & 86 & \multirow{8}{*}{71} \\
\hline & $\mathrm{B}_{\mathrm{B}}^{*}$ & -24 & \\
\hline & $\mathrm{BC}$ & 85 & \\
\hline & $\mathrm{BD}_{\mathrm{D}}$ & 85 & \\
\hline & $\mathrm{B}_{\mathrm{E}}$ & 84 & \\
\hline & $\mathrm{B}_{\mathrm{F}}$ & 85 & \\
\hline & $\mathrm{B}_{\mathrm{G}}$ & 85 & \\
\hline & $\mathrm{BH}_{\mathrm{H}}$ & 85 & \\
\hline \multirow{5}{*}{$7^{\mathrm{Me}}$ (Isomer III) } & $\mathrm{B}_{\mathrm{A}}$ & 84 & \\
\hline & $\mathrm{BB}^{*}$ & 1 & \\
\hline & $B c^{*}$ & 3 & \\
\hline & $\mathrm{B}_{\mathrm{D}}$ & 84 & \\
\hline & $\mathrm{B}_{\mathrm{E}}$ & 84 & 64 \\
\hline
\end{tabular}




\begin{tabular}{|c|c|c|c|}
\hline & $\mathrm{B}_{\mathrm{F}}$ & 84 & \\
\hline & $\mathrm{BG}_{\mathrm{G}}$ & 84 & \\
\hline & $\mathrm{B}_{\mathrm{H}}$ & 85 & \\
\hline & $\mathrm{B}_{\mathrm{A}}$ & 85 & \\
\hline & $\mathrm{BB}_{\mathrm{B}}^{*}$ & 0 & \\
\hline & $\mathrm{Bc}^{*}$ & 5 & \\
\hline $7^{\mathrm{Me}}$ (Isomer IV) & $\mathrm{BD}$ & 85 & \\
\hline & $\mathrm{B}_{\mathrm{E}}$ & 85 & \\
\hline & $\mathrm{B}_{\mathrm{F}}$ & 85 & \\
\hline & $\mathrm{B}_{\mathrm{G}}$ & 84 & \\
\hline & $\mathrm{B}_{\mathrm{H}}$ & 84 & \\
\hline & $\mathrm{B}_{\mathrm{A}}$ & 86 & \\
\hline & $\mathrm{BB}_{\mathrm{B}}^{*}$ & 4 & \\
\hline & $\mathrm{Bc}^{*}$ & 5 & \\
\hline $7^{\mathrm{Me}}($ Isomer V) & $\mathrm{B}_{\mathrm{D}}$ & 82 & \\
\hline & $\mathrm{B}_{\mathrm{E}}$ & 84 & \\
\hline & $\mathrm{B}_{\mathrm{F}}$ & 84 & \\
\hline & $\mathrm{B}_{\mathrm{G}}$ & 83 & \\
\hline & $\mathrm{B}_{\mathrm{H}}$ & 84 & \\
\hline
\end{tabular}

